\title{
Nucleation and Growth in Nanosystems: Some New Concepts
}

\author{
A. M. Gusak, A. O. Bogatyrev, A. O. Kovalchuk, \\ S. V. Kornienko, Gr. V. Lucenko, Yu. A. Lyashenko, \\ A. S. Shirinyan, and T. V. Zaporoghets \\ B. Khmel'nyts'ky Cherkasy National University, \\ Sub-Faculty of Theoretical Physics, \\ 81 Shevchenko Blvd., \\ UA-18031 Cherkasy, Ukraine
}

Review covers some new concepts in theory and modelling of the initial stages of solid-state reactions in thin films, multilayers, nanoparticles, and bulk nanocrystalline materials. The following topics are included: possibility of oscillatory ordering and nucleation in the sharp concentration gradient; criteria of suppression/growth of stable and metastable phases at the nucleation stage; possible nucleation modes in sharp concentration gradient and their competition; competitive nucleation and decomposition in small volumes; criteria of unambiguous choosing the parameters of discontinuous precipitation based on the balance and maximum production of the entropy; formation of nanostructure under uniaxial compression of single-crystalline alloy.

Огляд містить набір деяких нових концепцій в теорії і моделюванні початкових стадій твердофазних реакцій у тонких плівках, мультишаpax, наночастинках та в об’ємі нанокристалічних матеріалів. Обговорено наступні теми: можливість осциляційного впорядкування і зародкоутворення в полі градієнта концентрації; критерій пригнічення/росту стабільних і метастабільних фаз на стадії зародкоутворення; можливість різних мод зародкоутворення в полі градієнта концентрації та їх конкуренція; конкурентне зародкоутворення і розпад в малих об'ємах; критерій однозначного вибору параметрів коміркового розпаду, що базується на балансі та максимумі виробництва ентропії; формування наноструктур під дією одновісного стискання монокристалічного стопу.

Обзор содержит набор некоторых новых концепций в теории и моделировании начальных стадий твердофазных реакций в тонких пленках, мультислоях, наночастицах и в объеме нанокристаллических материалов. Обсуждаются следующие темы: возможность осцилляционного упорядочения и зародышеобразования в поле градиента концентрации; критерий подавления/роста стабильных и метастабильных фаз на ста- 
дии зародышеобразования; возможность различных мод зародышеобразования в поле градиента концентрации и их конкуренция; конкурентное зародышеобразование и распад в малых объемах; критерий однозначного выбора параметров ячеистого распада, основанный на балансе и максимуме производства энтропии; формирование наноструктур под воздействием одноосного сжатия монокристаллического сплава.

Keywords: concentration gradient, diffusion, nucleation, decomposition, simulation.

(Received 10 February, 2004)

\section{INTRODUCTION (A LITTLE BIT HISTORY)}

Present review is an attempt of summarizing the recent endeavours of modelling and theoretical description of the Big Bang of solid-state reactions (SSR) at the very initial stages including nucleation. Evident nano-trend of materials science makes initial stages of SSR (true nanoprocess) an important issue. Our group at the Cherkasy University treats these problems during last two decades, in co-operation with such wonderful researchers as C. Gurov and A. Nazarov (Moscow), P. Desré and F. Hodaj (Grenoble), F. van Loo and A. Kodentsov (Eindhoven), K. N. Tu (Los Angeles), G. Schmitz (Muenster), V. V. Slyozov and L. N. Paritskaya (Kharkiv). Some ideas and new understanding in this field, which nucleated, grew and even partially ripened during this period, include (1) ordering, nucleation, competition and growth of stable and/or metastable intermediate phases in the nanoscale diffusion zone-phase transformations in sharp, time-dependent concentration gradient, (2) competitive nucleation in the isolated binary nanoparticles or bulk samples with very high density of nucleation sites, (3) flux-driven coarsening in nanosystems, (4) possibility of bulk nanocrystalline alloy formation under pulse loading.

So, how does the 'reactive Big Bang' look like?

Till 80's a diffusion community, occupied mainly with diffusion and reactions in macrospecimens, treated the initial stages of these processes as some exotic problem, basically, as theoretical one. Reason was that initial stage is usually being 'forgotten' by the system (in accordance with basic principles of non-equilibrium thermodynamics and statistical mechanics) when the diffusion zone reaches several micrometers.

In case of interdiffusion with full mutual solubility, the notion 'initial stage' means the period till reaching the parabolic regime both for concentration redistribution and for Kirkendall shift. It includes:

1. Formation of non-equilibrium vacancy distribution due to vacancy flux divergence arising from different mobilities of species, and its relaxation due to vacancy sources/sinks [1-4]. 
2. Possible diffusion-induced recrystallization-formation of new grains of solid solution [5].

3. In case of polycrystalline materials, transition from pure grain boundary diffusion (regime $C$ ) to the Fisher regime $(B)$, and then to parabolic regime $(A)[6]$.

In case of reactive diffusion, 'initial stage' means the period till formation and parabolic growth of all stable intermediate phases. It includes, in principle, the following sub-stages:

1. Nucleation of intermediate phases-formation of the new phase overcritical nuclei (islands) at or in the vicinity of initial contact interface [7].

2. Growth of new phase islands and competition (for space and material) between them till formation of the phase layers with more or less planar geometry. In case of ternary and multicomponent systems, the two-phase layers can as well be formed.

3. Overcoming of interface barriers (if they exist) [1, 8-10] and/or the relaxation of vacancy subsystem.

Practical interest in the initial stages of SSR appeared due to investigation of reactive diffusion in thin films. The usually observed oneby-one (sequential) phase growth meant that the initial stage for some phases might well be simultaneously the final stage. If, for example, intermediate phase $A_{1} B_{2}$ appeared to be suppressed by some reasons during the growth of the phase $A_{2} B_{1}$ till the consumption of pure $B$, this phase will never appear at all. Yet, even for explaining phase suppression, people tried to avoid the consideration of nucleation processes. Instead, the suppression has been explained in terms of interplay of diffusion and interfacial barriers. Most successful theory of this type belongs to U. Goesele and K. N. Tu [11]. Introducing some arbitrary rate constants for fluxes across interfaces, they managed to derive expressions for the critical thicknesses of suppressing phases and delay periods of suppressed phases.

Nucleation issues, in terms of standard nucleation approach, but in application to solid-state reactions, have been discussed by F. d'Heurle in 1986 [7].

Situation became even more intriguing with discovery of the solid state amorphizing reactions (SSAR) $[12,13]$ demonstrating the growth of metastable amorphous layer without any evidence of stable phase formation till the amorphous layer reached certain critical thickness of hundreds of nanometres. It looked like the nucleation and/or growth of stable phases have been suppressed not by just low temperature, but by the growing metastable phase.

Detailed DSC-investigations of solid state reactions in multilayers by the groups of K. Barmak and P. Gas demonstrated [14-16] the possibility of the two heat release maxima for the same phase, which means some kind of two-stage phase formation (the first stage, possi- 
bly, being the lateral growth of new phase islands).

Recently G. Schmitz et al. used an atom-probe tomography (TAP) method to investigate the very initial stage of solid-state reactions [5, 17]. This method, with resolution of a few angstroems, provides us with the 3-dimensional distribution of atoms of the reacting species. These experiments clearly show that usually even the first islands of the first phase to grow do not form immediately after contact.

One more important new nano-field governed by nucleation is the formation and properties of bulk nanocrystalline materials. Simultaneous and fast nucleation in highly imperfect supersaturated solid solutions yields very small supply regions capable to feed new phase embryo at each nucleation and growth site. As an example, the so-called bulk metallic glasses (BMG) $[18,19]$ often devitrify with a very high nucleation rate yielding, in a first crystallization stage, to a dispersion of nanocrystals in the amorphous matrix [20]. Arguing that such a high frequency of nucleation cannot be due to heterogeneous nucleation, Kelton [21] has explained such a phenomenon by enrichment with the crystallizing component in a shell, (of atomic size width), surrounding embryos. During quenching, those frozen subcritical embryos, with their surrounding enriched shell serving as a feeding material, become overcritical and practically stop growing after consuming the shell component.

Thus, indeed, the 'nano-vector' of solid-state reactions makes it more and more important to take the nucleation stage into account.

In 1982, one of the authors (A.G.), jointly with his teacher, late Prof. Cyril Gurov (A. A. Bajkov Institute of Metallurgy, Moscow) presented a simple (even 'naïve', as we see it today) model of the phase competition taking into account the nucleation stage of each phase [22]. Actually, the only concept, which had been taken in [22] from the nucleation theory, was the existence of critical nuclei. They appear due to some miracle called the heterophase fluctuations, which are the stochastic events and cannot be described by some deterministic model. Initial idea was just that each phase cannot start from zero thickness; it should start from some critical size particle (about nanometre). Contrary to standard nucleation theory, the critical nuclei of intermediate phases during reactive diffusion are formed in the strongly inhomogeneous region-interface between other phases. Therefore, from the very beginning they have to let the diffusion fluxes passing through themselves. Evidently, fluxes change abruptly when passing across each new-formed boundary of the newly born nucleus, and thus drive the boundary movement. This picture of interface movement due to flux steps is well known for diffusion couples under the name of Stephan problem and means diffusive interactions between neighbouring phases. Yet, the initial width of each phase is taken to be the critical nucleus size (instead of zero). Peculiarity of initial stage is just the 
possibility that the width of some phase nucleus (distance between left and right boundaries can decrease as well as increase. If it decreases, then the nucleus becomes subcritical and should disappear. Usually it happens if the neighbouring phases have larger diffusivity and comparative thickness. Then these neighbours ('vampires' or 'sharks') will destroy and consume all of the new-forming nuclei, making new phase to be present only virtually -in the form of constantly forming (due to heterophase fluctuations) and vanishing (due to diffusive suppression by the neighbours) embryo.

Simple mathematical scheme was built predicting the sequence of phase formation and incubation periods, provided that one knows the integrated Wagner diffusivities and critical nucleus size for each phase. Simplest example of this scheme is presented in Section 3. This scheme was applied to competition with solid solutions [23], to the phase growth under strong electric current [24], to the reactive diffusion in ternary systems [25], and to the phase competition in reacting powder systems [26, 27]. Applications to electric field case demonstrated that the phase spectrum of reaction zone can be influenced and even controlled by strong enough current density. Large current densities become real due to miniaturization of integrated schemes and introducing of the flip-chip technology [27, 28]. Last results on reactive diffusion in UBM-solder contacts under strong current crowding [29] confirm the mentioned idea: without current or under weak current, the reaction between copper and dilute solution of tin in lead at $150^{\circ} \mathrm{C}$ demonstrates only $\mathrm{Cu}_{3} \mathrm{Sn}_{1}$-phase formation; current density of $j>10^{8} \mathrm{~A} / \mathrm{m}^{2}$ leads to formation and fast growth of $\mathrm{Cu}_{6} \mathrm{Sn}_{5}$ phase.

Later we realized that in our nanve model of the phase competition we had taken the inhomogeneity of nucleation region into account only partially. Namely, we treated the diffusive interactions of the newborn nuclei, but we had not considered the possible change of the nucleation barrier, size and shape, caused by the very fact of sharp concentration gradient. Thus, we had to reconsider the thermodynamics of nucleation in the concentration gradient. The very first version of such a theory was presented at the conference 'Defects and Diffusion' (DD-89) in Russia and first published May 1990 [30] (see also [31]). Main idea was as follows: if, prior to intermediate phase formation, narrow layer of metastable solid solution or amorphous alloy had been formed at the base of initial interface, the sharp concentration gradient inside this layer provides decrease of the total bulk driving force of nucleation, and corresponding increase of nucleation barrier. Nuclei were taken to be spheres, appearing in the strongly inhomogeneous concentration profile of the parent phase, so that local driving force of transformation could change significantly from the left to the right along the diameter of nucleus. This effect appeared to be non-negligible, since usually the intermediate phases have very strong concentration de- 
pendence of the Gibbs energy. Main result was the new size dependence of the Gibbs energy; it contained, in addition to the terms of second order (surface energy, positive) and third order (bulk driving force, negative), the new term proportional to the 5 th power of size and the squared concentration gradient:

$$
\Delta G(R)=\alpha R^{2}-\beta R^{3}+\gamma(\nabla C)^{2} R^{5},
$$

$\gamma$ being positive and proportional to the second derivative of the new phase Gibbs energy over concentration. Expression (1) means that, for large enough gradient $\nabla C>\nabla C^{\text {crit }}$ (typically $\nabla C^{\text {crit }} \propto 10^{8} \mathrm{~m}^{-1}$ ), the dependence $\Delta G(R)$ becomes monotonically increasing (infinitely high nucleation barrier) meaning thermodynamic suppression of nucleation by the very sharp concentration gradients. Thus, according to our model, at the very initial stage of reactive diffusion the nucleation can be suppressed even without diffusive competition, just due to too narrow space region, favourable for transformation.

Independently, similar results were published by P. Desré et al. in 1990,1991 [32, 33]. This approach had been applied to description of solid-state amorphizing reactions [32-35], explaining why the stable intermetallics appear in diffusion zone only after amorphous layer exceeds some critical thickness.

In spite of similar results, models [30,31] and [32, 33] of nucleation in the sharp concentration gradient treated quite different possible mechanisms (nucleation modes). In [30, 31], a polymorphous mode has been suggested according to the following picture. Initial diffusion leads to formation and growth of metastable parent solution with sharp concentration profile. When this profile becomes smooth enough to provide sufficient space for compositions favourable for new intermediate phase, this very phase nucleates just by reconstruction of atomic order, without changing immediately the concentration profile (at 'frozen' diffusion)-polymorphic transformation. In [32, 33] the transversal nucleation mode was suggested bearing in mind the following picture: each thin slice of the new-formed nucleus, perpendicular to direction of concentration gradient, is considered as a result of decomposition in corresponding thin infinite slice of parent solution, leading, of course, to redistribution of atoms among new and old phases. In this transversal mode, the redistribution proceeds within each slice, independently on others.

In Ref. [36], one more mechanism has been suggested (and analyzed in more details in Refs. [37, 38]) - total mixing (longitudinal) nucleation mode, when the redistribution of atoms proceeds during nucleation, but only inside the new forming nucleus. Contrary to the two previous modes in this case, the concentration gradient assists the nucleation-in expression (1), coefficient $\gamma$ is negative. 
Above-mentioned approach was generalized taking into account the shape optimization [39-42], the stresses [43], ternary systems [44], heterogeneous nucleation at grain boundaries [41], at interphase interfaces [45]. Most simple models in the frame of this approach are presented in Section 4.

'Natural' thing is to expect that nature will use the mechanism with lowest nucleation barrier - the total mixing mode. Yet, nucleation is ruled not only by thermodynamics but by kinetics as well. Thermodynamics of nucleation with constraints indicates only some probable paths of evolution. Real path is chosen by kinetics, taking into account not only the free energy profit, but as well the different 'mobilities' along each path. Mobilities often appear to be inverse to the profit that is kind of compensation rule, analogous to relation between activation enthalpy and frequency factor in diffusion.

To calculate the nucleation kinetics, we used the Fokker-Planck approach, first applied to nucleation problems by Farkas [46] and recognized after classical work of Zeldovich [47]. Our contribution to this approach was just taking into account that the driving force depends on concentration gradient, which in its turn depends on time according to diffusion laws. It has been shown in [38] that the relative contribution of each mechanism depends on the ratio of atomic mobilities in the parent and nucleating phases. If atomic mobility in the new phase is much lower than in parent one, we can forget about total mixing mode. If opposite (high mobility inside new phase), then nucleation will proceed via total mixing, very fast ('fast is the first'). Main results are briefly reviewed in Section 5. One of the 'raisins' of total mixing (assisting) mode was that the easily formed nuclei, if not growing too fast, in comparison with decrease of concentration gradient, after some period can find themselves to be subcritical and be destroyed.

Further natural question was 'How does system proceed from nucleation of isolated particles to formation of continuous layer, which even initially appears much thicker (typically about $8 \mathrm{~nm}$ [48]) than the typical nucleus size?' Model of 'almost lateral' growth, driven by interface diffusion along the curved moving new phase boundaries, is briefly discussed in Section 6.

After discovering that concentration gradient can play a role of constraint on nucleation, it was natural for us to look for other constraints. Sharp concentration gradient means narrow layer, suitable for nucleation, e.g. limited volume. Therefore, the most natural thing was to treat the nucleation in small (nanosize) particles and the multiple simultaneous nucleations during formation of bulk nanocrystalline materials. Main result was as follows: depending on the volume of parent particle (or, in bulk, on the volume of 'responsibility region' around the nucleation site), the same 3 possibilities exist as in sharp concentration gradient-nucleation and growth (large size), metastable state (medium 
size), forbidden nucleation (small size). Results were published in [49, 50]. Afterwards we learned that similar results have been published by Rusanov 30 years before [51] and later developed in [52]. Yet, we hope that our application of these ideas to the 'traffic jam' effect in bulk glasses might be of some interest (Section 7): When many persons try to pass through narrow door simultaneously, the process stops. Similar effect can be responsible for long-living nanocrystalline states [53].

Most of above-mentioned problems are related to choosing a pathway for the evolution of a non-equilibrium system exhibiting phase transformations, whereby various regimes are possible for the same initial and boundary conditions. One more problem of this type, involving an invariant in the form of a product of the squared period of the growing structure and the growth rate, is how to predict both of these parameters for a discontinuous precipitation of a binary alloy with a given composition, which is supersaturated as a result of supercooling $[6,54]$. In contrast to a spinodal decomposition that takes place homogeneously in the whole volume and is controlled by changes in the elastic energy [55, 56], the transformation region represents a moving large-angle incoherent grain and phase boundary. A model of discontinuous precipitation in supercooled binary polycrystalline alloys at reduced temperatures, taking place because of the diffusion-induced grain-boundary migration, is constructed with allowance of grain boundary diffusion (Section 8). The approach based on the balance and maximum production of the entropy allows independent determination of the main parameters, including the interplate distance, the maximum velocity of the phase transformation front, and the concentration jump at this boundary [57].

One more unexpected field of 'nano-ideas' is a so-called anomalous mass-transfer under pulse loading of metals-transfer of atoms at distances of several microns, and sometimes much more, during shock lasting for less than hundred microseconds [58, 59]. Our recent molecular-dynamics simulation demonstrates the possibility of nanograin structure formation during the shock wave propagation in single crystals (Section 9). This result correlates with recent experimental results on shock loading of monocrystalline copper [60]. Existence (even virtual, during the shock) of nanograin structure can assist the masstransfer via mechanisms of grain-boundary or (that is more probable) 'mechanical diffusion' [61]. In Section 10, we discuss briefly some possible future developments.

\section{KINETIC MC SIMULATIONS}

\subsection{Direct MC Simulation of Reactive Diffusion}

Since in-situ observations of intermediate phase nucleation are lacking 
so far (to our knowledge), let us see what simulations can do.

Recently, we investigated the kinetics of ordering during interdiffusion and found the possibility of oscillatory ordering [62] (also see subsection 2.2). In that case, ordering was a second-order transition.

Simulation of first order transitions in square lattice has some difficulties. If one takes into account only the nearest neighbours interaction, then transition to B2-ordered phase is of second-order type: ordering without nucleation barrier. The formation of ordered intermediate phase is often related to change of electron subsystem, meaning the change of effective pair potentials of atomic interactions. To simulate first-order transitions, including nucleation of intermediate phase we modified our model making pair interactions extremely dependent on the local order. The simplest (and so far the most effective) model is as follows.

Model system is a 2-dimensional square lattice filled by $A$ and $B$ atoms with initial step-like distribution $\left(C_{B L}, C_{B R}\right)$ and periodic boundary conditions transforming diffusion couple into multilayer. Atoms $A$ are regarded as 'special' (and denoted as $A$ ") if they are surrounded by 4 neighbouring atoms $B$. The same is for $B^{*}$. These 'special' atoms have 'special' interactions with their neighbours. Our choice was as follows:

$$
\begin{gathered}
\frac{\Phi_{A A}}{k T}=\frac{\Phi_{B B}}{k T}=-3.0, \quad \frac{\Phi_{A B}}{k T}=-3.225, \\
\frac{\Phi_{A B^{*}}}{k T}=\frac{\Phi_{A^{*} B}}{k T}=\frac{\Phi_{A B}}{k T}+0.5, \quad \frac{\Phi_{A^{*} B^{*}}}{k T}=\frac{\Phi_{A B}}{k T}-2.0 .
\end{gathered}
$$

Migration of atoms was induced by vacancy migration. For each vacancy position, activation energies of exchange with each nearest neighbouring (NN) and next nearest neighbouring (NNN) atom were calculated as differences $\Delta E_{i}$ between the saddle-point energy and the energy in equilibrium site before jump. To simplify the model, we took all saddle-point energies to be equal to zero. Probability of each of 8 possible NN and NNN vacancy jumps was calculated as

$$
p=\frac{e^{-\frac{\Delta E_{i}}{k T}}}{\sum_{j=1}^{8} e^{-\frac{\Delta E_{j}}{k T}}}
$$

Subsequent MC simulation, using the residence-time algorithm [63], gave us full information about position of each atom after each MC step (MCS), including concentration profiles (averaged over $y$ for each $x$-plane), domains of full-order $A^{*} B^{*}$-particles of new phase, time dependence of ordered volume and of the system's energy [64].

Here, we show some results for two initial concentration steps- 

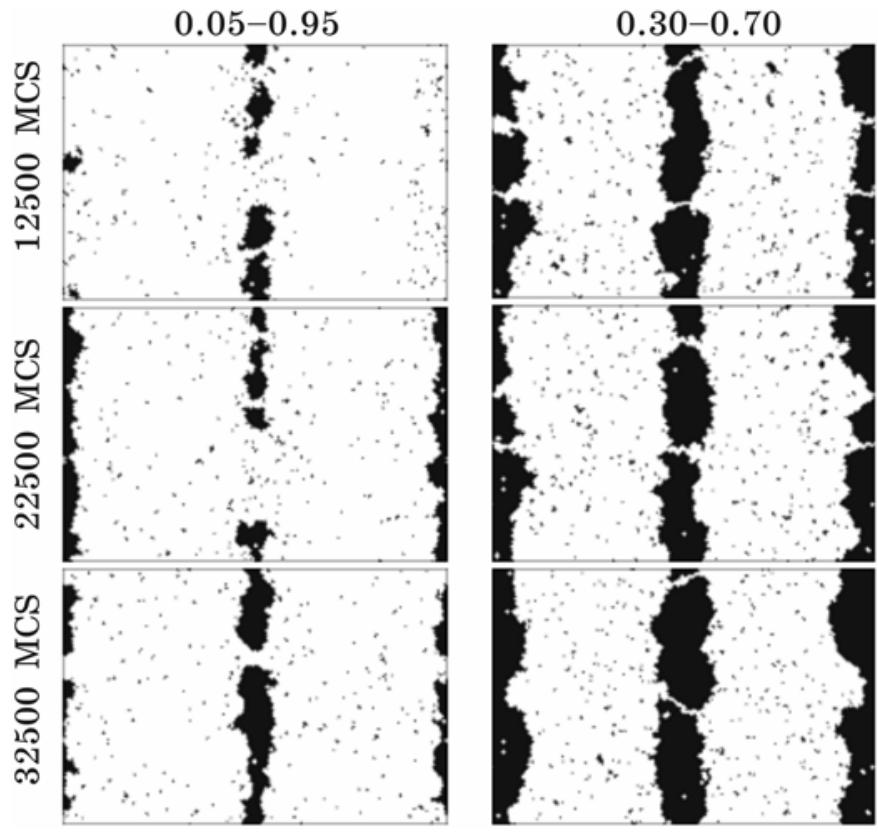

Fig. 2.1. Ordered intermediate phase formation in multilayer with initial concentration steps $C_{R}-C_{L}=0.90$ and 0.40 .

\section{(0.05-0.95) and (0.30-0.70) (Fig. 2.1).}

1. For big initial concentration step, a new phase growth has clearly oscillatory behaviour-antiphase domains of intermediate ordered phase appear and disappear, competing with each other and with parent phases. At the first stage, actually, all appearing nuclei eventually disappeared. Yet, with time some of them started surviving, which corresponds to our old nanve model of phase competition (the vampire phase being just the inhomogeneous solid solution with diffusivity much more than in the intermediate phase). In our model each domain is not permeable for vacancies, so that growth of new phase slows down after forming an initial layer, and further growth is possible due to 'channels' between antiphase domains.

2. Growth of new phase is faster for less initial concentration step. This result coincided with our theoretical prediction about suppression of new phase by the sharp concentration gradient [30, 31].

3. Phase growth clearly demonstrates the lateral regime, predicted by Coffey, Barmak et al. [14].

\subsection{Direct MC Simulation of Interdiffusion with Ordering}

It is interesting also to understand, how the interdiffusion proceeds at 
the very initial stage, if ordered phase does not need to overcome any nucleation barrier. In that case, we can observe the interplay of interdiffusion and ordering in concentration gradient. So, we used the same residence-time algorithm with constant pair interactions between the nearest neighbours in two-dimensional square lattice (triangular lattice was investigated as well). Energy of atom before jump was taken just as the sum of three energies (fourth neighbour being the vacancy). In the saddle-point of the jump to the nearest site, the atom is interacting with four neighbours. Our choice for pair energies was as follows:

for atoms in their sites, $\frac{\Phi_{A A}}{k T}=\frac{\Phi_{B B}}{k T}=-3, \frac{\Phi_{A B}}{k T}=-5$;

for jumping atom in the saddle point, $\Phi_{i j}^{\prime}=B \cdot \Phi_{i j}, B=0.5$.

Initial distribution of atoms corresponded to diffusion couple with different concentrations on the 'left' and 'right'-concentration step. Initial profiles with concentrations steps $0.00-1.00,0.20-0.80,0.40-$ 0.60 and $0.50-0.50$ (homogeneous case) were simulated.

Results were a little bit unexpected (for details see [62]):

1. Formation of ordered domains in the couples with nonzero concentration steps clearly demonstrated nonmonotonic behaviourblinking. Domains appeared, lived for some time and then disappeared. Blinking was more pronounced for samples with larger initial concentration steps.

2. The total area of ordered phase, of course, eventually grew with time, but not monotonically: the time dependence of the ordered region's area with order parameter above 0.9 showed nonmonotonic stochastic behaviour. To distinguish the usual statistical noise of MC procedure from real order oscillations, we used the coarsening of the time scale. Choice of coarsening scale was made in such a way, that it provided monotonic ordering of initially homogeneous samples (couples 0.5-0.5). The results are shown in Fig. 2.2.

To explain these rather unexpected results, we proposed the following picture. Diffusivity strongly depends on the order parameter. Therefore, the newly formed ordered regions, though energetically favourable, have substantially less diffusivity than the surrounding parent disordered solid solution. Hence, they start to 'loose their game' in diffusive competition with neighbouring phase. Therefore, we can observe the competition of two factors-thermodynamic profit and kinetic disability leading to oscillations. We tried to check, if such explanation is reasonable, by means of the following simplified phenomenological model of simultaneous interdiffusion and ordering with nonlinear dependence of diffusivity on the order parameter:

$$
\frac{\partial c}{\partial t}=\frac{\partial}{\partial x}\left(\tilde{D}(\eta) \frac{\partial c}{\partial t}\right)
$$



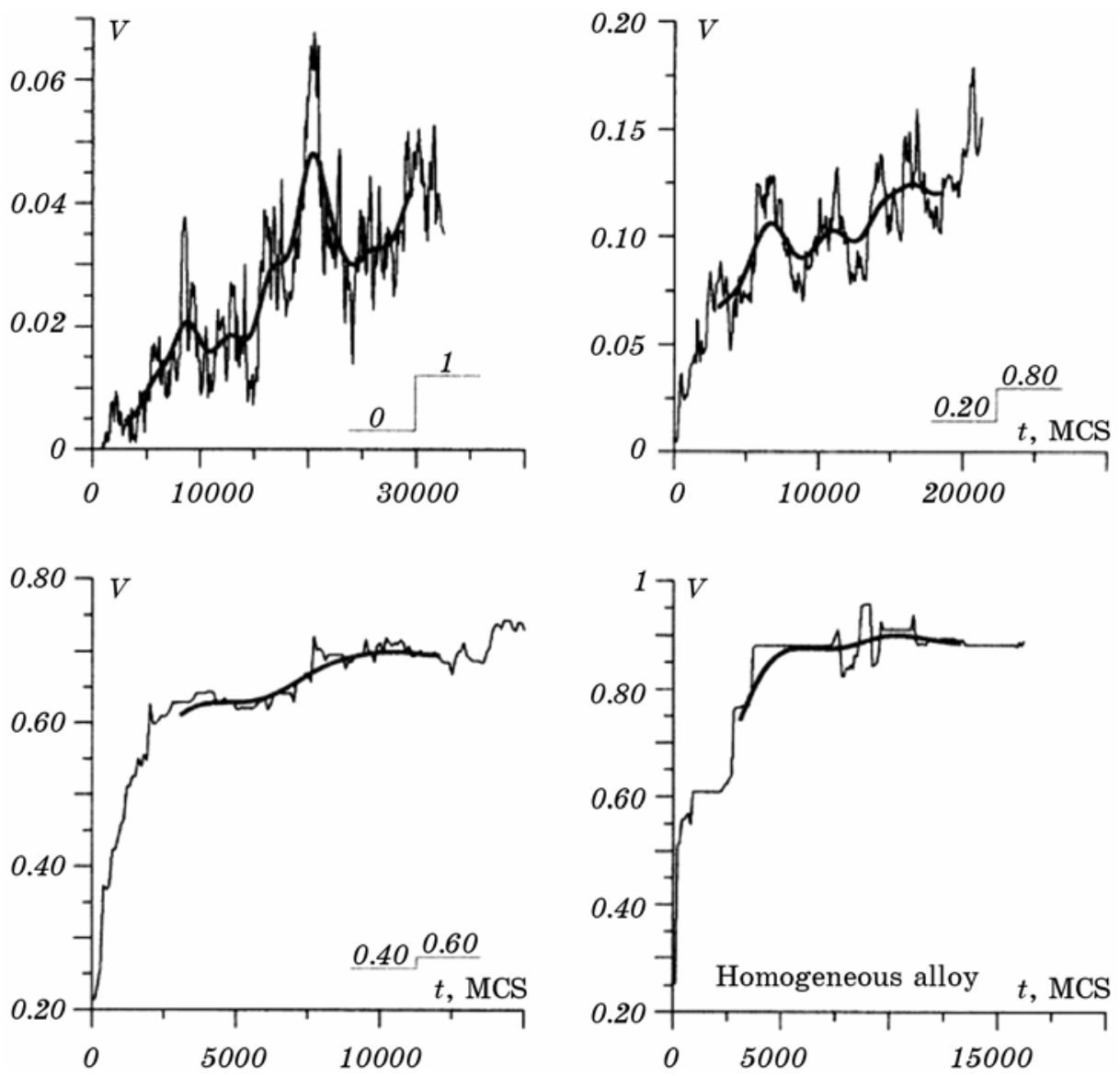

Fig. 2.2. Time dependence of the ordered 'volume' fraction (with absolute value of order parameter larger than 0.9) in the planar layer of the diffusion zone containing 40 atom rows. Time is measured in Monte Carlo steps (MCS).

$$
\frac{\partial \eta}{\partial t}=-\gamma\left(\eta-\eta_{e}(c)\right)
$$

where $\eta_{e}(c)$ is an equilibrium value of order parameter $\eta$ corresponding to local value of concentration.

Dependence $\tilde{D}(\eta)$ was proposed as quadratic under exponent, in the frame of mean-field considerations for the energy of jumping atoms in the sites:

$$
\tilde{D}(\eta)=D_{0} \exp \left(-\alpha \eta^{2}\right)
$$

with $\alpha$ being proportional to the mixing energy. 


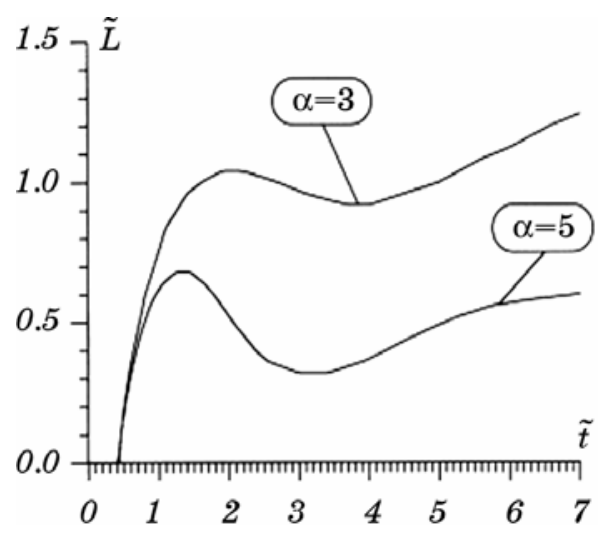

Fig. 2.3. Time dependences of ordered region's size $(\eta>0.3)$ for $D_{0}=1$; $\gamma=1 ; \alpha=3$, 5. Initial concentration step is $0.00-1.00$.

Numeric solution of the problem (2.2)-(2.4) demonstrated a possibility of nonmonotonic ordering for the certain region of parameters $D_{0}, \gamma, \alpha$. Characteristic time dependences of ordered (with $\eta>0.3$ ) region's size for dimensionless values $D_{0}=1, \gamma=1, \alpha=3,5$ are shown in Fig. 2.3. Increase of parameter $\alpha$ leads to more pronounced oscillation of order. These oscillations depend also on the initial concentration step: the larger is this step, the more pronounced are the oscillations (similar to MC simulations); see Fig. 2.4.

Thus, the main results of phenomenological model coincide with MC results, except the number of oscillations. We believe the reason is just one-dimensional character of the phenomenological formulation.

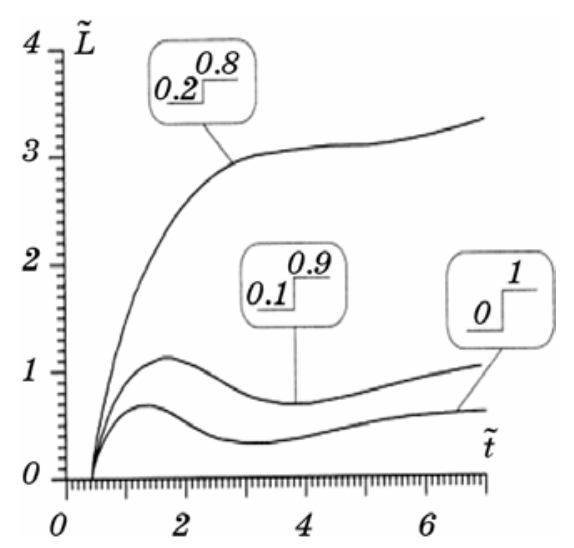

Fig. 2.4. Time dependences of the ordered region's size for different initial concentration steps; $D_{0}=1, \gamma=1, \alpha=5$. 


\section{PHASE COMPETITION}

As we have just seen in MC simulations, a phase growth in diffusion zone starts with the nucleation of intermediate phases as a result of heterophase fluctuations. A nucleus is thermodynamically stable if its size exceeds the critical value (if the gain in bulk free energy due to attachment of monomers begins to exceed the loss in surface energy). The fundamental difference from the phase formation by alloy cooling is as follows: the phase nuclei are formed in the field of chemicalpotential gradients, and therefore, at once begin to interact diffusionally with parent phases and/or with the nuclei of other intermediate phases. This diffusional interaction is mathematically described by the system of balance equations for the fluxes on the moving interphase boundaries. As a result, the growth velocity of some critical nuclei appears to be negative, they start decreasing, transforming into embryos with subcritical size. Such embryos are thermodynamically unstable and must dissociate. It means that certain intermediate phases from the phase diagram of the system can be absent in the diffusion zone for some time (which can be a rather long time). To be more precise such suppressed phases are virtually present, in the form of forming, decreasing and dissociating nuclei. The corresponding criteria of phase growth/suppression were obtained.

\subsection{Simple Phenomenological Model of Diffusive Phase Competi- tion of the Two Phases}

Consider binary diffusion couple $A-B$ with two intermediate phases 1 , 2 , and negligible solubilities of $A$ in $B$ and of $B$ in $A$. If, by some reasons, only phase layer 1 grows in diffusion zone, then its growth law is:

$$
\Delta x_{1}=\sqrt{\frac{2 D_{1} \Delta C_{1}}{C_{1}\left(1-C_{1}\right)} t}
$$

( $\Delta C_{1}$-concentration range of the 1 -st phase, effective diffusivity $\left.D_{1} \equiv \frac{1}{\Delta C_{1}} \int_{\Delta C_{1}} \tilde{D}(C) d C \quad[61,62]\right), \quad \tilde{D}(C)$-interdiffusion coefficient).

Since the reaction $1+B \rightarrow 2$ is thermodynamically favourable, the phase 2 should form the critical nuclei with longitudinal size $l_{2}$ at the moving interface $1-B$ due to heterophase fluctuations. As will be shown below, sharp concentration gradients lead to the plate-like shape of the nuclei. Therefore, one can consider left and right boundaries of the nucleus as nearly flat. Steps of the diffusion flux profiles at these boundaries generate their movement. According to conservation 
of matter at the moving boundaries,

$$
\left(C_{2}-C_{1}\right) \frac{d x_{2 L}}{d t}=\frac{D_{1} \Delta C_{1}}{\Delta x_{1}}-\frac{D_{2} \Delta C_{2}}{l_{2}},\left(1-C_{2}\right) \frac{d x_{2 R}}{d t}=\frac{D_{2} \Delta C_{2}}{l_{2}}-0
$$

(we neglect solubility of $A$ in $B$ and assume the regions of homogeneity to be narrow).

Hence, growth/shrinkage rate of critical nucleus' width is equal to

$$
\left.\frac{d \Delta x_{2}}{d t}\right|_{l_{2}}=\frac{d x_{2 R}}{d t}-\frac{d x_{2 L}}{d t}=\frac{1}{C_{2}-C_{1}}\left(-\frac{D_{1} \Delta C_{1}}{\Delta x_{1}}+\frac{1-C_{1}}{1-C_{2}} \frac{D_{2} \Delta C_{2}}{l_{2}}\right) .
$$

One can easily see that this expression is positive for big $\Delta x_{1}$, but can be negative for small $\Delta x_{1}$.

If $\Delta x_{1}<\Delta x_{1}^{*}=\frac{1-C_{2}}{1-C_{1}} \frac{D_{1} \Delta C_{1}}{D_{2} \Delta C_{2}} l_{2}$, then $\left.\frac{d \Delta x_{2}}{d t}\right|_{l_{2}}<0$, so that every critical nucleus decreases (being 'eaten' by rapidly growing neighbouring phase 1) and therefore becomes subcritical embryo (unstable), and should be dissolved.

Such unsuccessful attempts of phase- 2 nucleation will be repeated during some 'incubation period' $\tau$, till the suppressing phase 1 reaches thickness $\Delta x_{1}^{*}$ growing according to parabolic law (3.1):

$$
\tau=\frac{C_{1}\left(1-C_{1}\right)}{2 D_{1} \Delta C_{1}}\left(\Delta x_{1}^{*}\right)^{2}=\frac{C_{1}\left(1-C_{2}\right)^{2}}{2\left(1-C_{1}\right)} \frac{D_{1} \Delta C_{1}}{\left(D_{2} \Delta C_{2}\right)^{2}} l_{2}^{2} .
$$

Of course, Eq. (3.4) can be used only if the diffusion couple is sufficiently large. If the specie $B$ is consumed before phase-1 layer could reach the critical thickness $\Delta x_{1}^{*}$, the phase 2 will never appear at all.

So far, we just assumed that it is phase 1, which grows first. To determine which of phases will actually grow first, one should consider diffusive interaction between two initial layers of critical nuclei of both phases. One can easily check that

$$
\begin{gathered}
\left.\frac{d \Delta x_{1}}{d t}\right|_{l_{1}}=\frac{1}{C_{2}-C_{1}}\left(\frac{C_{2}}{C_{1}} \frac{D_{1} \Delta C_{1}}{l_{1}}-\frac{D_{2} \Delta C_{2}}{l_{2}}\right), \\
\left.\frac{d \Delta x_{2}}{d t}\right|_{l_{2}}=\frac{1}{C_{2}-C_{1}}\left(-\frac{D_{1} \Delta C_{1}}{l_{1}}+\frac{1-C_{1}}{1-C_{2}} \frac{D_{2} \Delta C_{2}}{l_{2}}\right) .
\end{gathered}
$$

System has three possibilities:

1) $\frac{D_{1} \Delta C_{1}}{D_{2} \Delta C_{2}} \frac{l_{2}}{l_{1}}<\left.\frac{C_{1}}{C_{2}} \Rightarrow \frac{d \Delta x_{1}}{d t}\right|_{l_{1}}<0,\left.\frac{d \Delta x_{2}}{d t}\right|_{l_{2}}>0$, i.e. phase 2 ('vampire') 
starts growing, 'eating' the nuclei of the phase 1;

2) $\frac{C_{1}}{C_{2}}<\frac{D_{1} \Delta C_{1}}{D_{2} \Delta C_{2}} \frac{l_{2}}{l_{1}}<\left.\frac{1-C_{1}}{1-C_{2}} \Rightarrow \frac{d \Delta x_{1}}{d t}\right|_{l_{1}}>0,\left.\frac{d \Delta x_{2}}{d t}\right|_{l_{2}}>0$, i.e. both phases grow from the moment of nucleation;

3) $\frac{D_{1} \Delta C_{1}}{D_{2} \Delta C_{2}} \frac{l_{2}}{l_{1}}>\frac{1-C_{1}}{1-C_{2}}$, i.e. phase 1 ('vampire') starts growing, 'eating' the nuclei of phase 2 .

Phase suppression cannot continue infinitely. As the suppressing ('vampire') phases grow, the concentration gradients and corresponding fluxes along the phase layers decrease, their growth velocity slows down and their 'competition ability' also decreases, For every suppressed phase the moment exists (see Eq. (3.4)), when the growth velocity of its critical nuclei becomes positive, and they start to grow. The time of diffusion suppression of the phase nuclei is called the incubation period. Evidently, it is only the 'diffusion' part of the full incubation period. The process mentioned above was called diffusion phase competition. Such a simple approach is easily generalized on the arbitrary number of intermediate phases [31].

\section{THERMODYNAMICS OF NUCLEATION IN CONCENTRATION GRADIENT}

So far, we used classic nucleation theory. Now, we reconsider thermodynamic of nucleation in the contact zone.

\subsection{Polymorphous Nucleation Mode}

Let the nucleus of intermediate phase appear in the frozen concentration profile formed by interdiffusion in the metastable continuous phase (solid solution or amorphous phase) (Fig. 4.1). If concentration gradient is sharp enough (narrow diffusion zone), the driving force of nucleation per atom $\Delta g_{a}$ (function of composition) becomes the function of space coordinates as well.

Hence, the change of Gibbs free energy caused by formation of nucleus in such sharply inhomogeneous conditions is a sum of different contributions from each thin slice $S(x) d x$ and is given by the following expression:

$$
\Delta G=n \int\left(g^{\text {new }}(C(x))-g^{\text {old }}(C(x))\right) S(x) d x+\sigma S .
$$

Here, we neglect the volume changes (atomic density $n_{1} \approx n_{2}=n$ ) and the corresponding stresses. $S$ is the area of newly born interface surface, $\sigma$-its surface energy per unit area, $S(x)$-area of nucleus cross- 


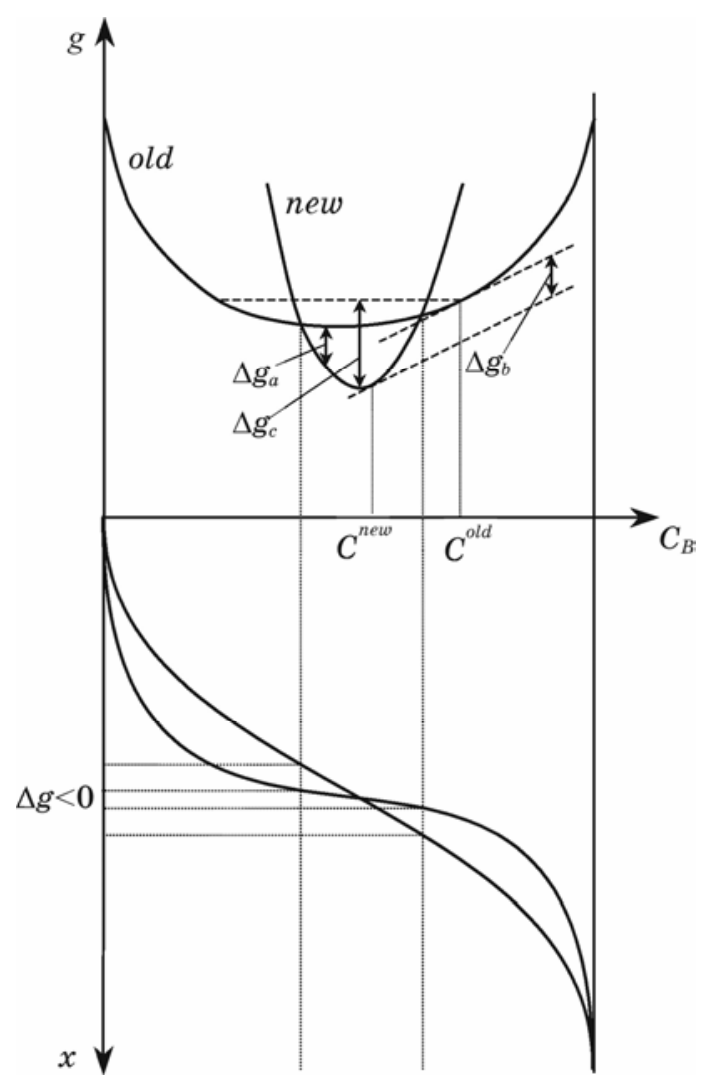

Fig. 4.1. 'Gibbs free energy versus composition' and corresponding 'composition versus diffusion co-ordinate' dependences. Driving forces per atom of nucleus are indicated for $(a)$ polymorphous, $(b)$ transversal and $(c)$ total mixing modes.

section with plane $x$, perpendicular to concentration gradient, $g-$ Gibbs free energy per one atom. To make mathematics simpler, the following approximations are suggested:

$$
\begin{gathered}
g^{\text {old }}(C)=g_{0}^{\text {old }}+\frac{\alpha^{\text {old }}}{2}\left(C-C_{0}^{\text {old }}\right)^{2}, g^{\text {new }}(C)=g_{0}^{\text {new }}+\frac{\alpha^{\text {new }}}{2}\left(C-C_{0}^{\text {new }}\right)^{2} \\
C(x) \cong C(0)+x \cdot \nabla C .
\end{gathered}
$$

Taking into account Eqs. (4.2), (4.3), one obtains:

$$
\Delta G=n \int\left(A_{0}+A_{1} \nabla C \cdot x+A_{2}(\nabla C)^{2} x^{2}\right) S(x) d x+\sigma S,
$$

where 


$$
\left\{\begin{array}{l}
A_{0}=-\left(g_{0}^{\text {old }}-g_{0}^{\text {new }}+\frac{1}{2}\left[\alpha^{\text {old }}\left(C(0)-C_{0}^{\text {old }}\right)^{2}-\alpha^{\text {new }}\left(C(0)-C_{0}^{\text {new }}\right)^{2}\right]\right), \\
A_{1}=\alpha^{\text {new }}\left(C(0)-C_{0}^{\text {new }}\right)-\alpha^{\text {old }}\left(C(0)-C_{0}^{\text {old }}\right), A_{2}=\left(\alpha^{\text {new }}-\alpha^{\text {old }}\right) / 2 .
\end{array}\right.
$$

\subsubsection{Spherical Nuclei}

Let nucleus be the sphere with the centre in some point $x_{C}$, so that

$$
S(x)=\pi\left(R^{2}-\left(x-x_{C}\right)^{2}\right) .
$$

Then simple algebra transforms Eq. (4.1) into

$$
\Delta G=\sigma \cdot 4 \pi R^{2}+n \pi\left(B_{0} \frac{4}{3} R^{3}+B_{2} \frac{4}{15} R^{5}\right),
$$

where

$$
B_{0}=A_{0}+A_{1} x_{C} \nabla C+A_{2} x_{C}^{2}(\nabla C)^{2}, \quad B_{2}=A_{2}(\nabla C)^{2} .
$$

First of all one should find an optimal place for nucleation from the conditions

$$
\begin{gathered}
\frac{\partial \Delta G}{\partial x_{C}}=0, \\
\frac{\partial^{2} \Delta G}{\partial x_{C}^{2}}>0: \\
x_{C}=-\frac{A_{1}}{2 A_{2} \nabla C}=\frac{\alpha^{\text {old }}\left(C(0)-C_{0}^{\text {old }}\right)-\alpha^{\text {new }}\left(C(0)-C_{0}^{\text {new }}\right)}{\left(\alpha^{\text {new }}-\alpha^{\text {old }}\right) \nabla C}
\end{gathered}
$$

(it corresponds to the minimum, if $\alpha^{\text {new }}>\alpha^{\text {old }}$ ).

Thus, with changing concentration gradient time the optimal place of nucleation shifts but the corresponding concentration in the centre

$$
C(0)+x_{C} \nabla C=C(0)-\frac{A_{1}}{2 A_{2}}
$$

remains the same.

Further, we restrict ourselves only to nuclei forming in the optimal place. In this case, the size dependence of $\Delta G$ has a simple form:

$$
\Delta G(R)=\alpha R^{2}-\beta R^{3}+\gamma(\nabla C)^{2} R^{5},
$$


where

$$
\left\{\begin{array}{l}
\alpha=4 \pi \sigma, \quad \gamma=\frac{4 \pi n}{15} \frac{\alpha^{\text {new }}-\alpha^{\text {old }}}{2}, \\
\beta=\frac{4 \pi n}{3}\left(\frac{A_{1}^{2}}{4 A_{2}}-A_{0}\right)=\frac{4 \pi n}{3}\left(g_{0}^{\text {old }}-g_{0}^{\text {new }}+\frac{\alpha^{\text {new }}-\alpha^{\text {old }}}{2\left(\alpha^{\text {new }}-\alpha^{\text {old }}\right)}\left(C_{0}^{\text {old }}-C_{0}^{\text {new }}\right)^{2}\right) .
\end{array}\right.
$$

Coefficient $\beta>0$, if the curve $g^{\text {new }}(C)$ intersects the curve $g^{\text {old }}(C)$.

As follows from Eq. (4.8), the dependence $\Delta G(R)$ can be monotonic or nonmonotonic, depending on the magnitude of concentration gradient (Fig. 4.2).

Case $(a)$ corresponds to full suppression of nucleation at sharp concentration gradients. Case $(b)$ means a possibility of metastable nucleus formation. Case $(c)$ means the possibility of forming the stable particle of new phase, size of which will increase with decreasing (with time due to interdiffusion) concentration gradient.

Simple algebra gives the following expressions for the values

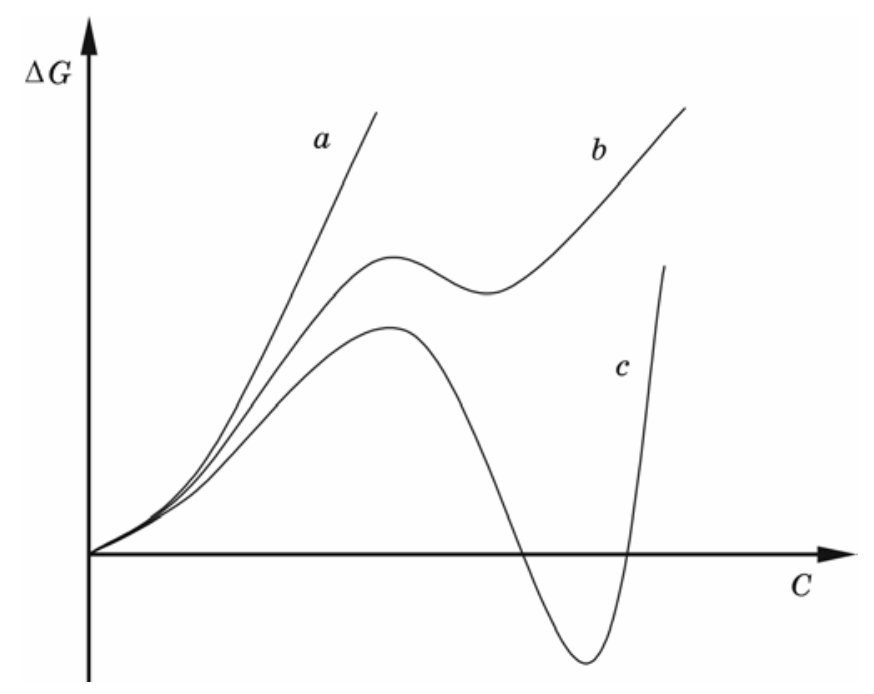

Fig. 4.2. Size dependence for Gibbs free energy change by nucleation of spherical particles in the concentration gradient.

(a) $\nabla C>(\nabla C)_{1}^{\text {crit }}=\frac{\beta}{\alpha} \sqrt{\frac{\beta}{5 \gamma}}-$ nucleation forbidden;

(b) $(\nabla C)_{1}^{\text {crit }}>\nabla C>(\nabla C)_{2}^{\text {crit }}=\frac{\beta}{\alpha} \sqrt{\frac{4 \beta}{27 \gamma}}-$ possibility of metastable nucleus;

(c) $\nabla C<(\nabla C)_{2}^{\text {crit }}$-nucleation possible. 
$(\nabla C)_{1,2}^{\text {crit }}$, corresponding to crossovers $b \leftrightarrow a$ and $c \leftrightarrow b$ :

$$
(\nabla C)_{1}^{\text {crit }}=\frac{\beta}{\alpha} \sqrt{\frac{\beta}{5 \gamma}}, \quad(\nabla C)_{2}^{\text {crit }}=\frac{\beta}{\alpha} \sqrt{\frac{4 \beta}{27 \gamma}} .
$$

One can see the values of $(\nabla C)_{1}^{\text {crit }}$ and $(\nabla C)_{2}^{\text {crit }}$ are rather close, so that the regime of metastable nucleation is difficult to detect. Moreover, we shall see below that the shape optimisation excludes this regime (if one does not consider stresses). Numerical estimates for the critical gradient for systems like Ni-Zr typically give $(\nabla C)_{1}^{\text {crit }} \propto 10^{8} \mathrm{~m}^{-1}$.

\subsubsection{Shape Optimisation}

Evidently, since concentration gradient suppresses the nuclei growth in longitudinal direction, nature will find possibilities to increase nucleus volume (and decrease Gibbs free energy) by transversal growth. It means that nuclei forming in the diffusion zone should be nonspherical. For each fixed nucleus volume, one should take into account the shape optimisation. First attempt in this direction was made in 1991 [39]. Nuclei (embryo) were supposed to be spheroids with symmetry (rotation) axis along the $\nabla C$-direction with parameters $R_{\|}(\| x)$ and $R_{\perp}(\perp x)$. In this case, $\nabla G$ is a function of two arguments - volume $V$ and shape parameter $\eta=R_{\perp} / R_{\|}$at fixed concentration gradient $\nabla C=1 / L$ :

$$
\begin{aligned}
\Delta G\left(R_{\|}, R_{\perp}\right)=2 \pi n\{ & -\frac{2}{3}\left(\Delta g_{0} R_{\|} R_{\perp}^{2}+\frac{g^{\prime \prime}}{15 L^{2}} R_{\|}^{3} R_{\perp}^{2}+\frac{2 \sigma}{n}\left[\frac{R_{\perp}^{2}}{2}+\frac{R_{\perp} R_{\|}^{2}}{2 \sqrt{R_{\perp}^{2}-R_{\|}^{2}}}\right) \times\right. \\
& \times\left\{\begin{array}{l}
\ln \left(\sqrt{\left.\left.\left.\left(\frac{R_{\perp}}{R_{\|}}\right)^{2}-1+\frac{R_{\perp}}{R_{\|}}\right)\right]\right\}, \frac{R_{\perp}}{R_{\|}}>1,}\right. \\
\left.\left.\arcsin \sqrt{1-\left(\frac{R_{\perp}}{R_{\|}}\right)^{2}}\right]\right\}, \frac{R_{\perp}}{R_{\|}}<1,
\end{array}\right.
\end{aligned}
$$

where $R_{\|}=\left(\frac{3 V}{4 \pi}\right)^{\frac{1}{3}} \cdot \eta^{-\frac{2}{3}}, R_{\perp}=\left(\frac{3 V}{4 \eta}\right)^{\frac{1}{3}} \cdot \eta^{\frac{1}{3}}$.

At every fixed volume $V$, an optimal shape $\eta(V)$ is found by minimizing the function $\Delta G(V \mid \eta)$. The function $\eta_{\text {opt }}(V)$ increases to infinity 


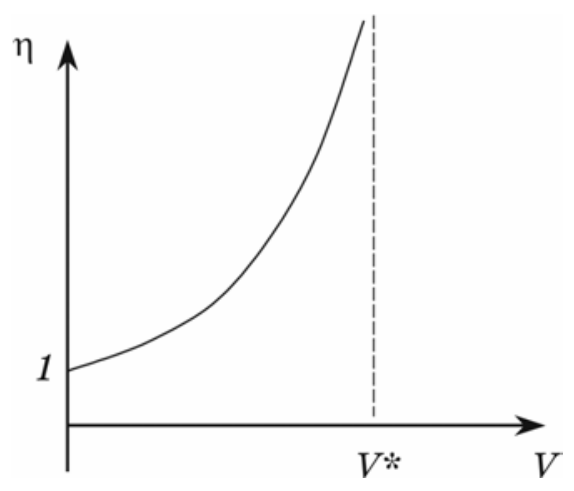

Fig. 4.3. Dependence of optimal shape, $\eta=R_{\perp} / R_{\|}$, on nucleus volume.

at some value $V^{*}$ (Fig. 4.3), which is determined by the concentration gradient (the larger is $\nabla C$, the less is $\left.V^{*}\right)$. Dependence $\Delta G\left(V, \eta_{\text {opt }}(V)\right)$ looks different for large and for small concentration gradients (Fig. 4.4).

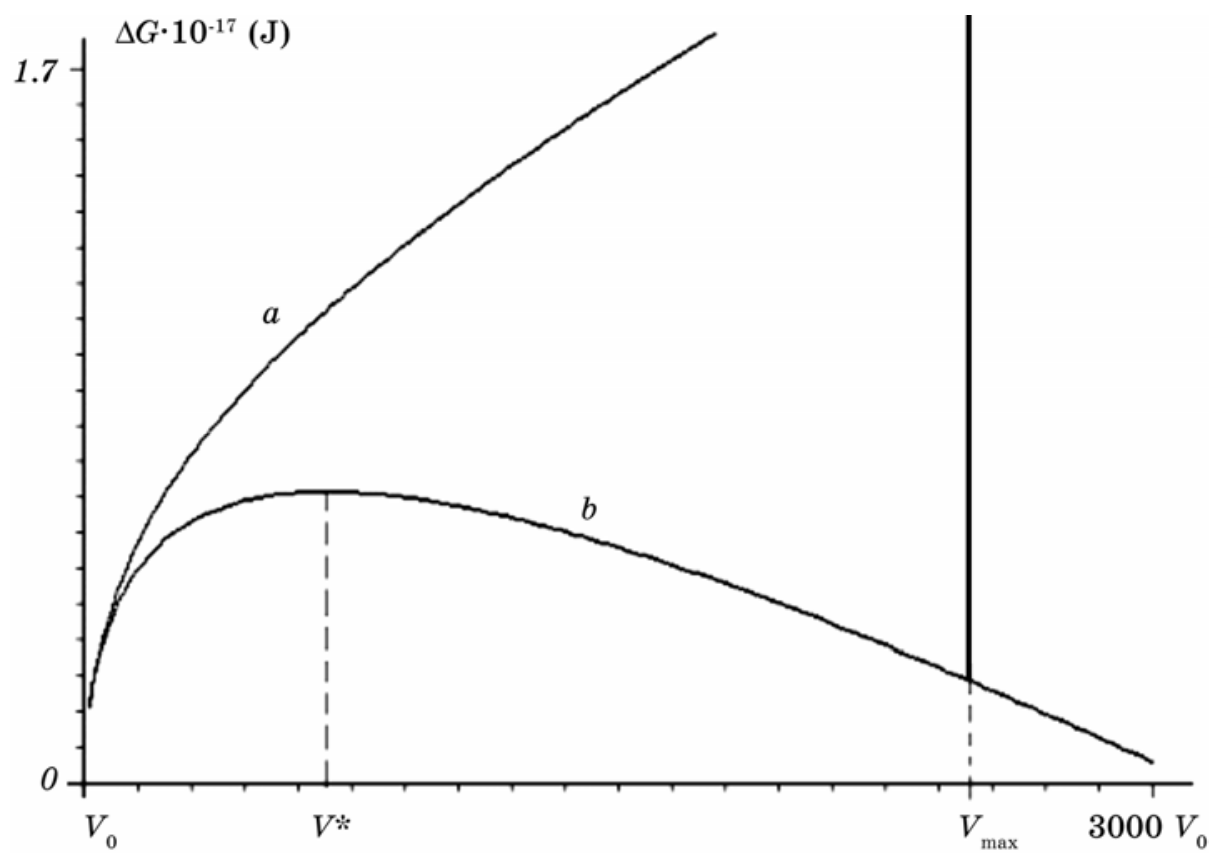

Fig. 4.4. Dependence of Gibbs free energy on the volume of nucleus with optimized shape: $(a) \nabla C>(\nabla C)^{\text {crit }} ;(b) \nabla C<(\nabla C)^{\text {crit }} . V_{0}=3 \cdot 10^{-29} \mathrm{~m}^{3}, \Delta g_{o}=10^{-20} \mathrm{~J} ; \sigma=$ $=0.5 \mathrm{~J} / \mathrm{m}^{2} ; n=10^{29} \mathrm{~m}^{-3} ; g^{\prime \prime}=10^{-16} \mathrm{~J}$, critical width of diffusion zone appeared to be $L^{\text {crit }}=1 / \nabla C^{\text {crit }}=6.2 \cdot 10^{-8} \mathrm{~m}, V_{\max }$ corresponds to some volume at which the growing nucleus meets another laterally growing nucleus and stops the own lateral growth. 
Thus, the main result of previous subsection is repeated-the existence of concentration gradient, over which nucleation of intermediate phase is forbidden. Yet, the possibility of metastable nuclei disappeared due to shape optimisation.

New results are: (1) the formation of plate-like nuclei (of course, this result is not valid, if critical $\Delta G$ is much higher than $60 k T$ ) and (2) decrease of nucleation barrier and corresponding increase of the value of critical concentration gradient due to the shape optimisation.

Of course, suggestion of spheroidal shape is not rigorous. To verify the validity of the above-mentioned results, we will present the direct Monte Carlo simulation of the nucleus formation.

\subsubsection{Simulation of Nucleus Shape in Concentration Gradient}

We investigated the possible nucleation of the stable intermediate phase in concentration gradient by means of Monte Carlo technique. Let the concentration dependences of Gibbs potential for both phases to be approximately parabolic with minima at $C_{0}^{\text {new }}=C_{0}^{\text {old }}=1 / 2$. Concentration profile in the parent phase in the vicinity of the forming nucleus is approximated by linear dependence. In polymorphous mode, nucleation proceeds fast in the frozen concentration gradient, and concentration changes start due to diffusion after nucleation. We divide the homogeneous alloy into 'elementary' cells, each of them can transform from old to new phase and vice versa, depending on thermodynamic profit, which is determined by bulk driving force and the number of neighbouring cells in different phase states.

Simulation procedure. Each cell can exist in two-phase states-old and new. The change of the phase state leads to change of bulk and surface energy. For example, if the cell transforms from old to new state then the change of Gibbs potential for the system is equal to

$$
\Delta G=\left(g^{\text {new }}(C)-g^{\text {old }}(C)\right) \cdot\left(a^{3} n\right)+\sigma \Delta N a^{2} .
$$

Here $C$ is the concentration in the cell depending on its position ( $x-$ coordinate, if the concentration gradient is parallel to $x$ ), $n$-atomic density, $a$-the cell size, $\sigma$-surface tension between old and new phases, $\Delta N$ is a change of number of neighbouring cells with different states (even number from -6 to +6 ). If $\Delta G$ is negative, the transformation is accepted, otherwise acceptance probability is found as $\exp (-\Delta G / k T)$ (Metropolis algorithm). To make the Monte Carlo procedure time saving, we try the state changes only for cells in the border layer of the forming nucleus.

The following algorithm has been realized.

0) At first, all cells belong to old phase. Randomly we choose one cell as a nucleation site and try its transformation according to Metropolis 
procedure $(\Delta N=6)$.

1) One of the border cells is chosen randomly from the border set. (Cell belongs to border set if it belongs to nucleus and has at least one neighbour of old phase).

2) Cluster consisting of the chosen border cell and its six neighbours is further considered. One of the seven cells of this cluster is chosen randomly. This choice is accepted if the chosen cell is central (transformation new $\rightarrow$ old) or if it is a neighbour belonging to old phase (transformation old $\rightarrow$ new). Otherwise, attempt is repeated.

3) Change of the Gibbs potential for possible transformation is calculated according to Eq. (4.12) and decision on acceptance/non-acceptance is made according to the Metropolis procedure.

Step (2) of the abovementioned algorithm artificially increases the probability of nucleus growth. Otherwise, the subcritical embryo would be most probably destroyed, and the formation of overcritical nucleus would take very long computation time. The results of simulation are presented for sharp and for small concentration gradients at Fig. 4.5.
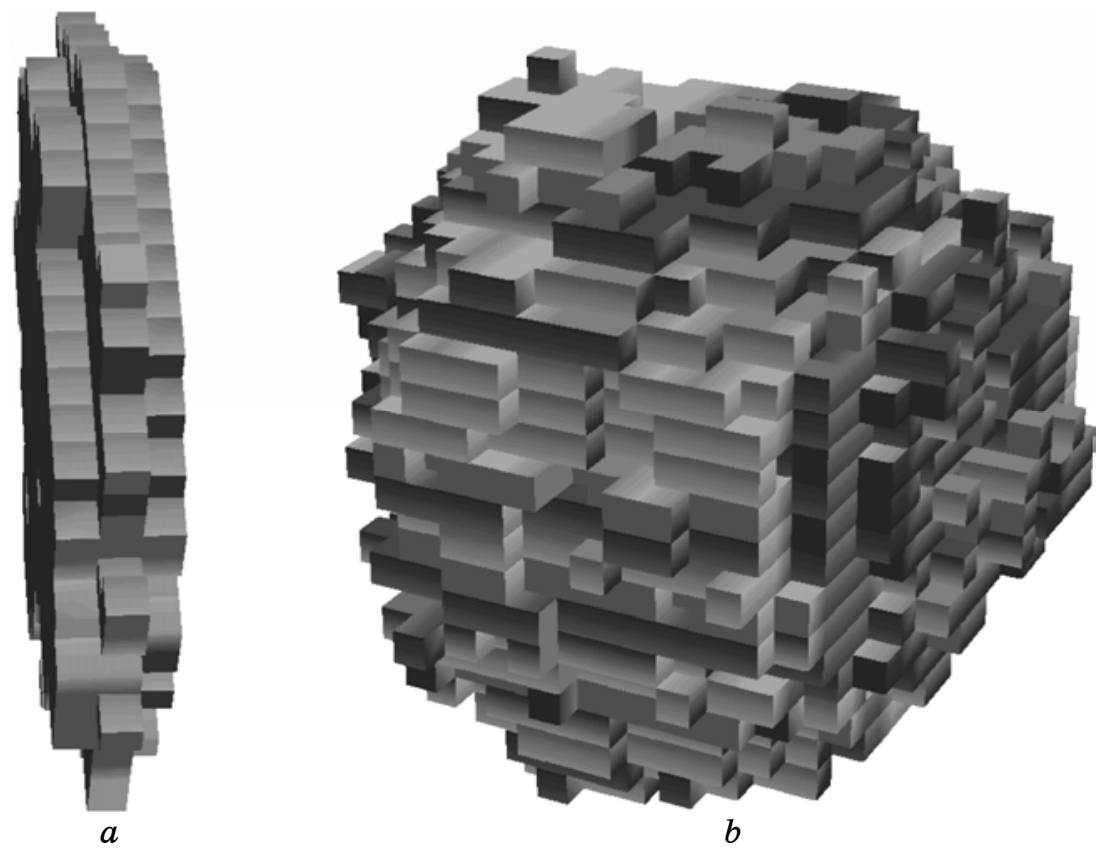

Fig. 4.5. Examples of nucleus shape simulation for $\operatorname{sharp}\left((a)-\nabla C=10^{9}\right.$ $\left.\mathrm{m}^{-1}\right)$ and small $\left((b)-\nabla C=10^{7} \mathrm{~m}^{-1}\right)$ concentration gradients. Parameters of simulation: $\sigma=0.15 \mathrm{~J} / \mathrm{m}^{2}, a=1.5 \cdot 10^{-10} \mathrm{~m}, n=10^{29} \mathrm{~m}^{-3}, \frac{\partial^{2} \Delta g}{\partial C^{2}}=7.77 \cdot 10^{-19} \mathrm{~J}$, $g_{0}{ }^{\text {old }}-g_{0}{ }^{\text {new }}=7.48 \cdot 10^{-21} \mathrm{~J}$. 


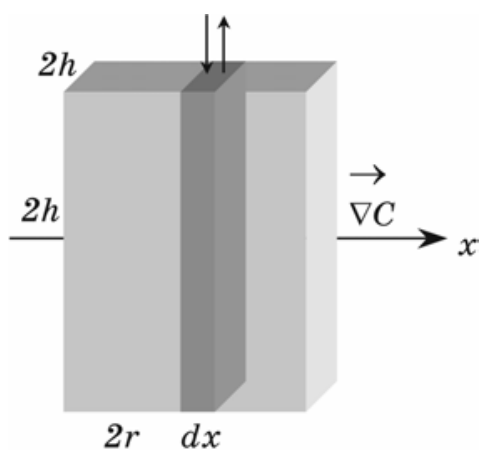

Fig. 4.6. Scheme of transversal nucleation modes, vertical arrows show the direction of redistribution fluxes of species in the slice $d x$.

\subsection{Transversal Nucleation Mode}

P. Desré and Yavari [32] first introduced this mode for cubic nuclei without shape optimisation. In 1998, shape optimisation was done by F. Hodaj, A. Gusak, and P. Desré [37] for the simplest case of parallelepipeds. Let embryo (nucleus) in the form of parallelepiped $2 h \times 2 h \times 2 r$ is born in the concentration gradient $\nabla C$ of metastable parent phase ( $2 r$ along $\nabla C$ ). Every thin slice $2 h \times 2 h \times d x$ with concentration $C^{\text {new }}(x)$ forms at the cost of slice $\infty \times \infty \times d x$ with concentration $C^{\text {old }}(x)$ according to the rule of parallel tangents (Fig. 4.7).

Driving force per atom of nucleus (not of total system) is equal to

$$
\Delta g_{b}=g^{\text {old }}\left(C^{\text {old }}\right)+\frac{\partial g}{\partial C}\left(C^{\text {new }}-C^{\text {old }}\right)-g^{\text {new }}\left(C^{\text {new }}\right)
$$

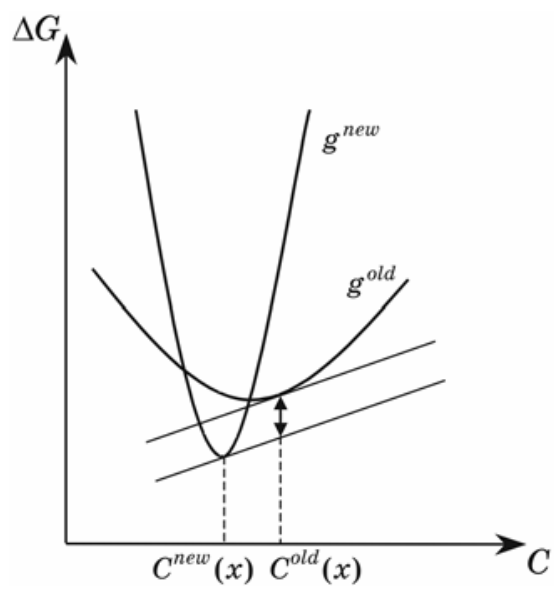

Fig. 4.7. Rule of parallel tangents. 
and is determined by vertical interval between two parallel tangents as shown on Fig. 4.7.

Using the rule of parallel tangents for each thin slice $d x$ of parallelepiped, one obtains:

$$
\Delta G=-n \int_{x_{C}-r}^{x_{C}+r} \Delta g\left(C^{\text {old }}(x) \rightarrow C^{\text {new }}(x)\right) \cdot 4 h^{2} d x+2 \sigma_{1} \cdot 4 h^{2}+4 \sigma_{2} \cdot 4 h r,
$$

where $C^{\text {old }}(x)=C(0)+x \cdot \nabla C, \quad C^{\text {new }}(x)=C_{0}^{\text {new }}+\frac{\alpha^{\text {old }}}{\alpha^{\text {new }}}\left(C^{\text {old }}(x)-C_{0}^{\text {new }}(x)\right)$, $\sigma_{1}, \sigma_{2}$-surface tensions for phases, perpendicular and parallel to concentration gradient. Rather simple algebra, analogous to that in $\mathbf{4 . 1}$, gives

$$
\Delta G=-\alpha \cdot 8 h^{2} r+\gamma(\nabla C)^{2} h^{2} r^{3}+8\left(\sigma_{1} h^{2}+2 \sigma_{2} h r\right),
$$

where

$$
\alpha=n\left(g_{0}^{\text {old }}-g_{0}^{\text {new }}+\frac{\alpha^{\text {old }}\left(C_{0}^{\text {new }}-C_{0}^{\text {old }}\right)^{2}}{2\left(1-\frac{\alpha^{\text {old }}}{\alpha^{\text {new }}}\right)}\right), \quad \gamma=\frac{4 \pi n \alpha^{\text {old }}}{3}\left(1-\frac{\alpha^{\text {old }}}{\alpha^{\text {new }}}\right) .
$$

It is suitable to express $\Delta G$ as a function of volume $V=8 h^{2} r$ and shape parameter $\varphi=\frac{h}{r}\left(r=\frac{1}{2} V^{\frac{1}{3}} \varphi^{-\frac{2}{3}}, h=\frac{1}{2} V^{\frac{1}{3}} \varphi^{\frac{1}{3}}\right)$ :

$$
\Delta G(V, \varphi)=-\alpha V^{1}+\frac{(\nabla C)^{2} \gamma}{32} \varphi^{-\frac{3}{2}} V^{\frac{5}{3}}+2 \sigma_{1}\left(\varphi^{\frac{2}{3}}+2 s \varphi^{-\frac{1}{3}}\right) V^{\frac{2}{3}},
$$

where $s=\sigma_{2} / \sigma_{1}$ is a Wulf parameter.

Function $\Delta G(V \mid \varphi)$ at every fixed volume has one minimum, which is determined by condition $\partial G / \partial \varphi=0$, which gives an optimal shape:

$$
\varphi_{\text {opt }}=\left(\frac{h}{r}\right)_{o p t}=\frac{s}{2}+\sqrt{\frac{s^{2}}{4}+\frac{\gamma(\nabla C)^{2} V}{32 \sigma_{1}}} .
$$

For small volumes ( $V \rightarrow 0$ ), it leads to Wulf rule:

$$
\varphi_{\text {opt }}(V \rightarrow 0)=s=\sigma_{2} / \sigma_{1}
$$

for large volumes, the shape parameter increases as $V^{\frac{1}{2}}$ : 


$$
\varphi_{\text {opt }}(V \rightarrow \infty) \approx\left(\nabla C^{2} \cdot V\right)^{\frac{1}{2}} \cdot\left(\frac{\gamma}{32 \sigma_{1}}\right)^{\frac{1}{2}}
$$

that means the plate-like shape-concentration gradient limits the longitudinal size,

$$
r \underset{V \rightarrow \infty}{\rightarrow} r_{\max }=\left(\frac{4 \sigma_{1}}{\gamma(\nabla C)^{2}}\right)^{\frac{1}{3}} \sim(\nabla C)^{-\frac{2}{3}},
$$

but does not limit the transversal growth,

$$
h \underset{V \rightarrow \infty}{\sim} V^{\frac{1}{3}} \cdot\left(V^{\frac{1}{2}}\right)^{\frac{1}{3}} \sim V^{\frac{1}{2}} \rightarrow \infty .
$$

The shape parameter depends on the product $(\nabla C)^{2} V$ that means some kind of scale invariance. ing:

Thus, the volume dependence of $\Delta G$ for optimized shapes is follow-

$$
\begin{array}{r}
\Delta G(V)=-\alpha V^{1}+\frac{\gamma(\nabla C)^{2}}{32}\left(\frac{s}{2}+\left(\frac{s^{2}}{4}+\frac{\gamma(\nabla C)^{2} V}{32 \sigma_{1}}\right)^{\frac{1}{2}}\right)^{-\frac{4}{3}} V^{\frac{5}{3}}+ \\
+2 \sigma_{1}\left(\left(\frac{s}{2}+\left(\frac{s^{2}}{4}+\frac{\gamma(\nabla C)^{2} V}{32 \sigma_{1}}\right)^{\frac{1}{2}}\right)^{\frac{2}{3}}+2 s\left(\left(\frac{s}{2}+\left(\frac{s^{2}}{4}+\frac{\gamma(\nabla C)^{2} V}{32 \sigma_{1}}\right)^{\frac{1}{2}}\right)^{-\frac{1}{3}}\right)\right) V^{\frac{2}{3}}
\end{array}
$$

One can see that, depending on the value of concentration gradient, $\Delta G$ can be monotonously increasing or nonmonotonic with a maximum (nucleation barrier):
a) $\nabla C>(\nabla C)^{\text {crit }}=\frac{4 \alpha}{3 \sigma_{1}}\left(\frac{2}{3} \frac{\alpha}{\gamma}\right)^{\frac{1}{2}}$-nucleation forbidden,
b) $\nabla C<(\nabla C)^{\text {crit }}$-nucleation possible.

Thus, transversal mode, under condition of shape optimization, gives qualitatively the same results as polymorphic mode:

1) nuclei should be more plate-like, the larger is the volume and the larger is the concentration gradient, shape being determined by they product $(\nabla C)^{2} V$; 
2) nucleation is forbidden if concentration gradient exceeds certain critical value, which can be about $(\nabla C)_{1}^{\text {crit }}=10^{8}-10^{9} \mathrm{~m}^{-1}$.

\subsection{Total Mixing Mode of Nucleation}

Another possibility of nucleation in the fixed gradient is a redistribution of components only inside the forming nucleus resulting in constant concentration $C^{\text {new }}$ and a new lattice, and unchanged gradient outside nucleus. In this case, change of Gibbs free energy due to formation of nucleus is:

$$
\Delta G=4\left(2 \sigma_{1} h^{2}+4 \sigma_{2} h r\right)+n 4 h^{2} \int_{x_{c}-r}^{x_{c}+r}\left(g^{\text {new }}\left(C^{\text {new }}(x)\right)-g^{\text {old }}\left(C^{\text {old }}(x)\right)\right) d x,
$$

Similar to previous subsection one obtains:

$$
\begin{gathered}
\Delta G=4\left(2 \sigma_{1} h^{2}+4 \sigma_{2} h r\right)-\alpha 8 h^{2} r+\gamma_{\|}(\nabla C)^{2} h^{2} r^{3}, \\
\gamma_{\|}=-\frac{4 n}{3} \alpha^{\text {old }} .
\end{gathered}
$$

The main peculiarity here is the negative sign of $\gamma_{\|}$. It means that, contrary to polymorphous and transversal modes, in the case of operating total mixing mode the concentration gradient helps the nucleation. Therefore, at any concentration gradient, a nucleation via total mixing mode is always possible in thermodynamic sense. As we will see below, kinetics can nevertheless suppress such a nucleation. Dependence $\Delta G(\varphi)$ at fixed volumes is nonmonotonic with one minimum and one maximum for small volumes (Fig. 4.8, $a$ ) and monotonously increasing

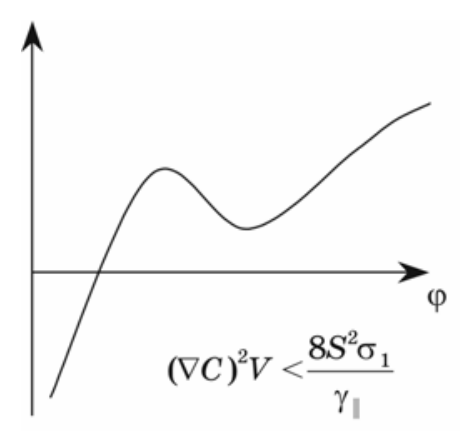

$a$

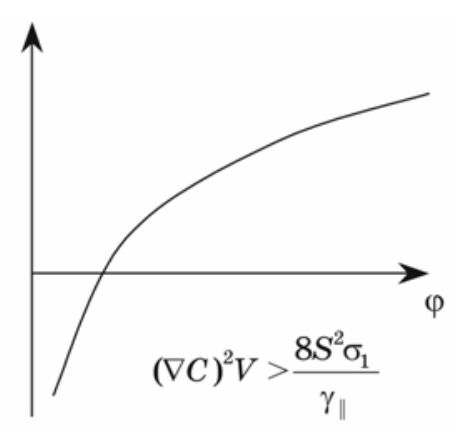

$b$

Fig. 4.8. $\Delta G$ versus shape dependence for total mixing mode at small and large $V(\nabla C)^{2}$. 


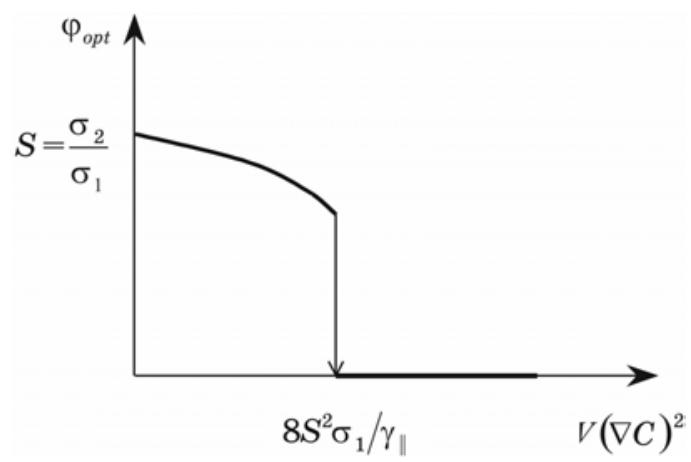

Fig. 4.9. 'Phase shape transition' for total mixing mode.

for large volumes (Fig. 4.8, $b$ ).

Condition of extremum, $\partial G / \partial \varphi=0$, leads to the following equation $\varphi^{2}-S \varphi+\frac{\gamma_{\|}(\nabla C)^{2} V}{32 \sigma_{1}}=0$ with two solutions $\varphi_{1,2}=\frac{S}{2} \pm \sqrt{\frac{S^{2}}{4}-\frac{\gamma_{\|}(\nabla C)^{2} V}{32 \sigma_{1}}}$, first of which corresponds to metastable minimum and second-to maximum.

These solutions disappear, when $(\nabla C)^{2} V>\frac{8 S^{2} \sigma_{1}}{\gamma_{\|}}$, so that nucleus should rapidly transform into needle (see Fig. 4.9). Thus, one has kind of shape phase transition.

Obviously, all above-mentioned considerations are valid only for $\nabla C=$ const, so that needle cannot exceed the size of diffusion zone.

Of course, total mixing mode should operate if the redistribution in transversal direction in the parent phase is absent. For this diffusivity of the new phase should be much larger than the diffusivity of old one. In reality, all nucleation modes operate simultaneously. Description of their interference should be made within the kinetic approach and will be briefly reviewed in the next section.

\section{KINETICS OF NUCLEATION. FOKKER-PLANCK APPROACH}

Kinetics of nucleation is usually described by Fokker-Planck (FP) equation for the size distribution of embryo/nuclei $f(t, N)$, where $f$ is a number of clusters (say, per unit volume) containing $N$ monomers (with size $\left.R \propto(N \Omega)^{1 / 3}\right)$. In FP approach, the clusters are growing or shrinking due to attachment or detachment of individual atoms, so that movement of each cluster in the $N$-space (size space) is quasicontinuous, step-by-step: clusters are not colliding with immediate 
coalescence and not splitting. Therefore, size distribution satisfies the continuity equation in the size space:

$$
\frac{\partial f}{\partial t}=-\frac{\partial j}{\partial N}
$$

where flux $j(N)$ of clusters in the size space contains both stochastic and drift terms:

$$
j=-\bar{v} \frac{\partial f}{\partial N}+\Delta v \cdot f
$$

Stochastic term $-\bar{v} \frac{\partial f}{\partial N}$ is due to randomly attaching and detaching atoms with average frequency $\bar{v}$. Drift term $\Delta v \cdot f$ is determined by thermodynamic profit of attachment comparing with detachment (or vice versa): $\Delta v=v_{+}-v_{-} \propto-\frac{\partial G}{\partial N}$.

We just modified this well-known approach by including the gradient term $\gamma(\nabla C)^{2} N^{5 / 3}$ into Gibbs energy change and taking the time dependence of concentration gradient into account. Detailed derivations can be found in [38]. Here we present the most characteristic figures.

\subsection{Kinetics of the Intermediate Phase Nucleation in the Concentration Gradient: Polymorphic Mode}

Basic kinetic equation for the distribution function $f(N, t)$ for the clusters of new phase:

$$
\frac{\partial f(N, t)}{\partial t}=-\frac{\partial}{\partial N}(\Delta v \cdot f)+\frac{\partial^{2}}{\partial N^{2}}(\bar{v} \cdot f)=-\frac{\partial j}{\partial N},
$$

Here $\bar{v}=\frac{v_{+}+v_{-}}{2}$ is taken to be a constant value,

$$
\Delta v=-\frac{1}{k T} \frac{\partial G}{\partial N} \cdot \bar{v}
$$

In the case of polymorphous mode, the gradient term is positive (concentration gradient hinders the nucleation). Under assumption of spherical nucleus shape, the dependence of Gibbs free energy on the number of atoms in the nucleus has the following form:

$$
\Delta G(N)=\Delta g_{m} \cdot N+\left(\frac{3}{4 \pi n}\right)^{\frac{2}{3}} \frac{g^{\prime \prime}}{10}(\nabla C)^{2} \cdot N^{\frac{5}{3}}+\left(\frac{3}{4 \pi n}\right)^{\frac{2}{3}} 4 \pi \sigma \cdot N^{\frac{2}{3}}
$$


Here $g^{\prime \prime}$ is the difference of second derivatives of Gibbs free energy per atom on concentration between the new phase and parent solution. If the concentration gradient in the nucleation place changes according to parabolic law:

$$
(\nabla C)^{2}=\frac{1}{4 \pi D_{\text {parent }} t},
$$

then a drift term in FP-equation explicitly depends on time, which physically means the lowering of nucleation barrier due to interdiffusion in the parent phase.

It is convenient to use further the non-dimensional variables:

$$
\tau \equiv \bar{v} t, \alpha \equiv \frac{\Delta g_{m}}{k T}<0, \beta \equiv C_{3} \frac{g^{\prime \prime} \frac{\bar{v}}{k T}}{24 \pi D_{\text {parent }}}, \gamma \equiv C_{3} \frac{8}{3} \frac{\pi \sigma}{k T}, C_{3}=\left(\frac{3}{4 \pi n}\right)^{\frac{2}{3}}
$$

Then

$$
\frac{\partial f(N, t)}{\partial \tau}=\frac{\partial^{2} f}{\partial N^{2}}+\frac{\partial}{\partial N}\left(f \cdot\left(\alpha+\beta \frac{N^{\frac{2}{3}}}{\tau}+\frac{\gamma}{N^{\frac{1}{3}}}\right)\right) .
$$

Numeric solution of this last equation has been obtained for the fixed total number of nucleation sites (heterogeneous nucleation):

$$
\int f(N, t) d N=\text { const } .
$$

The evolution in time of the size distribution $f(N)$ corresponds to evolution of the potential field $\Delta G(N)$ but is shifted in time: For small annealing times, when the concentration gradient remains sharp enough, dependence $\Delta G(N)$ is monotonously increasing so that nucleation is thermodynamically forbidden.

During this period, a distribution $f(N)$ remains monotonously decreasing. After certain incubation thermodynamic 'time', when the concentration gradient in the parent phase becomes less than critical value, nucleation becomes thermodynamically possible $(\Delta G(N)$ nonmonotonic with a maximum corresponding to nucleation barrier). Yet, a distribution function $f(N)$ reveals maximum not at once, but after certain 'kinetic incubation period' (Fig. 5.1). We define an incubation time as a period of peak formation for size distribution $f(N)$ (not counting an initial peak at $N=N_{\text {min }}$ ). Obviously, in dimensionless scale $\tau$, the incubation time ( $\tau_{\text {inc }}$ ) should depend on the ratio of two kinetic parameters: $\bar{v}$ and $D_{\text {parent }}$. Dependences $\tau_{\text {inc }}\left(D_{\text {parent }} / \bar{v}\right)$ for different sur- 


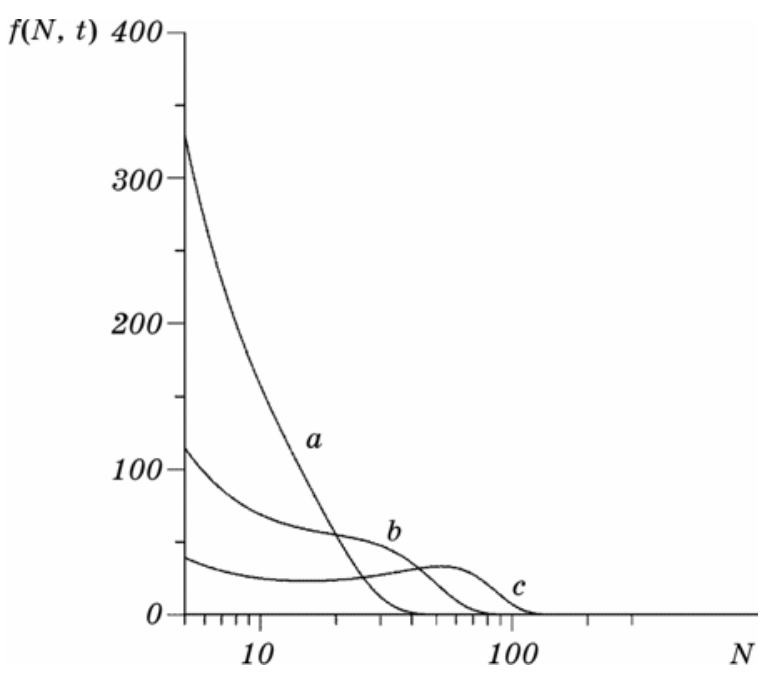

Fig. 5.1. Evolution of size distribution for nuclei of intermediate phase. $\tau=50$ $(a), 100(b), 150(c) . D_{\text {parent }}=10^{-20} \mathrm{~m}^{2} \mathrm{~s}^{-1}, \Delta g_{m}=-7.48 \cdot 10^{-21} \mathrm{~J}, g^{\prime \prime}=\partial^{2} g / \partial C^{2}=$ $=7.77 \cdot 10^{-19} \mathrm{~J}, n=10^{29} \mathrm{~m}^{-3}, T=600 \mathrm{~K}, \sigma=0.15 \mathrm{~J} \cdot \mathrm{m}^{-2}$.

face tensions are shown at Fig. 5.2.

To evaluate the realistic range of the ratio $D / \bar{v}$ one should take into account that the frequency $v$ can be estimated as $v \sim N^{\frac{2}{3}} \cdot D_{\text {boundary }} / \lambda^{2}$, where $D_{\text {boundary }}$ is the diffusivity of interface between new and old

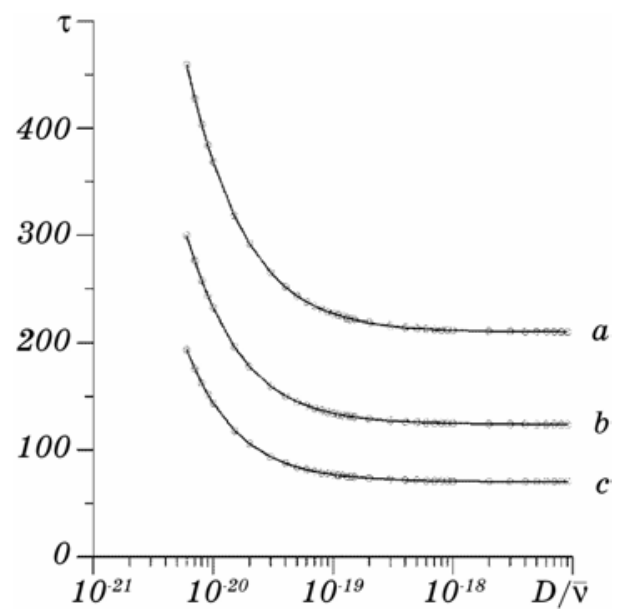

Fig. 5.2. Dependence of dimensionless incubation time $\tau_{\text {inc }}=\bar{v} t_{\text {inc }}$ on the ratio $D / \bar{v}$ for different surface tensions: $0.09(a), 0.10(b)$ and $0.11(b) \mathrm{Jm}^{-2}$. 
phases, and the number of atoms in the nucleus, $\lambda$, is the characteristic length of random walk of atom looking for suitable place of joining the new phase. It is reasonable to suppose that $D_{\text {boundary }}>D_{\text {parent }}$ and $\lambda>a$, where $a$ is an interatomic distance. If $N \sim 30, \lambda \sim 10^{-10}-10^{-8} \mathrm{~m}$, then $D / \bar{v}$ belongs to the interval $10^{-17}-10^{-21} \mathrm{~m}^{2}$.

One can see that with growing diffusivity of parent phase a dimensionless incubation time decreases to some asymptotic level.

It means that this level represents the time for nuclei growth even when gradient effect does not hinder the nucleation. Then we may consider the difference $\tau-\tau_{\min }$ as a time of 'concentration preparation'.

\subsection{Kinetics of Nucleation via Total Mixing Mode}

For total mixing mode, the gradient term (factor $\gamma$ ) in Eq. (5.8) is negative-concentration gradient $\nabla C$ supports the nucleation process. The results of calculation for total mixing mode differ substantially from those for polymorphic mode. First of all, nucleation is never suppressed. Moreover, nucleation behaviour may well be oscillatory; for certain range of parameters, the time evolution of new phase volume is non-monotonic (see Fig. 5.3). Similar non-monotonic behaviour is observed as well for the number of smallest embryos (in our case $N_{\min }=5$ ) for the same thermodynamic parameters except surface tension (Fig. 5.4).

Why are the embryos generated intensively at the very first stage and than partially dissolved? Our answer is following: for total mixing mode, the gradient term supports nucleation. At first, the gradient is

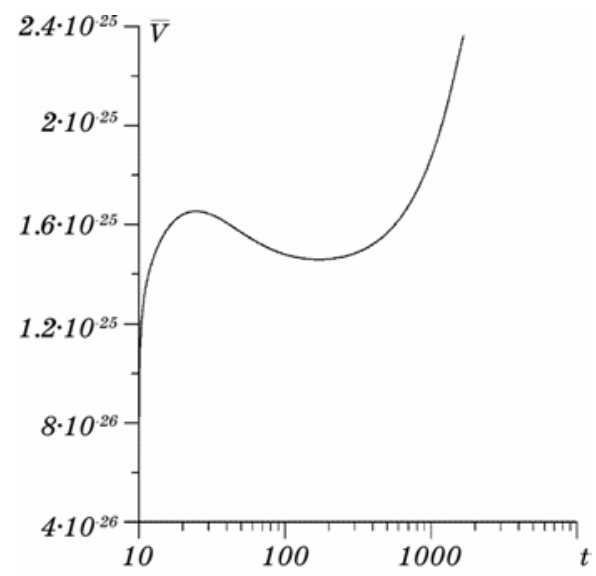

Fig. 5.3. Time dependence of new phase volume in the case of total mixing mode for $\sigma=0.20 \mathrm{Jm}^{-2}$. 


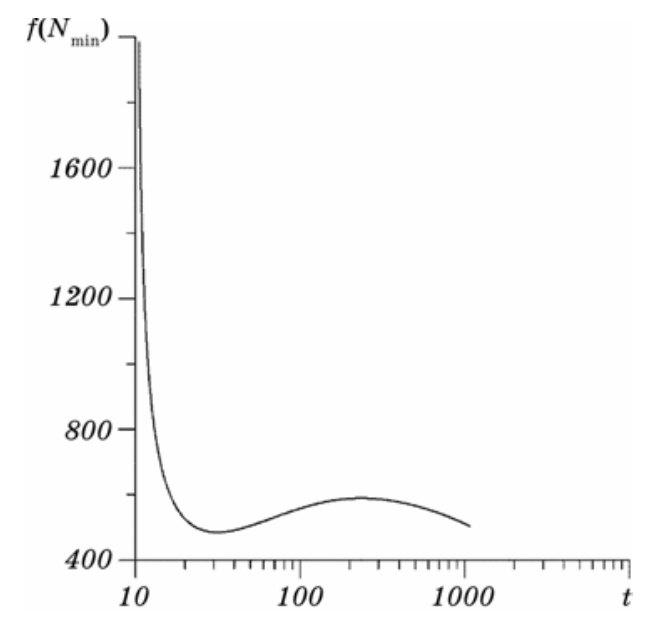

Fig. 5.4. Time dependence of the number of smallest new phase embryos $\left(N_{\min }=5\right)$ for heterogeneous nucleation via total mixing mode for $\sigma=0.15 \mathrm{Jm}^{-2}$.

large making nucleation barrier low and the nucleation process easy. With time the gradient decreases, nucleation barrier and critical nucleus size increase. Therefore, particles which had been generated earlier as overcritical and which did not manage to reach the new critical size, find themselves to be subcritical and are dissolved. In other words, if the growth rate of particles is less than the rate of critical size growth (due to decreasing concentration gradient), these particles will be disintegrated.

We had observed the non-monotonic behaviour of new phase formation during interdiffusion by Monte Carlo simulations both for secondorder and first-order phase transitions in the concentration gradient (Section 2).

\subsection{Interference of Nucleation Modes}

As we have just seen, nucleation of intermediate phase during reactive diffusion can proceed via different 'modes', each being characterized by its own nucleation barrier, own frequency factor and own shape/volume dependence. We restricted ourselves with special shape-parallelepiped, leaving the only free shape parameter $\varphi=h / r$, where $2 h, 2 r$ are the nucleus sizes in transversal and longitudinal directions.

Every mode has its own velocity determined by diffusivities and embryo shape. In [38] we considered the interference of two modestransversal and total mixing. Nucleation modes operate simultaneously with different rates: 


$$
v_{+/-}(N)=v_{+/-}^{(b)}(N)+v_{+/-}^{(c)}(N) .
$$

Rather tricky mathematics using the steady state solutions of FP equation with additive contributions to frequencies from two modes demonstrated that interference leads to dependence of effective nucleation barrier on the ratio of kinetic parameters-the larger is the diffusivity of new phase, the lower is an effective nucleation barrier.

The specific form of the function $\Delta \tilde{G}_{c r}(\tilde{\nabla} c)$ (reduced to nondimensional form) depends on the ratio $\Lambda=\frac{D_{i}}{4 D_{m}} \exp \left(-\frac{\Delta \bar{G}^{(c)}-\Delta \bar{G}^{(b)}}{\theta}\right)$, including the ratio of diffusivities in intermediate and parent phases.

At $\Lambda<\Lambda^{*}$ the function $\Delta \tilde{G}_{c r}(\tilde{\nabla} c)$ is non-monotonous with a maximum (Fig. 5.5), which shifts to the zero $\tilde{\nabla} c$ with increasing $\Lambda$ to $\Lambda^{*}$. With decreasing $\Lambda$ to zero this maximum $\Delta G_{c r}^{\max }$ tends to infinity, (transversal mode is overwhelming). At $\Lambda>\Lambda^{*}$ the function $\Delta \tilde{G}_{c r}(\tilde{\nabla} c)$ is monotonic - the sharper is the concentration gradient, the less is the nucleation barrier (total mixing mode is overwhelming). Thus, strictly speaking, there is no critical gradient in general case, and nucleation of intermediate phase is possible at any gradient due to the possibility of the total mixing mode. Instead of critical gradient beyond which nucleation would be impossible the characteristic gradient exists at which the probability of nucleation is the smallest. In the process of the

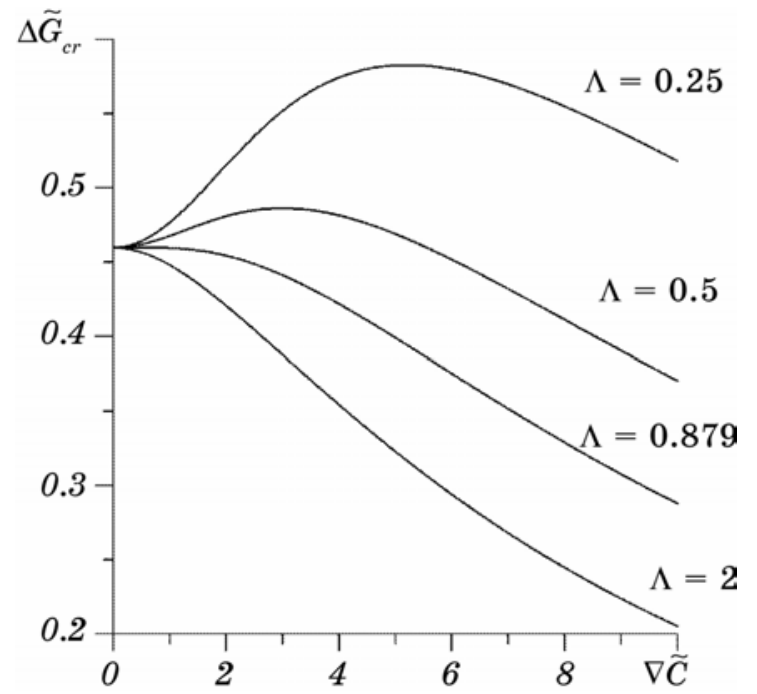

Fig. 5.5. Dependence of an effective nucleation barrier for the critical nucleus on the non-dimensional concentration gradient at different ratios of diffusivities in the new and parent phases. 
interdiffusion in the parent phase, the concentration gradient decreases and the nucleation barrier increases at first. It means that the nuclei of the new phase born at the very beginning can become subcritical at the later stage (if they had no time for growth). Therefore, the oscillatory regime of intermediate phase formation can be possible.

Thus, the kinetic constraints and the interference of different nucleation modes lead to the effective nucleation barriers depending on the ratio of diffusivities as well as on usual thermodynamic factors.

\section{6. 'ALMOST LATERAL' GROWTH AFTER NUCLEATION}

DSC-analysis of reactions in multilayers often shows two peaks of the heat flux corresponding to the same phase. It means that the solidstate reaction of intermediate phase formation proceeds in two stages. According to C. Michaelsen, K. Barmak et al. [14, 67], first stage may correspond to nucleation and lateral growth of new phase islands, and second stage - to their normal layered growth. Direct observations of morphology [67] demonstrate that islands after first stage have rather substantial thickness-about $8 \mathrm{~nm}$ - much more than the critical size. It means that lateral growth proceeds simultaneously with slow normal growth, which is later practically stopped for some period.

First attempts (to our knowledge) of theoretical description of the lateral intermetallic compound growth during peritectoid reaction [68] or simply during reactive diffusion [69] have been based on alternative physical principles but have led to the similar main resultasymptotically constant phase thickness and constant lateral growth velocity. A steady-state model of Klinger et al. [68] treated the curvature gradient as the only driving force of diffusion along the moving interface. The gradient of chemical potential related not to the curvature but to the composition gradient, was neglected (as far as we understand). In our first model [69] we tried to take into account this very facto-gradient of chemical potential along the moving boundary, related to the gradient of composition and leading to a diffusion flux along the interface (as usual for intermetallic compounds, a very small concentration gradient corresponds to a very substantial chemical potential gradient and substantial flux). The concentration gradient corresponds to different supersaturations in different places of the interface. The local velocity of the interface was taken (as usual in the first-order reactions) proportional to the local supersaturation, being maximal at the joint perimeter $A$-IMC- $B$ and minimal at the island tops $A / \mathrm{IMC}$ and $B / \mathrm{IMC}$. The curvature gradient as a driving force of diffusion had been neglected in [69]. This model has led to reasonable asymptotical regimes for thickness and volume but gave not very reasonable shape (shallow) around the top. In [70], we tried to take into account the curvature gradient, as well as nonrectangular wetting an- 
gles but still restricted ourselves with symmetric $A / \mathrm{IMC} / B$ configuration.

Bulk diffusion is assumed to be frozen both in IMC and in parent $A$ and $B$-phases. Only diffusion along the moving interphase boundary is possible. Let at first consider the spherical nucleus of intermediate phase 1 (IMC), appearing at the interface $A / B$. Growth of the new phase particle proceeds due to diffusion of, say, $A$-atoms along the curved interphase boundary IMC/ $B$ with simultaneous reaction of these diffusing atoms with the atoms of $B$-phase. It means that at each moment an $A$-atom migrating along IMC/ $B$ interface has a choice-to migrate further or to react with $B$-atoms, adding a new structural unit of phase IMC and shifting the interface inside phase $B$. Migration rate is governed by interphase diffusivity $D$. The rate of transversal shifting of the interface is taken to be proportional to the difference of the local concentration of $A$ in the boundary and the equilibrium value:

$$
U=k\left(C_{A}-C_{A R}\right),
$$

where $k$ is a reaction constant.

We took an average IMC composition equal to 0.5 , an equilibrium concentrations $C_{A}$ equal to 0.52 ( $A$ /IMC-equilibrium) and 0.48 (IMC/ $B$ equilibrium). The new phase island is treated as a figure of rotation with profile changing with time due to the above-mentioned reaction.

The flux of $A$-atoms is used for reaction and may not reach the top region. It means that the island will be more and more plate-like, normal growth tending to zero.

The main kinetic coefficients are the interface diffusivity $D$ and the reaction constant $k$, the island shape being determined by their ratio.

The initial shape was taken as a sphere of radius $r$. Its cross-section is a circle, transforming into oval-like figure. The length of this circle is divided into elementary intervals, with concentrations changing with time according to the following balance equation:

$$
d C_{A}(i) \delta S(i)=\left(I\left(i+\frac{1}{2}\right)-I\left(i-\frac{1}{2}\right)\right) d t-U(i) S(i)\left(C_{A}(i)-0\right) d t
$$

Here $i$ is a number of cell (Fig. 6.1), $i+\frac{1}{2}$ corresponds to the boundary between $i$-th and $(i+1)$-th cells, $\delta$ is the width of the interface, $S(i)$ is the area of elementary surface generated by rotation of an elementary interval.

$$
S(i)=2 \pi r(i) \sqrt{\left(z\left(i+\frac{1}{2}\right)-z\left(i-\frac{1}{2}\right)\right)^{2}-\left(r\left(i+\frac{1}{2}\right)-r\left(i-\frac{1}{2}\right)\right)^{2}}
$$




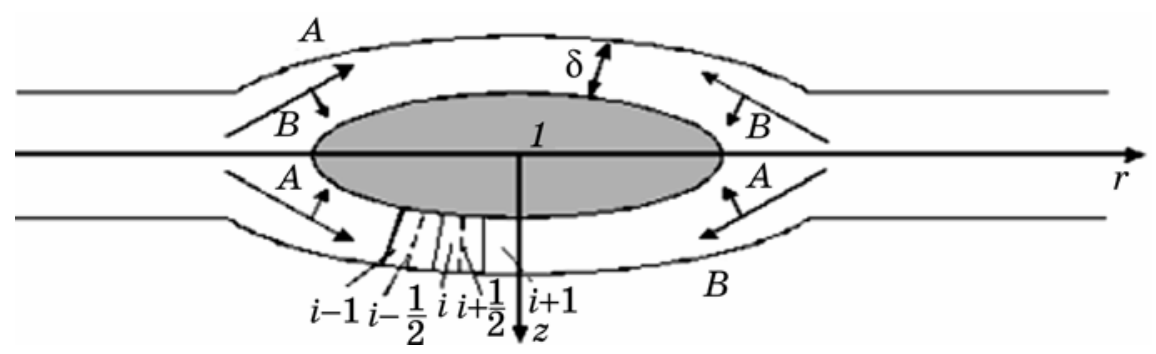

Fig. 6.1. Reaction/diffusion scheme.

The growth of the island proceeds due to the movement of each element in a perpendicular direction with velocity $U(i)=k\left(C_{A}(i)-C_{A R}\right)$. The fluxes are determined by the concentration gradient and by the gradient of curvature:

$$
\begin{gathered}
I_{A}\left(i+\frac{1}{2}\right)=\left(-D \frac{\left[C_{A}(i+1)-C_{A}(i-1)\right]}{\sqrt{(z(i+1)-z(i))^{2}+(r(i+1)-r(i))^{2}}}-\right. \\
-\frac{\frac{\sigma_{1 B} \Omega}{k_{b} T}\left[C_{A}(i+1)+C_{A}(i-1)\right]\left[\frac{1}{R(i+1)}-\frac{1}{R(i-1)}\right]}{\sqrt{(z(i+1)-z(i))^{2}+(r(i+1)-r(i))^{2}}} \delta 2 \pi r(i),
\end{gathered}
$$

where $R$ is the local radius of curvature. Of course, the movement of interval boundaries with different velocities changes the lengths of all intervals. Therefore, we redefine the equidistant grid at each new time step, using interpolation procedure.

Boundary conditions are introduced in the following way: at each time step, the marginal interval $(i=0)$ moves along the normal direction, 'breaking link' with the $A / B$ interface. Simultaneously the new point of grid is added at the $A / B$ interface $(z(0)=0$ ), at the distance $\frac{U(0) d t}{\sin \theta_{1 B}}$, where wetting angles are determined by Young's relation $\sigma_{A 1} \cos \theta_{1 A}+\sigma_{1 B} \cos \theta_{1 B}=\sigma_{A B}$. After this, the grid is redefined by interpolation procedure restoring the number of intervals. To simplify our scheme, we considered only the symmetric case $\theta_{1 A}=\theta_{1 B}$. The main results of calculations are as follows:

the island thickness tends to some asymptotical value $z_{\max }$;

the thickness $z_{\max }$ and the time derivative $d z / d t$ decreases with in- 

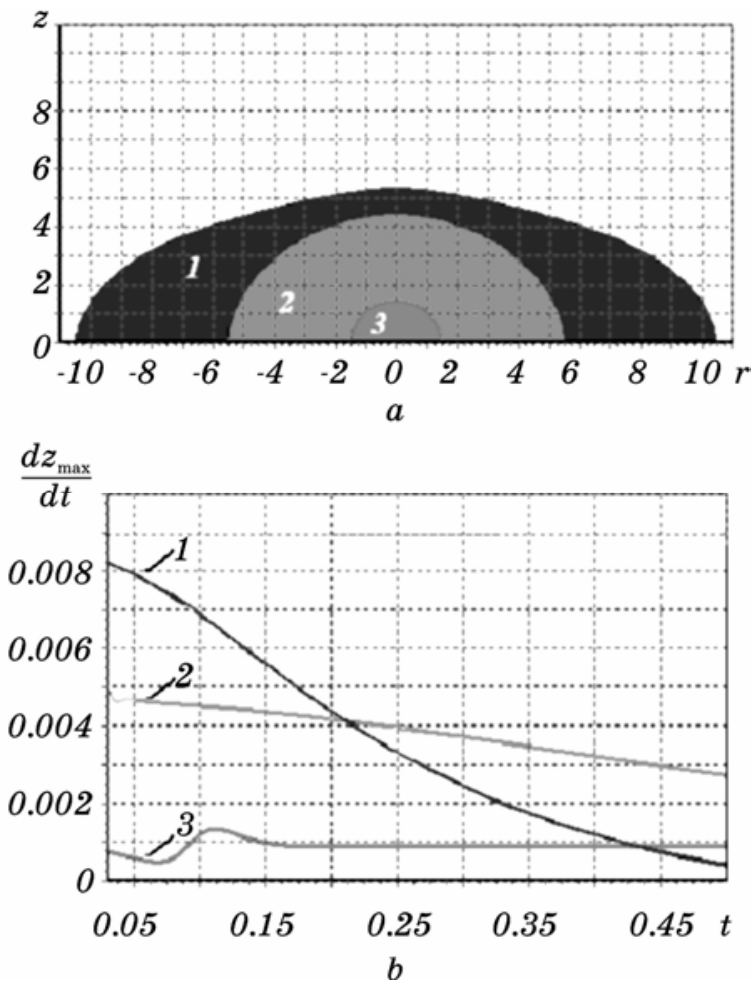

Fig. 6.2. Shapes $(a)$ and time dependences $(b)$ of the thickening growth velocity $d z / d t$ for a diffusivity $D=1 \cdot 10^{-15} \mathrm{~m}^{2} / \mathrm{s}$ and different reaction rate constants: (1) $k=1 \cdot 10^{-6} \mathrm{~m} / \mathrm{s} ;$ (2) $k=5 \cdot 10^{-7} \mathrm{~m} / \mathrm{s} ;$ (3) $k=1 \cdot 10^{-7} \mathrm{~m} / \mathrm{s}$. All $\sigma=0.3 \mathrm{~J} / \mathrm{m}^{2}$.

creasing reaction rate $k$ (Fig. 6.2) and increases with increasing diffusivity $D$ along the moving interface;

the aspect ratio (shape factor) $z_{\max } / r(0)$ decreases with increasing reaction rate constant and decreasing diffusivity.

\section{NUCLEATION IN NANOPARTICLES AND COMPETITIVE NUCLEATION IN BULK MATERIALS}

Previous sections treated the case of intermediate phase (or several phases) formation during reactive diffusion. Now let us consider simpler situation, when the intermediate phase(s) appears as a result of decomposition in supersaturated binary alloy. Two 'nano-cases' can be distinguished: (1) nucleation in the array of separated nanoparticles and (2) simultaneous nucleation of many clusters in the bulk material, when each nucleation site has limited nanovolume of the 'responsibil- 
ity region' (further also as supply region). In the first case, the existence of external boundary of the particle may influence the nucleation process. In the second case, the ripening effect can be important at some stage. Yet, both cases have something in common-it is a limited amount of material for construction of the new phase (in nanoparticle, it is limited forever, in bulk material it is limited till/if the intensive ripening starts).

The effect of depletion in the parent phase on nucleation and growth at a nanometre scale cannot be neglected. In the following, we briefly review the thermodynamic study of nucleation and growth in confined volumes, considering this effect.

We consider the decomposition of a supersaturated binary alloy $\alpha$ (containing $A$ and $B$ components) leading to the formation (when it is not suppressed) of two-phase system $\alpha+1$ or $\alpha+2$, where 1, 2 are intermediate phases which both have non-zero driving forces of transformation. In all cases, depletion of the nanosized parent phase increases the Gibbs free energy of the system and makes nucleation more difficult. Thus, the decrease of the size $R$ (particle size or half-distance between nucleation sites), should increase the nucleation barrier.

Moreover, when two phases can nucleate, they will compete for atoms the amount of which is limited (in the following, the competition will concern $B$ atoms). Result of competition depends on $R$, interface energies $\sigma$, driving forces (per atom) $\Delta g_{1}, \Delta g_{2}$, initial composition $C_{0}$ (mole fraction of $B$-specie), compositions of the new phases $C_{\text {new }}$ (then also as $X_{1}$ or $X_{2}$ ) and temperature $T$.

First, the thermodynamics associated with the formation of a single intermediate phase in a nanometric volume is treated.

\subsection{Nucleation/Suppression Criterion for Single Intermediate Phase in a Small Volume}

Very simplified illustration of the essence of the size effect related to parent phase depletion can be following. If the nucleus of the new phase 1 of radius $r^{*}$ with mole fraction $C_{\text {new }}$ of specie $B$ is formed in a parent phase with much less initial mole fraction $C_{0}$, it should 'suck out' the atoms $B$ from the sphere (supply region) with radius not less than $R^{*}=\left(\left(C_{\text {new }} v_{1}\right) /\left(C_{0} v\right)\right)^{1 / 3} r *$ (here $v$ and $v_{1}$ are the volume per atom in the parent and new phases respectively). Obviously, if the size of whole particle is less than mentioned value $R$, and $r *$ is the critical size of nucleus in bulk material, nucleation becomes impossible-total number of atoms $B$ in the whole particle is just not enough for construction even of single stable nucleus. Taking $r^{*}=10^{-9} \mathrm{~m}, C_{0}=0.1$ and $v / v_{1} \approx 1, C_{\text {new }}=0.8$, one obtains for the 'minimal' radius $R^{*}$ of the supply region $R^{*}=2 \mathrm{~nm}$. When $R<2 \mathrm{~nm}$, nucleation is absolutely impossible. Actually, this is only estimation, since with depletion of par- 
ent phase with, say, atoms $B$, the Gibbs free energy increases due to decrease of entropy. Therefore, nucleation becomes impossible already for some particle size $R>R^{*}$. To obtain quantitative results, we should choose the thermodynamic models for the new and parent phases.

At any moment and at any size $r$ (radius) of growing nucleus, mole fraction distribution is supposed to be step-like (without transient layers) so that concentration is uniform inside each phase. Analogous scheme, for single intermediate phase, has been proposed [71] within the framework of Cahn-Hilliard approach [72] with gradient terms and diffuse interface.

It is suggested that for any given nucleus size $r$, the mole fractions $C_{\text {new }}$ and $C_{0}+\Delta C$ in the new and parent phases respectively satisfy the condition of minimum for the Gibbs free energy. The following conservation law relates the mole fractions:

$$
C_{o} V_{o}=C_{n e w} V_{n}+\left(C_{o}+\Delta C\right) V_{\alpha} .
$$

In relation 1, the number of atoms per unit volume $n$ is taken the same for all phases. For spherical clusters inside spherical particles:

$$
V_{n}=\frac{4}{3} \pi r^{3}, \quad V_{o}=\frac{4}{3} \pi R^{3}, \quad V_{\alpha}=V_{o}-V_{n} .
$$

Thus, mole fraction $C_{\text {new }}$ is a function of depletion $\Delta C$. Conditions $\frac{\partial \Delta G}{\partial \Delta C}=0, \frac{\partial^{2} \Delta G}{\partial \Delta C^{2}}>0$ determine an optimal depletion for fixed nucleus size.

The driving force for transformation per particle or per one nucleation site is:

$$
\Delta G=n\left(V_{n} g_{\text {new }}\left(C_{\text {new }}\right)+\left(V_{o}-V_{n}\right) g_{\text {old }}\left(C_{o}+\Delta C\right)-V_{o} g_{\text {old }}\left(C_{o}\right)\right)+\sigma S .
$$

$C_{\text {new }}$ is related to $C_{0}$ as $C_{\text {new }}=C_{o}+\left(\frac{R^{3}}{r^{3}}-1\right)(-\Delta C) ; S=4 \pi r^{2}$.

Once more, we use the parabolic approximations:

$$
\begin{gathered}
\Delta g_{\text {new }}(C)=\Delta g_{\text {new }}\left(C_{n}^{0}\right)+\frac{\alpha_{\text {new }}}{2}\left(C-C_{n}^{0}\right)^{2}, \\
\Delta g_{\text {old }}(C)=\Delta g_{\text {old }}\left(C_{o}^{0}\right)+\frac{\alpha_{\text {old }}}{2}\left(C-C_{o}^{0}\right)^{2} .
\end{gathered}
$$

Condition $\left(\frac{\partial \Delta G}{\partial \Delta C}\right)_{r, R}=0$ coincides with the classical parallel tangents 
rule but here depletion of the parent phase is taken into account. It gives:

$$
\Delta C^{\text {opt }}=-\frac{C_{n}^{o}+\frac{\alpha_{\text {old }}}{\alpha_{\text {new }}}\left(C_{o}-C_{o}^{0}\right)-C_{o}}{\frac{R^{3}}{r^{3}}-1+\frac{\alpha_{\text {old }}}{\alpha_{\text {new }}}} ; \quad C_{\text {new }}^{\text {opt }}=\left(\frac{R^{3}}{r^{3}}-1\right)\left(-\Delta C^{\text {opt }}\right)+C_{o} .
$$

Substituting $\Delta C^{o p t}(r, R)$ and $C_{\text {new }}^{o p t}(r, R)$ into expression (7.2) for $\Delta G$, one obtains $\Delta G$ as a function of one 'reaction coordinate' $r$ (at fixed $R$ ).

$\Delta G(r, R)$ has been calculated the following values for the parameters: $n=6 \cdot 10^{28} \mathrm{~m}^{-3}, C_{o}=0.32, C_{n}{ }^{0}=0.8, C_{o}{ }^{0}=0.2, \Delta g\left(C_{n}{ }^{0}\right)-\Delta g\left(C_{o}{ }^{0}\right)=0, \alpha_{\text {new }}=\alpha_{\text {old }}=$ $=10^{-19} \mathrm{~J}$ and $\sigma=0.15 \mathrm{Jm}^{-2}$. Results are represented in Fig. 7.1.

For small $R=2.3 \mathrm{~nm}$ (situation III), $\Delta G(r)$ is monotonically increasing which means that nucleation is impossible.

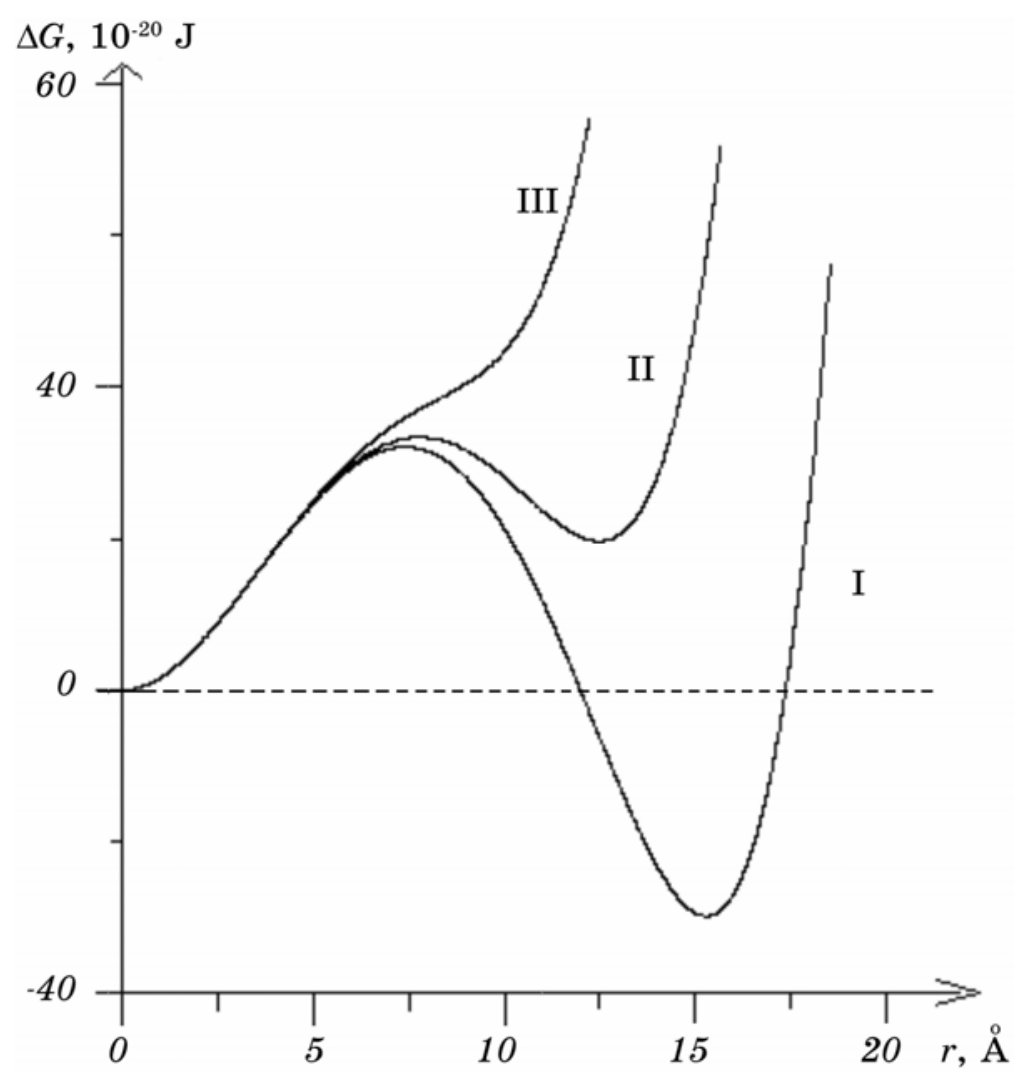

Fig. 7.1. Dependences $\Delta G(r, R)$ on nuclei radius $r$ and external particle size $R$ as a parameter. I-decomposition $\left(R_{\mathrm{I}}=32 \cdot 10^{-10} \mathrm{~m}\right)$, II-metastable $\alpha+1$ state $\left(R_{\mathrm{II}}=28 \cdot 10^{-10} \mathrm{~m}\right)$, III-nucleation forbidden $\left(R_{\mathrm{III}}=23 \cdot 10^{-10} \mathrm{~m}\right)$. 
For $R=2.8 \mathrm{~nm}$ (situation II), a function $\Delta G(r)$ has a metastable minimum laying for higher Gibbs energy than the initial state.

For large $R(R=3.2 \mathrm{~nm}$, situation I in Fig. 7.1), $\Delta G(r)$ presents the classical form with one maximum defining the nucleation barrier $\Delta G(r *)$ and a minimum corresponding to final decomposition.

Situations I and II, in the case of multiple simultaneous nucleation at nucleation sites, can be compared to the so called 'mob effect': 'If people pass the door one by one, they all do succeed, but if they try to do it simultaneously, the process stops'.

Thus, it has been shown from thermodynamics that nucleation in nanometric volumes in very small single particles as well as when multiple simultaneous nucleation with short distance between the nucleation sites occurs, nucleation can be suppressed due to depletion of the parent phase.

The previous analysis demonstrates that the effect of depletion in the parent phase on nucleation and growth at a nanometre scale cannot be neglected. The following thermodynamic study of nucleation and growth in confined volumes is based on such an effect and will be represented in the frame of phase diagram method (Section 7.2).

Furthermore when two phases compete for nucleation and growth the following regimes had been found to be possible, depending on supply region size (or particle size) and thermodynamic parameters of parent and intermediate phases: (1) total prohibition of separation for both phases, (2) formation and stabilization of metastable phase instead of stable one, (3) relative stabilization of metastable phase with the temporary delay of its transformation into the stable phase after certain crossover point (in the case of sufficiently high additional barrier for next transformation), (4) formation and growth of stable phase, when the metastable phase does not appear at all, (5) formation and growth of stable phase via the metastable phase (Section 7.3).

\subsection{Phase-Diagram Method of Analysis for Nanosystem}

Let us choose the model of the new phase as a 'line' (strictly stoichiometric) intermediate phase and exclude the elastic contributions into the Gibbs energy. We will consider formation of the spherical nucleus of intermediate phase inside the spherical particle of supersaturated solid solution at initial concentration $X_{0}$ (Fig. 7.2).

The Gibbs energy per atom of the new phase 1 (line compound) and of the parent phase are taken as (Fig. 7.3):

$$
\begin{gathered}
\Delta g_{1}(T)=\Delta g_{1}+\alpha k T \quad\left(X=X_{1}\right), \\
\Delta g_{o}(T, X)=k T\{X \ln (X)+(1-X) \ln (1-X)\} .
\end{gathered}
$$



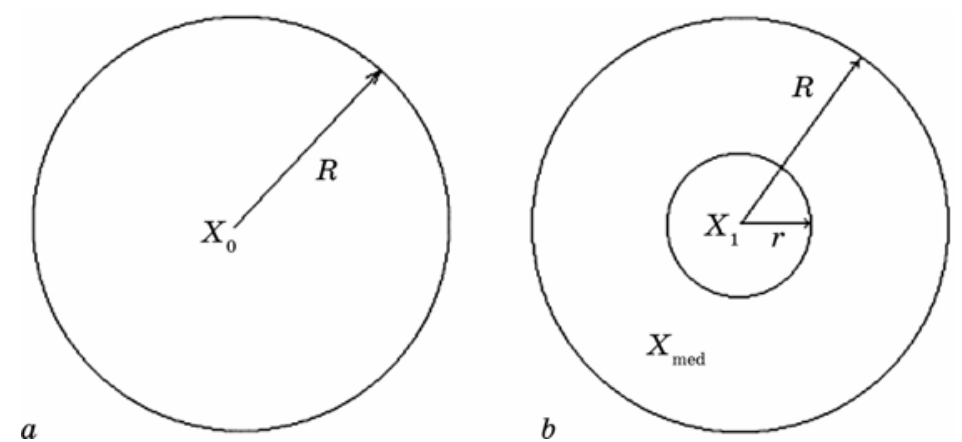

Fig. 7.2. Schematic representation of phase transformation: $(a)$-particle of concentration $X_{0}$ before transformation, $(b)$ - the same particle after the transformation with concentration redistribution being taken into account: $X_{\text {med }}(r)$-concentration of ambient parent phase, $X_{1}$-concentration of strong stoichiometric intermediate phase, $r$-nucleus size, $R$-radius of supply region (nanometric isolated particle).

Hereby, $\Delta g_{1}$ is the isothermal Gibbs energies of formation (per atom) from pure solid components, $\alpha>0$-non-dimensional parameter de-

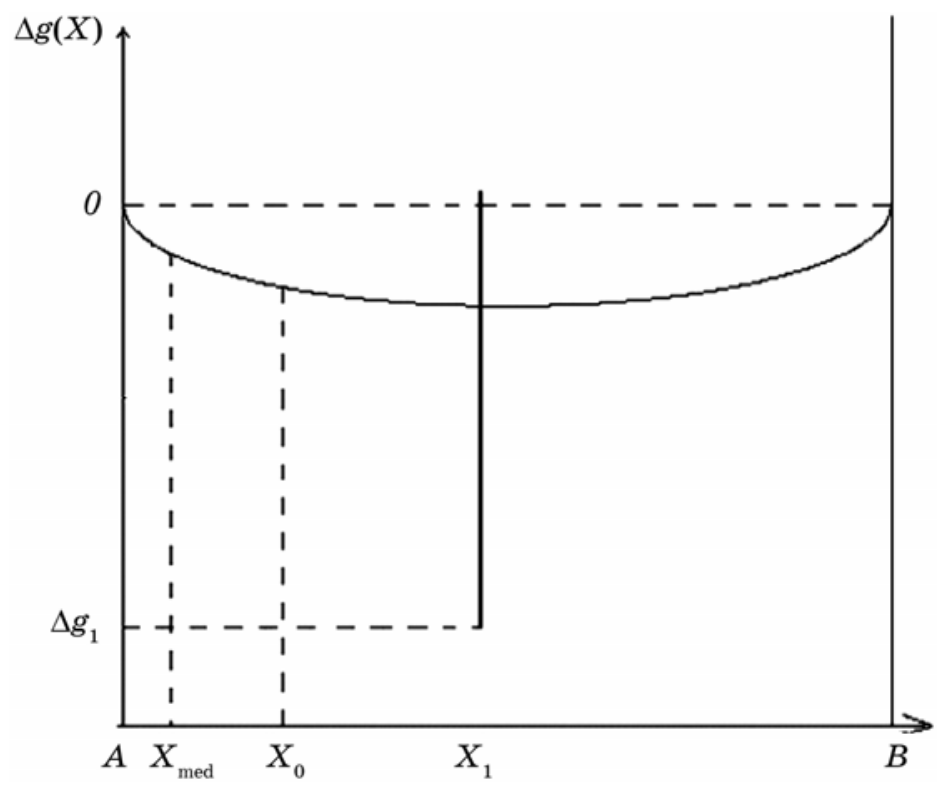

Fig. 7.3. $\Delta g(X)$ - Gibbs free energy (per atom) as a function of composition for 'old' (parent) and 'new' (phase 1) phases (qualitative dependence). $X_{0}$ is the initial composition of the parent phase, $X_{\text {med }}(r)$-composition of the parent phase as a result of fluctuation/nucleation and/or separation. (In this diagram, the pure components are supposed to have the same structure.) 
termining temperature-dependent behaviour of driving forces, $k$ is the Boltzmann constant.

The expression for the driving force is:

$$
\Delta G=n V_{1} \Delta g_{1}+n\left(V-V_{1}\right) \Delta g_{\text {old }}\left(X_{m e d}\right)-n V \Delta g_{\text {old }}\left(X_{0}\right)+4 \pi r^{2} \sigma
$$

In Eq. (7.8), the number of atoms per unit volume $n$ is taken the same in the two phases ( $\left.V_{1}=\frac{4}{3} \pi r^{3}, V_{1}=\frac{4}{3} \pi R^{3}\right)$.

Extremes of $\Delta G$ function in Eq. (7.8) have been found by direct calculation of $\Delta G$ for all reasonable sizes $r$ (with small step).

Let us plot the Gibbs free energy versus nucleus size $\Delta G(r)$ according to the Eq. (7.8) for different values of temperature, initial concentration $X_{0}$ or size of particle. The typical schematic dependences are presented in Fig. 7.4.

One can obtain spectrum of all states by changing the initial supersaturation (due to composition and temperature), size of transforming particle. Namely, possible cases are separation (cases $T_{3}, R_{3}, X_{03}$ in Fig. 7.4), metastable state (cases $T_{2}, R_{2}, X_{02}$ in Fig. 7.4), and impossibility of separation (cases $T_{1}, R_{1}, X_{01}$ in Fig. 7.4). The last situation for small particle may be realized even at concentrations and temperatures for which separation is possible in infinite alloy.

Let us choose a criterion of separation for parent phase in small isolated particle and of stability of new two-phase equilibrium. Under separation criterion, we will understand the situation at which the

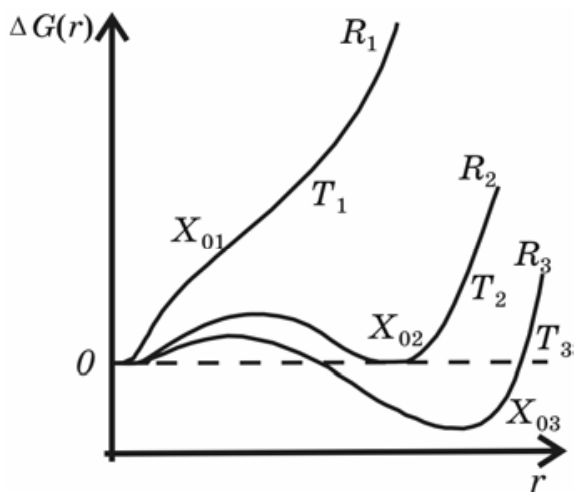

Fig. 7.4. Schematic Gibbs free energy dependence $\Delta G$ on radius of nucleus $r$ : - for different temperatures $T_{1}>T_{2}>T_{3}$ and fixed other parameters; - - - for different initial concentrations $X_{0}\left(X_{01}<X_{02}<X_{03}\right)$, provided other parameters are fixed; - - for different radii of supply region $R_{1}<R_{2}<R_{3}$ respectively. Graphs $T_{2}, R_{2}$ and $X_{02}$ (middles) correspond to the separation criterion. 
Gibbs free energy dependence on radius of a new phase becomes nonmonotonic with maximum and zero second minimum: $\Delta G=0$, $\left.\frac{\partial \Delta G}{\partial r}\right|_{R}=0$ for $r>0$ (cases $T_{2}, R_{2}, X_{02}$ in Fig. 7.4). In the case of small particle the equilibrium condition means the equal depths of two pits (in Gibbs free energy dependence on radius $r$ ), which are separated by thermodynamic barrier. In the case of small particle, this barrier is of nucleation barrier order and may be not high $(<50 k T)$. It means that the phase equilibrium in ensemble of small particles will correspond to statistic distribution, at which one part of particles will be in singlephase state and second part-in two-phase state.

Let us take the size of our binary system: $R=10^{-8} \mathrm{~m}$ (at same fixed parameters: $n=7 \cdot 10^{28} \mathrm{~m}^{-3}, \sigma=0.15 \mathrm{~J} \cdot \mathrm{m}^{-2}, \Delta g_{1}=-3 \cdot 10^{-20} \mathrm{~J}, X_{1}=0.5, \alpha=$ $=2.4$ ). According to the separation criterion for small particle (case $X_{02}$ in Fig. 7.4) one can find the optimal composition $X_{\text {med }}^{\text {opt }}$ ( $X_{\text {med }}^{\text {opt }} \equiv X_{\text {med }}^{\text {opt }}(R, T)$ ) of the parent phase corresponding to stable $\Delta G(r)$ minimum. Thus, we have two important points (third one, $X_{1}=0.5$, is determined from initial condition) for chosen criterion: initial composition as the limit solubility $X_{0}^{c r}(R, T)$ (further $X_{0}^{c r}$ ) of one component in another ( $B$ in $A$ ) and optimal composition of the depleted ambient parent phase $X_{\text {med }}^{\text {opt }}$ as the result of separation. Size dependent separation diagram is presented in Fig. 7.5 (case $M Q Z N L$ ).

Notice that in contrast to analysis for cupola-shaped separation diagram of massive alloy one needs to interpret the size dependent separation diagram for small particle otherwise. This is clear from following reasons. In fact, the usual cupola-shaped equilibrium diagram determines solubility $X_{0}^{c r}$ as well as equilibrium compositions $\left(X_{m e d, \infty}=X_{0}^{c r}\right.$ and $\left.X_{1}\right)$ as a result of separation by one line ( $\left.A S H P N F\right)$.

For small particle, the equilibrium diagram becomes doubled (and shifted and size dependent). That is, instead of one line we distinguish two lines, namely, line $M Q Z N L$ of solubility $X_{0}^{c r}$ and line $D Q E N L$ of separation results: $X_{\text {med }}^{\text {opt }}$ and $X_{1}$. Hence, the limiting solubility in small particle does not coincide with the equilibrium composition after the separation. We call this effect 'critical supersaturation' [72-73]. It means that separation is possible only (at some fixed temperature and size) if $\Delta X>\Delta X^{c r}$. Here $\Delta X^{c r}=X_{0}^{c r}-X_{m e d}^{\text {opt }}$ is the 'critical supersaturation', that is the difference between the limiting mean mole fraction of component $B$ in initially saturated alloy (or solubility-concentration corresponding to separation criterion) and optimal (or equilibrium) concentration in parent phase after the separation. If the supersaturation $\Delta X$ is less than the 'critical supersaturation' $\left(\Delta X<\Delta X^{c r}\right)$, which is the certain value for an alloy of fixed size $R$ and of temperature $T$, then nucleation and separation are impossible. The physical reason of such a peculiarity consists of two factors. First one is a conservation law ef- 


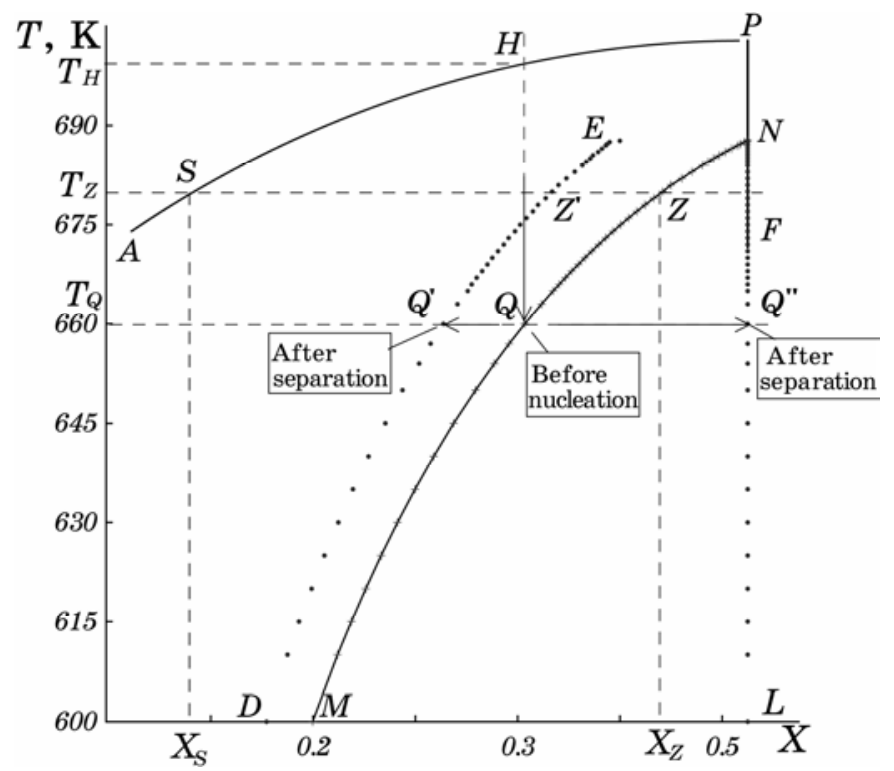

Fig. 7.5. Size dependent state diagram 'temperature-composition'. Continuous line $A S H P N F$-cupola-shaped diagram of binary system for the case of separation in infinite system, which is found analytically when the new phase is strictly stoichiometric; $M Q Z N L$-cupola-shaped diagram of small particle at fixed radius $R$ (line connecting the experimental points '+' is plotted for visualization of cupola-shape); set of points $D Q E N L$ determines the result of separation: $X_{\text {med }}^{\text {opt }}$ (points $D Q E$ ) and $X_{1}=0.5$ (points $N L$ ) in small particle. The parameters are in the main text.

fect and the second one is that the separation in small particle may start only from nucleus formation, volume of which is not small with respect to the total system volume.

Thus, in description of separation of supersaturated solution in small particle one must distinguish the solubility limit (maximal concentration of impurity before separation) and equilibrium concentration of depleted parent phase after separation-difference being called a 'critical supersaturation'.

For finite rate of $T, R$ or $X_{0}$ changes, one should observe hysteresis behaviour. Contrary to usual hysteresis, which vanishes at infinitely slow processes $\left(\frac{d T}{d t} \rightarrow 0\right.$ or $\frac{d R}{d t} \rightarrow 0$, or $\left.\frac{d X_{0}}{d t} \rightarrow 0\right)$ the critical supersaturation does not disappear; being some thermodynamic characteristics depending on $T$ and $R$. Increasing (decreasing) the size the magnitude of critical supersaturation $\Delta X^{c r}$ decreases (increases). In limiting case of infinite environment, the 'critical supersaturation' $\Delta X^{c r}$ becomes zero. 


\subsection{Competitive Nucleation and Growth of Two Intermediate Phases}

As shown in the previous section, limited volumes of the decomposing alloy can change both the rate and the very result of decomposition. If two phases have the driving force to nucleate, the competition between them is inevitable, first of all, competition for necessary constructing material. One can expect interesting possibilities if phase 2 has both a smaller driving force of transformation and interface energy $\left(\sigma_{20}<\sigma_{10}\right)$. In principle, different possibilities must be considered. They are represented in Fig. 7.6.

The analysis of competitive nucleation is presented in detail in [53].

To catch the main idea, let the intermediate phases be line compounds with fixed compositions $X_{1}, X_{2}$, and the parent supersaturated phase behaves like an ideal solution (see Fig. 7.7).

The Gibbs energy per atom of the parent supersaturated phase is presented in Eq. (7.7). The expression for the driving force is:

$$
\begin{aligned}
\Delta G=n V_{1} \Delta g_{1}+ & n V_{2} \Delta g_{2}+n\left(V-V_{1}-V_{2}\right) \Delta g_{\text {old }}\left(X_{\text {med }}\right)- \\
& -n V \Delta g_{\text {old }}\left(X_{0}\right)+\Delta G_{\text {surf }} .
\end{aligned}
$$

Hereby, $\Delta g_{2}$ and $\Delta g_{1}$ are the isothermal Gibbs energies of formation

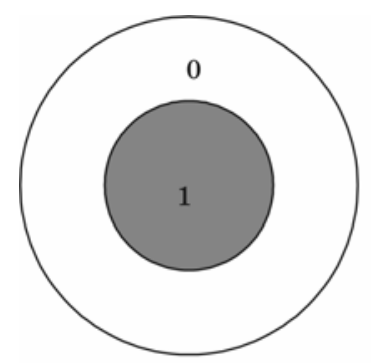

$a$

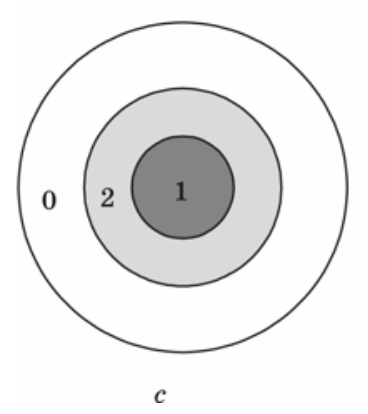

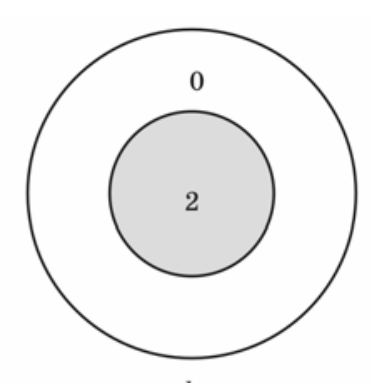

$b$

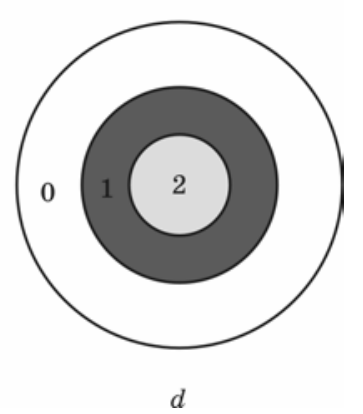

Fig. 7.6. Modes of nucleation and growth of new phases in the centre of a small particle or a region of supply: $(a)$-nucleation of phase $1 ;(b)$-nucleation of phase $2 ;(c),(d)$-competitive formation of phase 1 and phase 2 . 


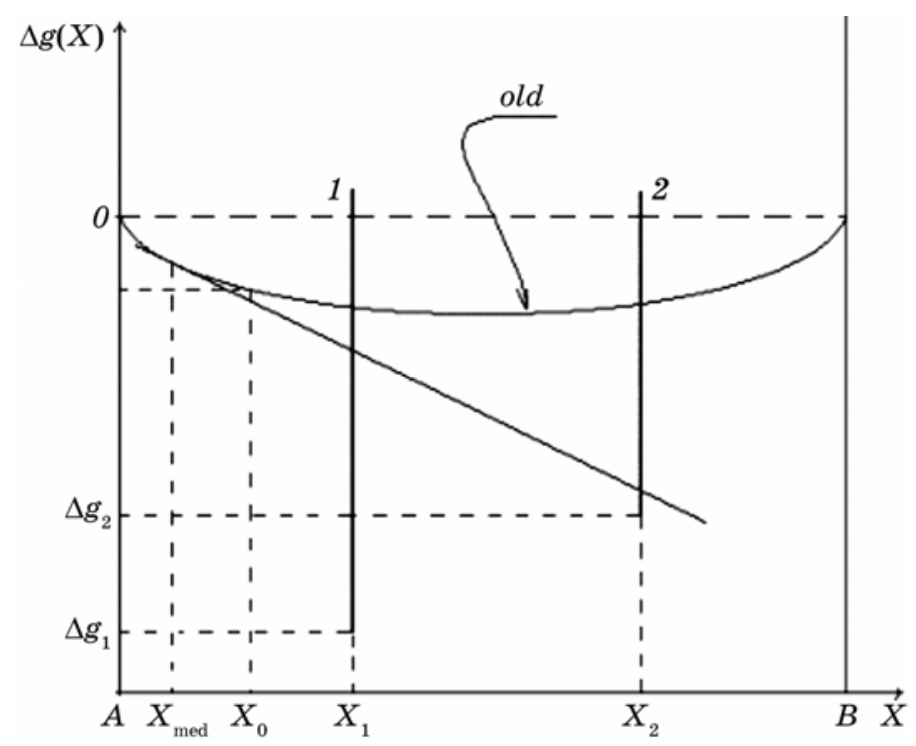

Fig. 7.7. $\Delta g(X)$ - Gibbs free energy (per atom) as a function of composition for 'old' and 'new' phases. $X_{0}$ is the initial composition of the parent phase. (In this diagram, the components are implicitly supposed to have the same structure). Hereby, $\Delta g_{1}$ and $\Delta g_{2}$ are the isothermal Gibbs energies of formation (per atom) from pure solid components.

(per atom) from pure solid components.

The conservation law leads to:

$$
X_{o} V=X_{1} V_{1}+X_{2} V_{2}+X_{m e d}\left(V-V_{1}-V_{2}\right),
$$

where again the number of atoms per unit volume $n$ is taken the same in the three phases.

The reaction co-ordinate $X_{\text {med }}$ (medium composition) decreases from $X_{0}$ in the process of evolution. $V=\frac{4}{3} \pi R^{3}$ is the total volume of particle or of the 'supply region'.

According to the configurations represented in Fig. 7.6, it follows:

(a) $V_{1}=\frac{4}{3} \pi r_{1}^{3}, \quad V_{2}=0, \quad \Delta G_{\text {surf }}=4 \pi r_{1}^{2} \sigma_{10}$,

(b) $V_{1}=0, \quad V_{2}=\frac{4}{3} \pi r_{2}^{3}, \quad \Delta G_{\text {surf }}=4 \pi r_{2}^{2} \sigma_{20}$,

(c) $V_{1}=\frac{4}{3} \pi r_{1}^{3}, \quad V_{2}=\frac{4}{3} \pi\left(r_{2}^{3}-r_{1}^{3}\right), \quad \Delta G_{\text {surf }}=4 \pi r_{12} \sigma_{12}+4 \pi r_{2}^{2} \sigma_{20}$, 
(d) $V_{1}=\frac{4}{3} \pi\left(r_{1}^{3}-r_{2}^{3}\right), \quad V_{2}=\frac{4}{3} \pi r_{2}^{3}, \Delta G_{\text {surf }}=4 \pi r_{2}^{2} \sigma_{21}+4 \pi r_{1}^{2} \sigma_{10}$.

State parameters $r_{1}, r_{2}, X_{\text {med }}$ are linked by conservation law (7.10). Therefore, $\Delta G$ can be treated as a function of two independent parameters $\Delta G\left(r_{1}, X_{\text {med }}\right)$ or $\Delta G\left(r_{2}, X_{\text {med }}\right)$. Numeric analysis shows that minimisation of $\Delta G$ at fixed $X_{\text {med }}$ gives $r_{1}=0$ or $r_{2}=0$. It means that the threephase configurations $\alpha+1+2$ (cases $(c)$ and $(d)$ in Fig. 7.6) appeared to be less favourable than two-phase configurations $\alpha+1$ (case $(a)$ in Fig. 7.6) or $\alpha+2$ (case (b) in Fig. 7.6). Therefore, dependences of $\Delta G\left(X_{\text {med }}\right)$ are presented only for regimes (case $(a)$ in Fig. 7.6) and (case $(b)$ in Fig. 7.6). Results can be gathered into the following three cases:

Case 1. When the interface energies are such that $\sigma_{10}<\sigma_{20}$ with bulk driving force for phase 1 larger than for phase 2; phase 1 has a lower nucleation barrier and its formation is more favourable in general. Then system will stop in absolute minimum state (decomposition $\alpha+$ 1). That is the usual case of total suppression of the metastable phase 2 by stable phase 1 .

Case 2. When $\sigma_{20}<<\sigma_{10}$ with bulk driving force for phase 1 larger than for phase 2 ; nucleation of phase 1 is more difficult.

It appears that phase 2 will nucleate first (suppressing the formation of phase 1 ) and the system will stop in state $(\alpha+2)$. In this case, a small volume helps phase 2 to suppress the precipitation of phase 1 . Moreover, it is noteworthy that the absolute minimum of Gibbs energy for phase 2 is being reached during the evolution of reaction coordinate before the correspondent value for phase 1 .

All cases including impossibility of phase 1 formation (owing to too small volumes) even in the absence of phase 2 must also be considered. Actually, they have been considered above for the case of nucleation of single phase.

Case 3 or crossover regime. When the conditions $\sigma_{20}<\sigma_{10}$ with a driving force for phase 1 larger than for phase 2 are satisfied, the following other situation can be set up: Nucleation remains easier for phase 2 but with a minimum of Gibbs energy for phase 1 being deeper than for phase 2 but this for a greater depletion (smaller $X_{m e d}$ ). Then, a crossover from regime (a) to regime (b) becomes possible (see Fig.).

At first stage of the evolution path of the system, phase 2 should nucleate and grow, since nucleation barrier for phase 2 is smaller than for phase 1. But beyond the crossover-point, phase 1 becomes more favourable, so that further depletion beyond this point favours transformation $\alpha+2 \rightarrow \alpha^{\prime}+1$. However, this transformation (second stage of evolution) obviously means one more Gibbs energy barrier $\Delta G_{12}$ due to the required formation of the interface between phase 1 and phase 2 . The 'kinetic decoding' of this additional transformation at the crossover point will be more fully discussed in a future work. Here, only general considerations are given about this point. 
If this additional barrier $\Delta G_{12}$ is small enough, so that the sum of $\Delta G_{12}$ and nucleation barrier for phase 2 will be less than nucleation barrier for phase 1 , then the evolution path of the formation of the phase 1 via phase 2 becomes more favourable and phase 2 helps to form phase 1 .

On the contrary, sum of $\Delta G_{12}$ and value of nucleation barrier for phase 2 will be larger than nucleation barrier for phase 1 ( $\Delta G_{12}$ becomes sufficiently high to hinder the transformation). In this case, the system can reach the metastable state and is able to remain there for a long time.

It is of interest to study, through a practical example, whether a metastable state, at the sample level, can be attained owing to a very high nucleation frequency and how such a metastable state may compete with the formation of the stable phase. Authors of [53] studied a practical example of $\mathrm{Al}-\mathrm{Li}$ alloy. The possibility of formation and total stabilization of metastable $\mathrm{Al}_{3} \mathrm{Li}_{1}$ phase instead of stable $\mathrm{Al}_{1} \mathrm{Li}_{1}$ phase in small particles of $\mathrm{Al}-(5-50)$ at. $\% \mathrm{Li}$ alloys had been demonstrated.

\section{MODEL OF DISCONTINUOUS PRECIPITATION BASED ON THE BALANCE AND MAXIMUM PRODUCTION OF THE ENTROPY}

In previous section, we considered a decomposition process provided by bulk diffusion in nanoparticles or nanoalloys. At low temperatures, when bulk diffusion is frozen, nature finds other ways of reducing its Gibbs energy. Usually such reduction is provided by easy-path diffusion via moving grain-boundaries (discontinuous precipitation and DIGM) or interphase boundaries (Section 6). A model of discontinuous precipitation in supercooled binary polycrystalline alloys at reduced temperatures, taking place as a result of the diffusion-induced grainboundary migration, is constructed with allowance of grain-boundary diffusion [57]. The proposed approach allows independent determination of the main parameters, including the interplate distance, the maximum velocity of the phase transformation front, and the concentration jump at this boundary. This is achieved by using a set of equations for the (i) mass transfer in the moving interphase boundary, (ii) balance of the entropy fluxes at the phase transformation front, and (iii) maximum rate of the free energy release. The model uses minimum thermodynamic information about the two-phase system: the curvature of the Gibbs potential surface in the decomposing phase and the free energy of the interface between the new phases.

Figure 8.1 shows the configuration of a model system representing a binary alloy $\alpha_{0}$ decomposing with the formation of a single cell of the depleted phase $\alpha$ in equilibrium with phase $\beta$. The transformation region $R$ moving at a constant velocity $v$ toward the decomposing phase $\alpha_{0}$ coincides with the boundary between $\alpha_{0}$ and $\alpha$ phases and has the height $b$, width $h$, and length $\Delta z$. Let $c^{\alpha / \beta}$ denote the equilibrium con- 

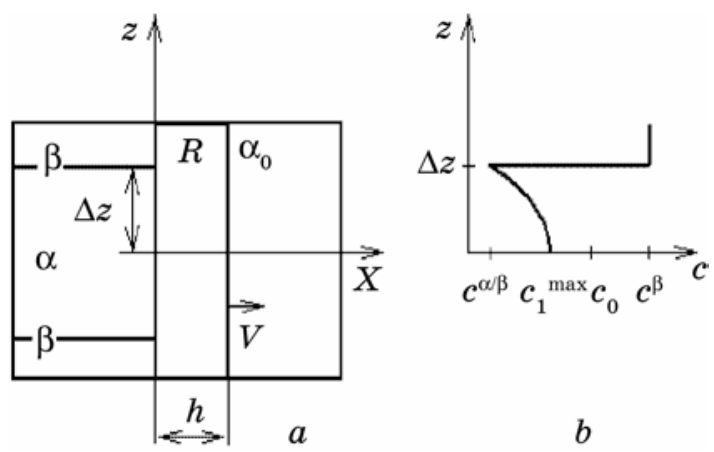

Fig. 8.1. A model system for discontinuous precipitation: $(a)$-cross-section in $x-z$ plane; $(b)$-concentration distribution behind the transformation front at the concentration $c_{0}$ of the supersaturated solid solution.

centration at the $\alpha-\beta$ interface in the $\alpha$ phase; $c^{\beta}$, the equilibrium concentration in the $\beta$ phase plate; and $c_{1}^{\max }$, the maximum concentration in the $\alpha$ phase (at the centre of the $\alpha$ phase plate along the $x$ axis).

The thermodynamic stimulus of the discontinuous precipitation, as well as of any other process at a constant temperature and pressure, is a decrease in the Gibbs potential. A model phase diagram in Fig. 8.2 illustrates this. The dominating factor is a change in the chemical energy, while the role of elastic energy is insignificant. The motion of the transformation front partly decreases the degree of non-equilibrium in the system by reducing the concentration in the $\alpha$ phase cell and by inducing the $\beta$ phase separation with the $\alpha-\beta$ interface formation. Attaining the perfect equilibrium behind the transformation front corresponds to the formation of $\alpha$ phase cells with the $c^{\alpha / \beta}$ concentration, which is principally possible in the case of a strongly retarded phase boundary.

At low temperatures, the rate of discontinuous precipitation is controlled by the diffusion of components in the flat grain boundary mov-

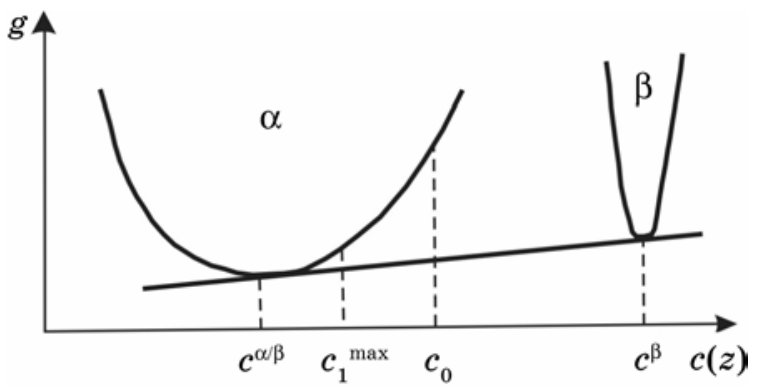

Fig. 8.2. A model phase diagram and phase equilibria. 
ing at a constant velocity $v$ (for the frozen bulk diffusion) [6, 74]. The dependence of concentration in the $\alpha$ phase on the coordinate $z$ along the transformation boundary is described by a quasi-stationary grainboundary diffusion equation for a moving boundary [74] based on the balance of component fluxes (without specification of the mechanism of atomic jumps):

$$
s \tilde{D} \frac{d^{2} c(z)}{d z^{2}}+\frac{c_{0}-c(z)}{h} v=0
$$

Here, $s$ is the segregation coefficient, $\tilde{D}$ is the coefficient of mutual diffusion in the phase boundary $R, c(z)$ is the local concentration in the boundary $R$, and $h$ is the boundary width. The Cahn solution to this equation is as follows:

$$
c(z)=c_{0}-\left(c_{0}-c^{\alpha / \beta}\right) \frac{\operatorname{ch}(z / L)}{\operatorname{ch}(\Delta z / L)},
$$

where the co-ordinate $z$ varies from zero (in the middle of the $\alpha$ phase plate) to $\Delta z$ at the $\alpha-\beta$ interface. Accordingly, $c(z)$ changes from $c_{1}^{\max }$ to $c^{\alpha / \beta}$ over a half-length of the cell, depending on the combination of parameters $\Delta z / L$. The interplate distance $\Delta z$, the kinetic coefficient $L=\sqrt{s \tilde{D} h / v}$, and the triple product $s \tilde{D} h$ are related as

$$
\operatorname{sh} \tilde{D}=\frac{1}{C} \Delta z^{2} v
$$

where $C$ is the Cahn parameter determined from the relation $\frac{c_{0}-c_{1}}{c_{0}-c^{\alpha / \beta}}=\frac{2}{\sqrt{C}} \operatorname{th}\left(\frac{\sqrt{C}}{2}\right)$, and $c_{1}$ is the average concentration in the $\alpha$ phase.

Each kinetically possible solution for an arbitrary transformation rate and the corresponding value of the parameter $L$ gives a certain interplate distance $\Delta z$. For $\Delta z / L \rightarrow 0$, the maximum concentration $c_{1}^{\max }$ in the $\alpha$ phase (at the centre of the $\alpha$ phase plate and along the plate) tends to the equilibrium value $c^{\alpha / \beta}$. As the $\Delta z / L$ ratio increases, the degree of redistribution of the components decreases, the interphase boundary velocity increases, and the system remains substantially non-equilibrium. Thus, a single equation (8.1) with the solution (8.2) cannot unambiguously describe the kinetics of the discontinuous precipitation and do not provide for the independent determination of $\Delta z$ and $L$. In order to eliminate this uncertainty, Cahn [74] employed the principle of maximum decrease in the free energy $\Delta F$ during the discontinuous precipitation reaction. This was achieved by considering a linear relationship between $\Delta F$ and the boundary velocity and by intro- 
ducing the second kinetic coefficient representing the boundary mobility [74]. However, this linear relationship is not always confirmed in experiment [75].

The main idea of the approach developed in [57] consists in a more thorough analysis of the balance of energy release and degradation in the transformation boundary moving at a constant velocity. In contrast to the Cahn model, it is suggested that the total decrease in the free energy has to be balanced with all ways of the energy degradation: both via diffusion along the transformation region and via interphase $\left(\alpha_{0}-\alpha\right)$ jumps of atoms (with the jumping kinetics determining mobility of the phase boundary). For independently determining the main kinetic parameters, we use the principle of maximum rate of the free energy release [76], the equation of balance of the entropy fluxes, and the Cahn solution (8.2) of the mass transfer equation. The analysis is performed for a diffusion-controlled discontinuous precipitation reaction under the assumption of a readily moving (high-mobility) interphase boundary.

Let us assume that a change in the entropy $d_{e} S$ during the elementary time interval $d t$ as a result of the phase transformation in the region $R$ (coinciding with the interphase boundary) shifting by its width is equal to the change in the entropy $d_{i} S$ as a result of the diffusion redistribution of components in the same region $R$. We consider a quasistationary process obeying the condition [77]

$$
\frac{d S}{d t}=\frac{d_{i} S}{d t}+\frac{d_{e} S}{d t}=0,
$$

which means that the total entropy change in the moving open system is zero (this condition is valid at a constant transformation front velocity $v$ ). In order to pass from the rate of entropy change to the rate of free energy release, we can use the relation (valid at a constant temperature and pressure)

$$
\frac{d S_{i, e}}{d t}=-\frac{1}{T} \frac{d G_{i, e}}{d t} .
$$

In this case, the rate of the free energy release is [77, 78]

$$
\Psi \equiv T \frac{d_{i} S}{d t}=T \int_{V} \sigma d V=-\frac{d_{i} G}{d t}=\frac{d_{e} G}{d t}>0 .
$$

The entropy production as a result of the diffusion redistribution of components in the region $R$ can be written as [77-79]

$$
\Psi=\frac{h b}{\Delta z} \int_{0}^{\Delta z} I X d z=\frac{h b}{\Delta z} \int_{0}^{\Delta z}(-s \tilde{D}) \frac{\partial c(z)}{\partial z}\left(-\frac{\partial \tilde{\mu}(z)}{\partial z}\right) d z,
$$


where $I$ is the generalized flux along the $z$-axis, $X$ the driving force, and $\tilde{\mu}(z)=\mu_{A}-\mu_{B}=\partial g / \partial c_{A}$ is the generalized chemical potential at a point $z$ of the region $R$.

Let us determine a change in the Gibbs potential as a result of the transformation of the $\alpha_{0}$ phase in an element $d z+d z^{\prime}$ at the point $z$ of the interphase boundary. For this element, transformed into a band of the $\alpha$ phase of length $d z$ and a band of the $\beta$ phase of length $d z^{\prime}$, the law of conservation of the substance yields

$$
c_{0}\left(d z+d z^{\prime}\right)=c(z) d z+c^{\beta} d z^{\prime} .
$$

The change of the Gibbs potential be written as

$$
\Delta G(z)=g(c(z)) d z+g^{\beta} d z^{\prime}-g\left(c_{0}\right)\left(d z+d z^{\prime}\right),
$$

where $g(c(z)) d z$ is the Gibbs potential of the $\alpha$ phase band of length $d z$ at the point $z$ with the concentration $c(z)$ in the region $R, g^{\beta}$ is the Gibbs potential of the $\beta$ phase, and $g\left(c_{0}\right)$ is the Gibbs potential in the supersaturated $\alpha_{0}$ phase in front of the transformation boundary. Expanding the Gibbs potential for the $\alpha$ and $\alpha_{0}$ phases into the Taylor series with respect to $g\left(c^{\alpha / \beta}\right)$ and retaining terms up to the second order (which is permissible for regular solutions with a large energy of mixing), we obtain

$$
\Delta G=\left.\frac{1}{2}\left[\left(c(z)-c^{\alpha / \beta}\right)^{2}-\frac{c^{\beta}-c(z)}{c^{\beta}-c_{0}}\left(c_{0}-c^{\alpha / \beta}\right)^{2}\right] g^{\prime \prime}\right|_{c^{\alpha / \beta}}=f(c(z)) k,
$$

where $f(c(z))<0$ and $k=\left.g^{\prime \prime}\right|_{c^{\alpha / \beta}}>0$ is the curvature of the Gibbs potential surface in the $\alpha$ phase, at the point with concentration $c^{\alpha / \beta}$.

The driving force can be expressed as

$$
X=-\frac{\partial \tilde{\mu}}{\partial z}=-\frac{\partial c(z)}{\partial z} k
$$

where

$$
\tilde{\mu}=\frac{\partial \Delta G}{\partial c(z)}=\left[\left(c(z)-c^{\alpha / \beta}\right)-\frac{\left(c_{0}-c^{\alpha / \beta}\right)^{2}}{2\left(c^{\beta}-c_{0}\right)}\right] k .
$$

The rate of the free energy release as a result of the phase transformation in the region $R$ can be written as

$$
\Psi=\frac{d_{e} G}{d t}=-\frac{v b}{\Delta z} \int_{0}^{\Delta z} \Delta G(c(z)) d z-g^{\alpha / \beta} b v,
$$


Here, the first term in the right-hand part is the energy gain per unit volume and the second term corresponds to the formation of a new boundary region of length $h$ between $\alpha_{0}$, and $\beta$ phases. The Gibbs potential per unit volume is $g^{\alpha / \beta}=2 \gamma / \Delta z$, where $\gamma$ is the free energy per unit area of the $\alpha-\beta$ interface.

Using expressions (8.2), (8.4), (8.5), (8.8)-(8.10) and integrating with respect to $z$, we eventually obtain

$$
\begin{gathered}
\Psi=\frac{d_{e} G}{d t}=-\frac{k L v}{16 \Delta z}\left(c_{0}-c^{\alpha / \beta}\right)^{2} \times \\
\times\left\{\frac{\Delta z}{L} \operatorname{sch}^{2}\left(\frac{\Delta z}{L}\right)-\frac{3 c^{\beta}-c_{0}-2 c^{\alpha / \beta}}{\left(c^{\beta}-c_{0}\right)} \operatorname{th}\left(\frac{\Delta z}{L}\right)\right\}- \\
-\frac{2 \gamma v}{\Delta z}=-\frac{d_{i} G}{d t}=\frac{k L v}{2 \Delta z}\left(c_{0}-c^{\alpha / \beta}\right)^{2}\left\{\operatorname{th}\left(\frac{\Delta z}{L}\right)-\frac{\Delta z}{L} \operatorname{sch}^{2}\left(\frac{\Delta z}{L}\right)\right\} .
\end{gathered}
$$

This equation determines the first relation between $\Delta z$ and $L$ :

$$
k L\left(c_{0}-c^{\alpha / \beta}\right)^{2}=8 \gamma\left\{\frac{\Delta z}{L} \operatorname{sch}^{2}\left(\frac{\Delta z}{L}\right)-\frac{2 c^{\alpha / \beta}-c_{0}-c^{\beta}}{c^{\beta}-c_{0}} \operatorname{th}\left(\frac{\Delta z}{L}\right)\right\}^{-1} .
$$

Once this relationship is established, we can use the principle of maximum rate of the free energy release for determining the optimum $\Delta z$ from the condition

$$
\frac{d \Psi}{d(\Delta z)}=0 .
$$

The explicit expression (8.13) is very complicated. For this reason, let us use a numerical method for finding the maximum of expression (8.13) with respect to $\Delta z$ under the condition (8.12) (see Fig. 8.3). As a result, we obtain a solution to the system of equations (8.12) and (8.13) by determining the values of $\Delta z=\Delta z^{S O L}$ and $L$ for the given values of the triple product $\operatorname{sh} \tilde{D}$ and the equilibrium concentration $c^{\alpha / \beta}$. For the comparison with experiment, we will use the data for Ni-1.4 at.\% For system at $703 \mathrm{~K}$ [6] with the following model parameters: $k=1.075 \cdot 10^{11} \mathrm{~J} / \mathrm{m}^{3}, \gamma=0.5 \mathrm{~J} / \mathrm{m}^{2}, c_{0}=1.4$ at. $\%, c^{\alpha / \beta}=0.43$ at. $\%$, $c^{\beta}=25$ at. $\%$, and $s \tilde{D} h=2.12 \cdot 10^{-24} \mathrm{~m}^{3} / \mathrm{s}$, the calculations yield the values of parameters coinciding with the experimental values: the average concentration in the cell, $c_{1}=0.82$ at. $\%$; the interplate distance, $\Delta z^{\mathrm{SOL}}=0.134 \cdot 10^{-6} \mathrm{~m}$; and the Cahn parameter, $C=9.2$. The transformation boundary velocity is determined by the formula $v=s \tilde{D} h / L^{2}=2 \cdot 7 \cdot 10^{-10} \mathrm{~m} / \mathrm{s}$. For the given parameters, a change in the Gibbs free energy determined by integrating relation (8.8) and taking into account the surface energy gives $\Delta G=-14.6 \mathrm{~J} / \mathrm{mol}$, which coin- 


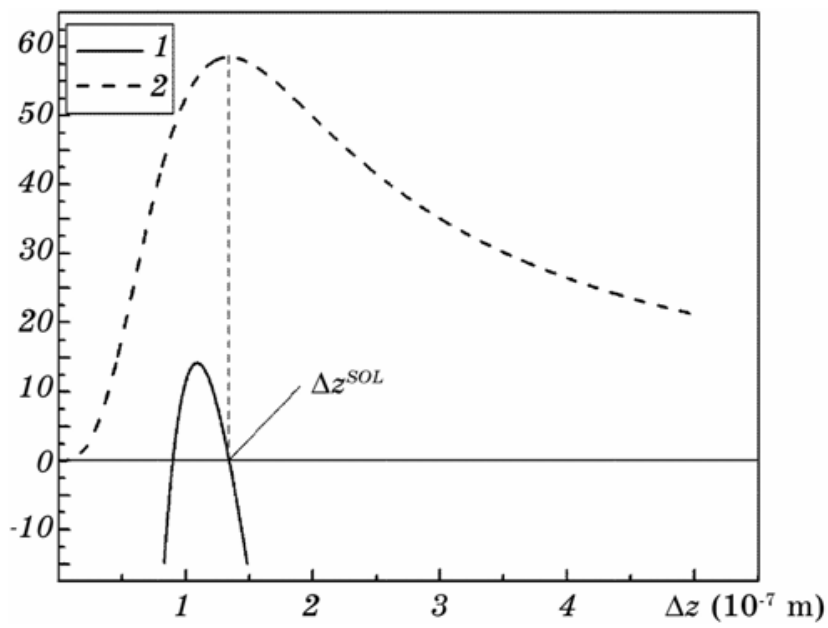

Fig. 8.3. Calculated: (1) dependences of the difference between LHS and RHS of Eq. (8.12) on interlammelar semiperiod; (2) rate of free energy release in transformation region. The optimal solution corresponds to $\Delta z^{S O L}$.

cides with the experimental value.

Thus, using the equation of balance of the entropy fluxes at the phase transformation front, we obtained a new scheme for determining the main parameters of cellular decomposition without using the Cahn assumption concerning a linear relationship between the driving force and the transformation front velocity (the form of this relationship can be established in the course of calculations). The proposed scheme allows additional ways of the energy degradation in the transformation region to be taken into account, in particular, those related to a reduced mobility of the interphase boundary or to the presence of driving forces of various kinds.

\section{NANOSTRUCTURE FORMATION DURING THE SHOCK LOADING AND ANOMALOUS MASS-TRANSFER}

Rather unexpected area of possible applications of nano-ideas is a mass transfer proceeding in metals under pulse loading with deformation rates $\dot{\varepsilon} \sim 1-10^{6} \mathrm{~s}^{-1}[58,59,80-83]$. Numerous experiments (with tracers as well as with standard diffusion couples) show that such a fast deformation leads to mass transfer with huge penetration depth of $10^{-6}-10^{-4} \mathrm{~m}$ at very small loading times $\tau \sim \varepsilon_{\max } / \dot{\varepsilon} \sim 10^{0}-10^{-6} \mathrm{~s}$ (maximal deformation $\varepsilon_{\max }$ is typically about $10-15 \%$ ). In most cases, the concentration profiles for tracers or impurity atoms have approximately Gaussian form, $c(x) \approx c(0) \exp \left(-\alpha x^{2}\right)$, which allows introducing (at least formally) an effective diffusivity $D=(4 \alpha \tau)^{-1}$, which 
can well be about $10^{-5}-10^{-7} \mathrm{~m}^{2} / \mathrm{s}$ (much more than in liquids). Thus, effective diffusivities are huge. What mechanism can explain such migration rates?

The first idea was 'mechanical diffusion' [61], when stochastic plastic shifts make atoms to demonstrate random walk (each step is a shift with different blocks of matter). Authors of [58] claim, that it is not the case. Their arguments are following. In case of mechanical diffusion, the effective 'diffusivity' of single atoms and molecules should be the same. Yet, special experiments with some salt, containing tracer atoms, deposited on the surface prior to loading, demonstrated (according to authors) that molecules remain practically immovable. We will discuss other possible interpretation of this experiment in the concluding paragraph of this section.

Enhanced diffusion under pulse loading cannot be attributed to vacancies or to dislocations since increasing density of vacancies or of dislocations leads to decrease of effective diffusivity [58, 83]. Grain boundaries are also bad candidates for explanation, since in the vicinity of GBs the concentration of tracers drops [58, 59, 83].

Several authors proposed to explain anomalous mass transfer as a result of diffusion with drift, but this approach is unable to explain, for example the following fact: if tracers are situated between two samples, after loading they are redistributed in both directions almost symmetrically.

We tried to investigate at least some aspects of above-mentioned phenomena by means of molecular dynamics (MD), applied to modelling of shock wave propagation in $\mathrm{Cu}$.

\subsection{Model}

1. System consisted of $6 \times 6 \times 42$ elementary f.c.c. cells (6048 atoms) with periodic boundary conditions along axes perpendicular to shock direction.

2. Before shock started, atoms had been situated in the equilibrium sites, and their initial velocities were distributed according to Maxwell with certain 'starting' temperature.

3 . Prior to shock initiating the system relaxed, kinetic and potential energy were redistributed, and the final mean temperature was established. If the mean temperature should be equal to the starting one, the velocities were renormalized.

4. Shock was generated by motion of two boundary atomic planes with constant velocity $U$ along the $<001>$-direction.

5 . Motion equations have been solved according to modified Verlet's algorithm.

6. Interactions have been modelled by the pair Born-Mayer potential and, alternatively, by many-body Sutton-Chen potential. 
7. Special block of the program made possible the return of interstitial atoms into vacant sites, generation of new interstitials and vacancies, generation of dumbbell configurations.

\subsection{Results}

To check the validity of our model, we measured the dependence of the shock wave velocity $V_{S W}$ on the shock velocity $U$ in the interval $U \in$ $(1000,3000) \mathrm{m} / \mathrm{s}$.

Thickness of SW front was only 3-4 atomic planes, which is reasonable since no dislocations were present. According to experimental data for copper [6],

$$
V_{\text {SW }} \cong a+b U \text {, }
$$

where $a_{\text {exper }}=3958 \mathrm{~m} / \mathrm{s}, b_{\text {exper }}=1.5$. Our simulations gave $a_{\text {exper }}=3905$ $\mathrm{m} / \mathrm{s}, b_{\text {exper }}=1.5268$.

Density, temperature and pressure were determined as average values for each atomic plane. Temperature was displayed in the coarsened space scale (averaged over 5 planes).

Pressures, ratio of densities and temperature behind the SW front are given in Table 9.1. Pressures and densities practically coincide with experimental data. Temperatures are much higher than in real experiments (usually $\Delta T$ is not more than $100 \mathrm{~K}$ [58]).

\section{Behaviour of the Point Defects}

In absence of the shock wave, the main mechanism for diffusion of interstitial defects appeared to be 'kick-out'. Propagation of SW front for any shock velocity $U$ did not lead to capturing of defect atom for long distances. Behaviour of interstitial atom was a random process and different runs gave different results: a) capturing of defect atom

TABLE 9.1. Dependence of pressure, density and temperature behind SWfront on the shock velocity $U$.

\begin{tabular}{r|r|c|c|r}
\hline$U[\mathrm{~m} / \mathrm{s}]$ & $\Delta p_{\text {model }}[\mathrm{GPa}]$ & $\Delta p_{\text {theor }}[\mathrm{GPa}]$ & $\xi=\frac{\rho}{\rho_{0}}$ & $T_{\text {model }}[\mathrm{K}]$ \\
\hline 1000 & 47 & 49 & 1.235 & 734 \\
1500 & 79 & 82 & 1.333 & 1385 \\
2000 & 121 & 124 & 1.408 & 2636 \\
2500 & 170 & 172 & 1.493 & 4499 \\
\hline
\end{tabular}


for 2-3 atomic distances (never more); b) kick-out, after which atom, getting to lattice site, remained there forever; c) no changes at all.

One can see that our computer model perfectly fits all experimental data except temperature. It means that there exists another channel of energy dissipation, which is not reduced to chaotic atoms vibrations. As a first 'scientific guess', we assumed that this channel can be a direct use of stress energy for generation of interstitial defects. Let us make elementary evaluations.

Difference between model and real temperatures is about $\Delta T \sim 10^{3} \mathrm{~K}$. Formation energy for interstitial in copper is $2.8 \mathrm{eV}$. If all this extra energy is used for generation of interstitial, their concentration will be $c_{i} \sim k \Delta T / E_{i} \sim 3 \cdot 10^{-2}$. Then effective diffusivity of atoms will be about $D=c_{i} D_{i}=c_{i} D_{i 0} \exp \left(-E_{m}^{i} / k T\right) \sim 3 \cdot 10^{-2} \cdot 10^{-6} \sim 3 \cdot 10^{-8} \mathrm{~m}^{2} / \mathrm{s}$. During period about $10^{-4} \mathrm{~s}$, it will give diffusion zone of about $\sqrt{2 D t} \sim 2 \cdot 10^{-6} \mathrm{~m}$, which appears to be the maximum possible penetration for experiments with shock waves. Yet, authors of the book [58] (and other numerous publications, see, for example, [80-83]) claim that experimentally the penetration depth can reach as much as 100 microns. If they are right, even direct and total consumption of the shock energy by generation of interstitials cannot be the final explanation.

However, at the same time, in our simulations, the nanograins formation has been revealed in the compressed region beyond the SW front prior to decompression wave. Conditions of the shock wave loading were rather unsuitable for detailed analysis of this phenomenon, since the time prior to propagation of decompression wave was too short (time and memory constraints on our computers). To study the lattice reconstruction of the compressed regions in more details, we independently simulated the uniaxially compressed lattice and traced the relaxation processes in it without time limitations of SW propagation. Actually, the nanoscale structure transformations under different ways of deformation have been observed recently [60]. We chose special way of deformation, close to conditions of shock wave loading: we have studied (by MD-simulation) the behaviour of threedimensional, initially single-crystalline $\mathrm{CuNi}$ alloys under uniaxial single and double loading/decompression cycles.

\subsection{Model and Compression Regimes}

Cubic samples with 18-24 elementary f.c.c. cells along each side consisted of 23000-55000 atoms. Periodic boundary conditions had been applied along all three axes $X, Y, Z$. Interatomic interactions had been cut off at the 5 -th coordination shell, so that for each atom in the site 78 interacting neighbours had been taken into account. Interactions have been modelled by using the pairwise Born-Mayer potential for the pure $\mathrm{Cu}$ and the many-body Sutton-Chen potential for the $\mathrm{CuNi}$ alloys 
[84]. Qualitatively the results for both potentials were similar. Time step was taken as 5 fs. Maximal time was $110 \mathrm{ps.} \mathrm{Initial} \mathrm{temperature}$ was $300 \mathrm{~K}$. Before deformation, sample was allowed to relax for $10 \mathrm{ps}$ but with the fixed sample length.

Sample was uniaxially compressed with up to some maximal deformation taken from the results of SW simulation $(35,29,25 \%)$ and stability of lattice without deformation was checked.

Two regimes of compression have been realized: gradual nonuniform compression (generated by gradual motion of sample walls); gradual uniform compression (provided by 'hand-made' uniform rescaling of all interatomic longitudinal coordinates for all atoms between moving walls of sample). At each regime, both single loading or decompression and combination of consecutive loadings/decompresssions have been modelled. In case of consecutive loadings, the second compression has been realized in two ways-along the same axis as the first one, and transversely to it.

In order to quantify the simulation results we used the potential energy distribution $\rho\left(E_{p}\right)$ and distribution $\rho(\Delta)$ of specially designed parameter of non-equiaxiality $\Delta$, which was calculated as logarithm of the ratio of maximal $\left(r_{\max }\right)$ and minimal $\left(r_{\min }\right)$ distances among the 12 nearest neighbours of each atom:

$$
\Delta=\ln \frac{r_{\max }}{r_{\min }} .
$$

Results are qualitatively similar for all simulated alloy concentra-

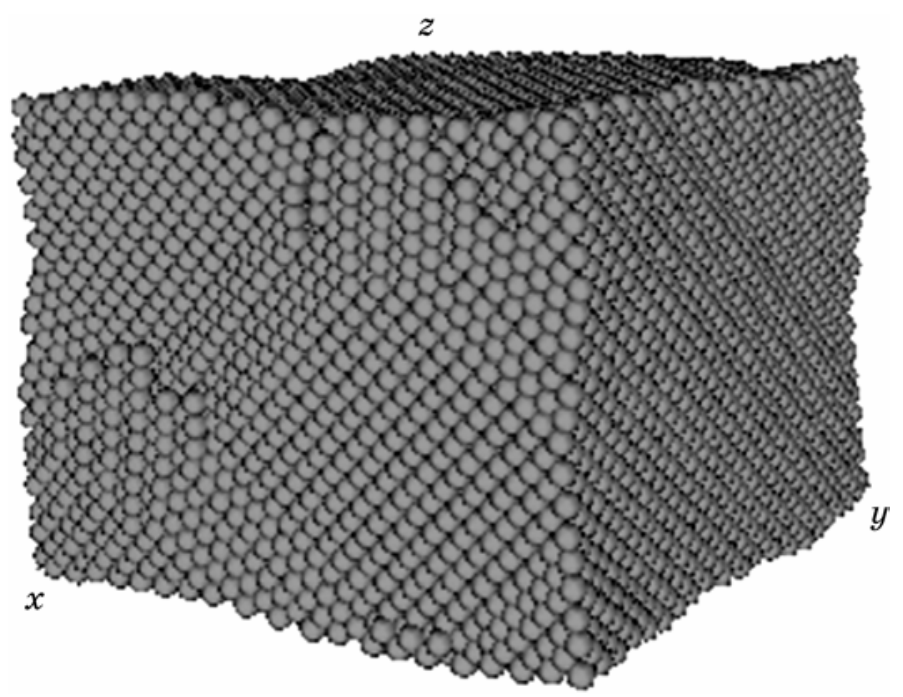

Fig. 9.1. Cubic sample relaxed after uniaxial $25 \%$-deformation, time 35 ps. 
tions, compression regimes, and sample sizes.

Characteristic global picture of lattice reconstruction is shown in Fig. 9.1. The homogeneous regions with different orientationsnanograins-are observed. Boundaries between such regions are visible as well. Thus, initially single-crystalline uniaxially compressed sample transforms into nanocrystalline system. The bulk of each nanograin is significantly more equiaxial comparing with initial state after compression. Simultaneously, atoms at the newly formed grain boundaries have less equiaxial surrounding of nearest neighbours and, respectively, higher potential energy.

For better understanding of the structure transformation, we traced the time evolution of the distribution of atoms over the nonequiaxiality parameter $\Delta$ (Fig. 9.2).

Analysis of dynamics of non-equiaxiality parameter distribution results in following conclusions.

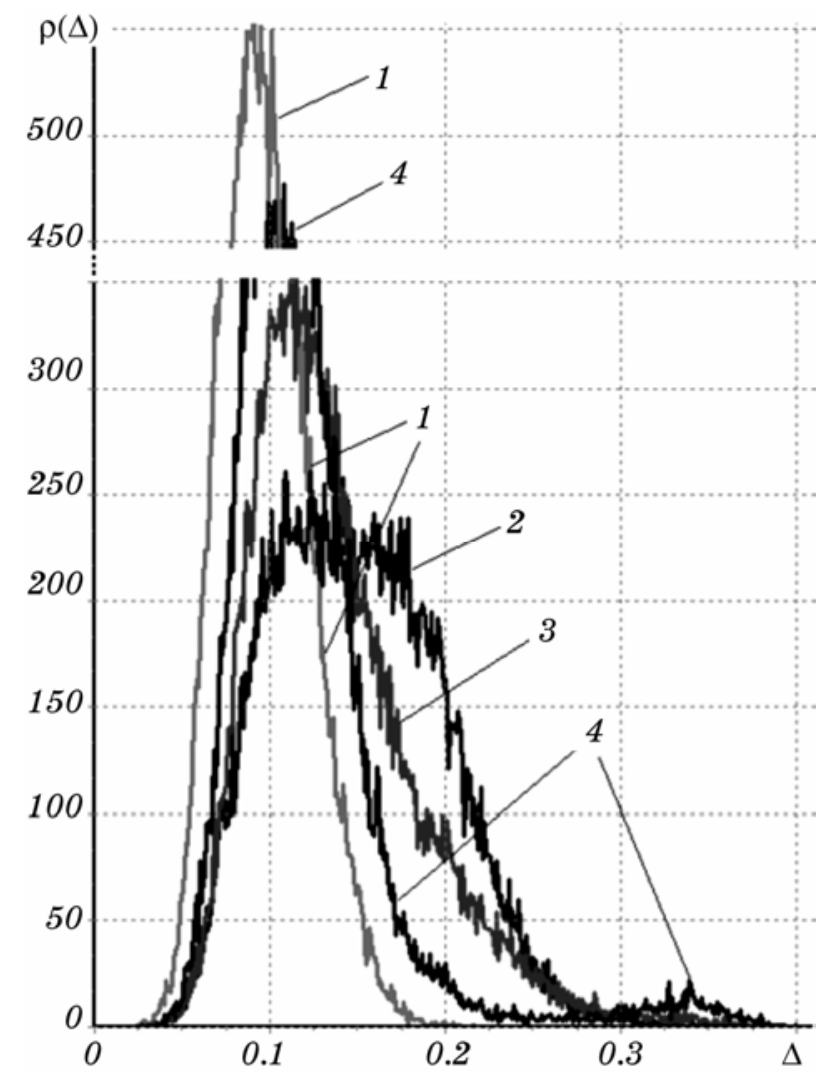

Fig. 9.2. Time evolution of non-equiaxiality parameter distribution after uniaxial $25 \%$-compression (1-before compression, $2-12.5$ ps after loading starts, $3-15 \mathrm{ps}$ after loading starts, $4-20 \mathrm{ps}$ after loading starts). 
1. Initial distribution is obviously a Dirac function, since all atoms are initially in the same conditions.

2. For ideal non-compressed f.c.c. structure, this parameter is equal to zero and due to thermal stochastic vibrations, the distribution of this parameter will be broadened, similar distribution being reached for each initial deformation after about $12.5 \mathrm{ps}$ (plot 1).

3 . For the case of initial compression $25 \%$, the first steps of reconstruction (small shifts and rotations) lead to division of atoms into two classes (plot 2). First (main) class includes atoms, for which the nonequiaxiality of their neighbourhood decreases. Eventually these atoms become part of bulk of the nanograins. Other, smaller, class corresponds to increasing $\Delta$-parameter and includes atoms, which eventually will belong to grain boundaries and their junctions. Formation of this class is almost completed after 15 ps (plot 3), yet, separate peak is

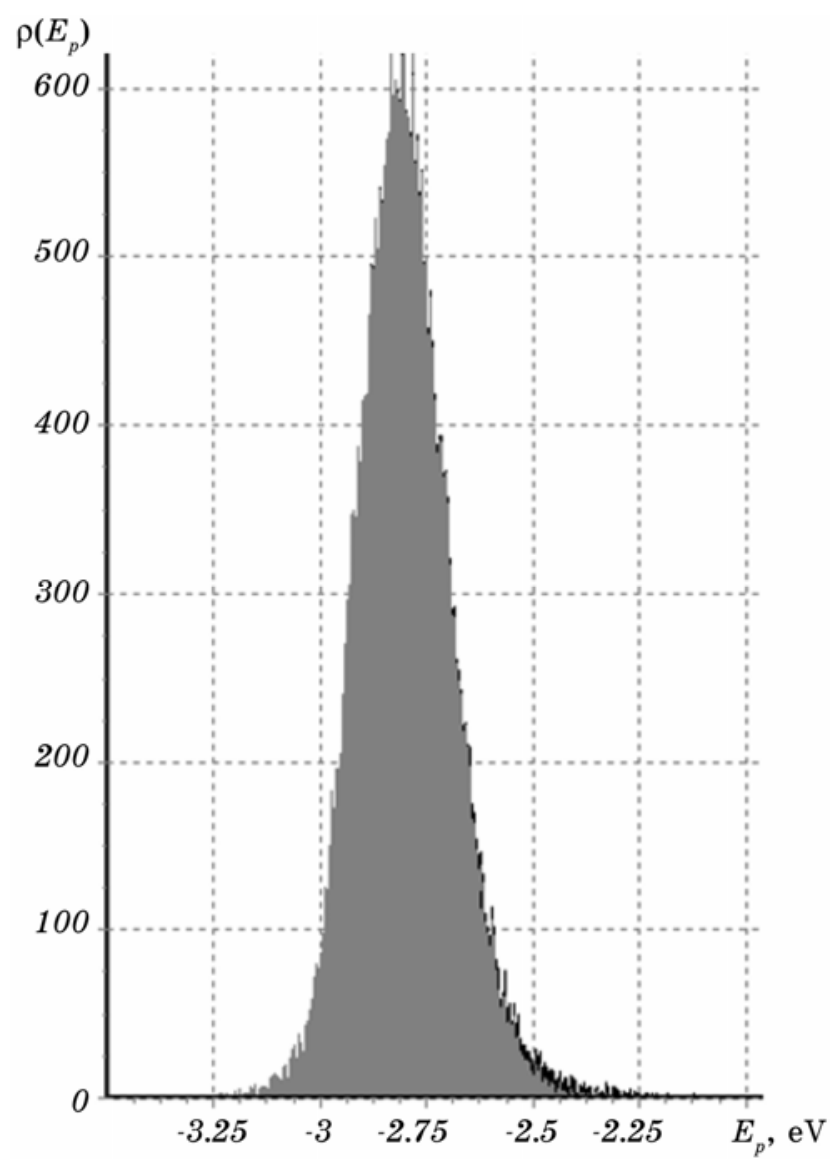

Fig. 9.3. Distribution of potential energy per atom $E_{p}$ (dark region corresponds to the right peak of $\Delta$-distribution). 
formed after much longer period (plot 4).

4. For the case of initial compression $35 \%$, the general picture depends on the way of compression and on the choice of potential. If the compression process is gradual and the interatomic potential is a Born-Mayer one, then the bimodal distribution of $\Delta$-parameter is similar to abovementioned picture. If the deformation is instant, but the interaction is governed by the 'mild' Sutton-Chen potential, we obtain similar picture. Distribution of potential energies is not so sensitive to the processes of reconstruction and does not show two peaks (Fig. 9.3). Atoms belonging to the right peak of $\Delta$-distribution are situated at the right slope of energy distribution (dark region in Fig. 9.3). It means correlation between $\Delta$ and energy.

We determined the spatial distribution of the atoms corresponding to the right peak of $\Delta$-distribution. These atoms with highest degree of parameter $\Delta$ formed parallel one-dimensional chains along the (111)direction. Comparison of Fig. 9.1 and Fig. 9.4 demonstrates that these 'special' atoms find themselves just at grain boundary junctions.

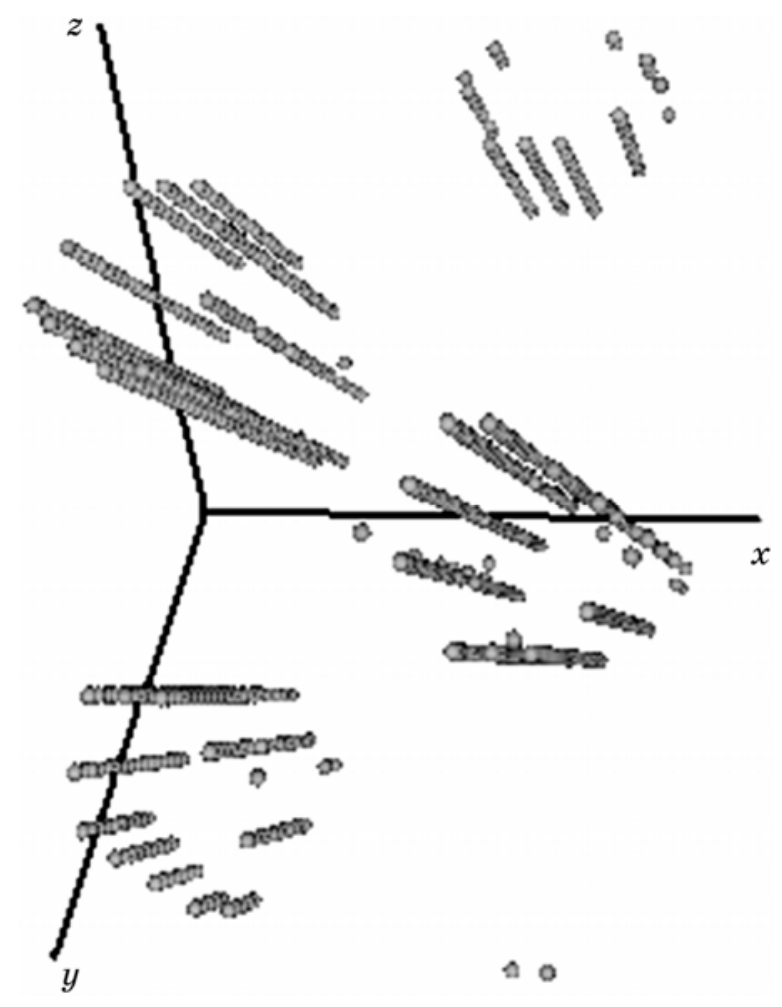

Fig. 9.4. Atoms with highest potential energy and parameter $\Delta$ form parallel one-dimensional chains of atoms along the (111)-direction. Note, that the lattice orientation here is different from lattice orientation in Fig. 9.1. 


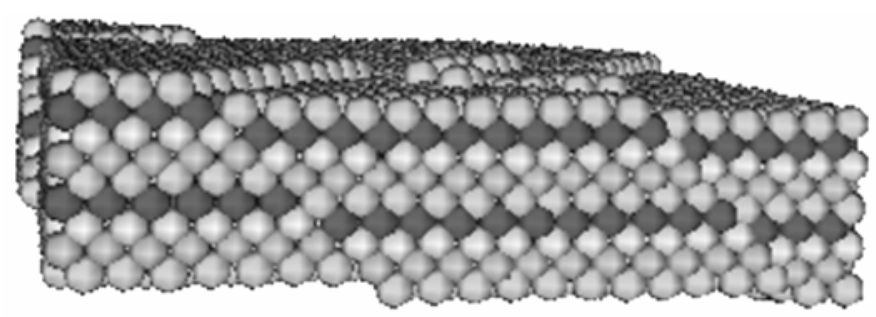

$a$

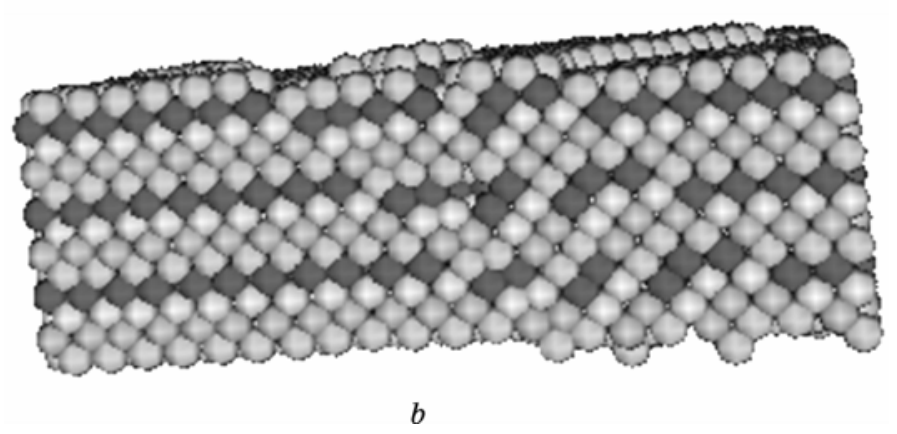

Fig. 9.5. Two fragments of sample after decompression (atoms initially belonged to common plane have been marked with the same colour with period of four planes), time $60 \mathrm{ps}$.

In the certain sense, obtained nanocrystalline structure is 'virtual', since after gradual uniform decompression the number of grains decreases. Yet, the change of short-range order (created by the loading) does not vanish after decompression. Namely, planes, formed from the parts of initially neighbouring planes, remain the common planes after decompression (Fig. 9.5). Thus, the monocrystal becomes a polycrystal, with the number of grains less than in compressed state.

Under second deformation (both in the direction of previous compression and in the perpendicular direction) the results qualitatively coincide with the results of first deformation. Further formation of nanocrystalline structure with larger extent of off-orientation of grains is observed. At the histogram of $\Delta$-parameter, the shift of main peak to the right and its broadening after second compression is observed. It means the decrease of 'bulk fraction', i.e. the higher degree of grain crushing.

It can be assumed that during the pulse loading the ensemble of microwaves of loading/decompression propagates. Their superposition can lead to the intermixing with creation of nanostructure and large relative shifts of nanograins. Because of multiple propagations of shock waves and decompression waves, the substantial transport of matter (anomalous mass transfer) is possible. Thus, we came back to 
the initial Ruoff's idea [61] of mechanical diffusion, but now at nanolevel. As for abovementioned arguments about the immobility of molecules, we can argue that in salt the tracers are incorporated not just in molecules, but also in crystalline grains of salt, which, of course, are larger than the size of nanograins, and therefore cannot be just intermixed.

\section{FUTURE DEVELOPMENTS}

So far, we have some set of thermodynamic and kinetic models for description of phase nucleation, growth and competition. Yet, the picture is still incomplete. Here we will indicate only some of future developments.

1. Models of nucleation in the concentration gradient have been developed so far mainly for the case, when parent metastable phase has broad concentration range. In many real cases it is not so. Recently [85] a new possible description of nucleation and sequential phase growth in thin films was proposed in the frame of 'nucleation in concentration gradient' approach. If at least one phase with narrow homogeneity range is already growing, the sharp gradient of chemical potential in it strongly influences the nucleation barrier for next phase to appear. Gradient input to size dependence of Gibbs energy change appears to be proportional to the fourth power of size instead of fifth power in previous models. As a result, thermodynamic suppression (in addition to kinetic suppression) may be effective with suppressing phases thickness of tens or even hundreds nanometres. Thus, 'gradient term effect' may well lead to the total absence of suppressed phases prior to consumption of thin film by suppressing growing phase.

2. Almost simultaneous multiple nucleation, leading to formation of bulk nanocrystalline alloys, presents a new challenge for materials science-ripening just after nucleation, without intermediate growth stage, and with large volume fraction of coarsening phase. It is now well established that LSW (Lifshitz-Slyozov-Wagner) theory of ripening does not work in this case. LSW theory is correct for negligibly small volume fractions $f$, and it can be modified in terms of perturbation theory for cases of still very small $f$. Yet, there exists a critical value of volume fraction, above which LSW is not to be used even with perturbation, because the diffusive screening length is reduced to inter-particle distance. For these 'large' volume fractions (usually occurring in most experiments), each precipitate can effectively exchange atoms only with nearest neighbours. For this case recently, a new approach has been proposed [86]-'ripening in the normalized space' (invented initially for description of normal grain growth [87]), making possible to take into account a cooperative character of ripening with only the nearest-neighbour diffusive interactions. The pre- 
dicted particle size distribution has no cut-off. It is broader and lower, and it has small positive skewness (instead of a large negative one in LSW). We hope that this approach can be helpful also for nanoalloys.

3. One more important problem is a flux-driven coarsening in nanosystems. In open system, kinetics of coarsening may well be controlled not just by curvature of interfaces, but instead by external fluxes. This idea has been used recently for interpreting the flux-driven ripening during reactive diffusion [88] and the flux-driven grain growth during thin film deposition [89].

4. There is still some problem with using proper diffusivities $D$ for description of intediffusion in nanosystems. Most often $D$ is treated as an interdiffusion coefficient, determined by the well-known Darken's expression [90] with Manning's corrections (due to vacancy wind effect) [91]. Yet, Darken's approach is based on the account of Kirkendall shift of lattice planes. This shift proceeds due to vacancy flux, resulting from difference of intrinsic diffusivities of species: $D_{A} \neq D_{B}$. Mechanism of Kirkendall shift is the dislocation climbing in the processes of vacancy generation and annihilation-building up of extraplanes in one part of the diffusion couple (containing the slower diffusing species) and dismantling of planes in another part (containing the faster species). In nanosystems, the characteristic size may be less than the mean free path of vacancy migration between sources and sinks. As was shown, for example in [4, 92], in this case Darken's analysis is not applicable because the redistribution of species in mesoscopic regions between vacancy sources and sinks is governed not by Darken's interdiffusivity

$$
D_{D A R K E N}=\left(C_{B} D_{A}^{*}+C_{A} D_{B}^{*}\right) \cdot \frac{C_{A} C_{B}}{k T} g^{\prime \prime},
$$

but instead by effective diffusivity

$$
D_{e f f}=\frac{D_{A} D_{B}}{C_{A} D_{A}+C_{B} D_{B}} \cdot \frac{C_{A} C_{B}}{k T} g^{\prime \prime}
$$

(known, for example, from diffusion theory of ionic crystals). The main difference is that Darken's diffusivity in concentrated alloys is determined mainly by the fast species, but the effective diffusivity is determined by the slow species.

\section{ACKNOWLEDGEMENTS}

Present work is supported by International Association for Promotion of Cooperation with Scientists from New Independent States of the former Soviet Union (INTAS grant \# 784, young NIS Scientist Fellowships Programme 2003-INTAS Ref. No. 03-55-1169), in part by 
CRDF (grant \#UE1-2523-CK-03) and Ministry of Education and Science of Ukraine. Additionally to colleagues, acknowledged in Introduction, authors are grateful to F. d'Heurle, P. Gas, G. Martin, and J. Schmelzer for helpful discussions of nucleation/growth issues.

\section{REFERENCES}

1. Ya. E. Geguzin, Diffusionnaya Zona (Diffusion Zone) (Moscow: Nauka: 1981) (in Russian).

2. C. P. Gurov, B. A. Kartashkin, and Yu. E. Ugaste, Vzaimnaya Diffusiya $v$ Mnogofaznykh Metallicheskikh Sistemakh (Interdiffusion in Multiphase Metallic Systems) (Moscow: Nauka: 1981) (in Russian).

3. A. V. Nazarov and K. P. Gurov, Fiz. Met. Metalloved., 38, No. 4: 698 (1975) (in Russian).

4. K. P. Gurov and A. M. Gusak, Fiz. Met. Metalloved., 59, No. 6: 1062 (1985) (in Russian).

5. F. Hartung and G. Schmitz, Phys. Rev. B, 64: 245418 (2001).

6. I. Kaur and W. Gust, Fundamentals of Grain and Interphase Boundary Diffusion (Stuttgart: Ziegler Press: 1989); idem (Moscow: Mashinostroenie 1991) (Russian translation).

7. F. M. d'Heurle, J. Materials Research, 3: 167 (1988).

8. B. Ya. Pines, Essays on Metal Physics (Kharkov: University Publ.: 1961) (in Russian).

9. J. Philibert, Defect and Diffusion Forum, 66-69: 995 (1989).

10. V. I. Dybkov, J. Materials Science, 21, No. 9: 3078 (1986); ibidem, 22, No. 12: 4233 (1986).

11. U. Goesele and K. N. Tu, J. Appl. Phys., 53: 3552 (1982).

12. W. L. Johnson, J. Mater. Sci., 30: 81 (1986).

13. V. Vieregge and Chr. Herzig, J. Nucl. Mater., 175: 29 (1990).

14. C. Michaelsen, K. Barmak, and T. P. Weihs, J. Phys. D: Appl. Phys., No. 30: 3167 (1997).

15. E. Emeric, P. Gas, G. Clugnet, and C. Bergman, Microelectronic Engineering, 50: 285 (2000).

16. E. Emeric, Etude des Reactions a l'Etat Solide dans des Multicouches Al/Co (Thesis of Disser. for Ph.D.) (Marseille: 1998) (in French).

17. T. Jeske and G. Schmitz, Scripta Mat., 45: 555 (2001).

18. A. Inoue, T. Zhang, and T. Masumoto, Mater. Trans. JIM, 31: 425 (1990).

19. A. Paker and W. L. Johnson, Appl. Phys. Lett., 63: 2342 (1993).

20. M. Calin and U. Koster, Mater. Sci. Forum, 269-272: 749 (1997).

21. K. F. Kelton, Phil. Mag. Lett., 77, No 6: 337 (1998).

22. A. M. Gusak and K. P. Gurov, Fiz. Met. Metalloved., 53, No. 5: 842 (1982) (in Russian).

23. A. M. Gusak and K. P. Gurov, Izv. AN SSSR. Metal., No. 1: 163 (1990) (in Russian).

24. A. M. Gusak and K. P. Gurov, Fiz. Met. Metalloved., 53, No. 5: 848 (1982) (in Russian).

25. A. M. Gusak and Yu. A. Lyashenko, Fiz. Khim. Obrabotki Mater., No. 5: 140 (1993) (in Russian). 
26. A. M. Gusak, Poroshkovaya Metallurgiya, No. 3: 39 (1989) (in Russian).

27. A. M. Gusak and G. V. Lucenko, Acta Mater., 46, No. 10: 3343 (1998).

28. K. N. Tu, A. M. Gusak, and M. Li, J. Appl. Phys., 93, No. 3: 1335 (2003).

29. K. Tu (private communication).

30. A. M. Gusak, Ukr. Fiz. Zh., No. 5: 725 (1990) (in Russian)

31. A. M. Gusak and K. P. Gurov, Solid State Phenomena, 23\&24: 117 (1992).

32. P. J. Desré and A. P. Yavari, Phys. Rev. Lett., 64: 1553 (1990).

33. P. J. Desré, Acta Metal., 39, No. 10: 2309 (1991).

34. A. M. Gusak and A. V. Nazarov, Metallofizika, 12, No. 2: 48 (1990) (in Russian).

35. A. M. Gusak and A. V. Nazarov, J. Phys. Condens. Matter, 4: 4753 (1992).

36. F. Hodaj and P. J. Desré, Acta Mater., 44, No. 11: 4485 (1996).

37. F. Hodaj, A. M. Gusak, and P. J. Desré, Phil. Mag. A, 77: 1471 (1998).

38. A. M. Gusak, F. Hodaj, and A. O. Bogatyrev, J. Phys.: Condens. Matter, 13, No. 12: 2767 (2001).

39. A. M. Gusak, O. V. Dubiy, S. V. Kornienko, Ukr. Fiz. Zh., 36, No. 2: 286 (1991) (in Russian).

40. A. M. Gusak and A. O. Bogatyrev, Metallofizika, 16, No. 9: 28 (1994) (in Russian).

41. A. M. Gusak, Yu. A. Lyashenko, and A. O. Bogatyrev, Solid-Solid Phase Transformation (Eds. W. C. Johnson et al.) (1994), p. 1171.

42. A. M. Gusak, Yu. A. Lyashenko, and A. O. Bogatyryev, Defect and Diffusion Forum, 129-130: 95 (1996).

43. A. O. Bogatyrev and A. M. Gusak, Metallofiz. Noveishie Tekhnol., 18: 897 (2000).

44. A. M. Gusak, Yu. A. Lyashenko, and S. V. Kornienko, Functional Materials, 6, No 2: 242 (1999).

45. A. O. Bogatyrev, F. Hodaj, and A. M. Gusak, Bull. of Cherkasy State University. Physics, 37-38: 89 (2001-2002).

46. L. Farkas, Z. Phys. Chem., 125: 239 (1927).

47. Y. B. Zeldovich, Acta Physicochim., 18: 1 (1943).

48. G. Lucadamo, K. Barmak, S. Hyun, C. Cabral, Jr., and C. Lavoie, Materials Letters, 39: 268 (1999).

49. A. M. Gusak and A. S. Shirinyan, Metallofiz. Noveishie Tekhnol., 20, No. 6: 40 (1998) (in Russian).

50. A. S. Shirinyan and A. M. Gusak, Ukr. Fiz. Zh., 44, No. 7: 883 (1999) (in Russian).

51. A. I. Rusanov, Fazovyye Ravnovesia i Poverkhnostnyye Yavleniya (Phase Equilibria and Surface Phenomena) (Leningrad: Chemistry: 1967) (in Russian).

52. H. Ulbricht, J. Schmelzer, R. Mahnke, and F. Schweitzer, Thermodynamics of Finite Systems and Kinetics of First-Order Phase Transitions (Leipzig: BSB Teubner: 1988).

53. A. S. Shirinyan, A. M. Gusak, and P. J. Desré, J. of Metastable and Nanocrystalline Materials, 7: 17 (2000) (http:/www.scientific.net/jmnm).

54. L. N. Larikov and O. A. Shmatko, Cellular Decomposition of Oversaturated Solid Solutions (Kiev: Naukova Dumka: 1976) (in Russian).

55. J. W. Cahn, Acta Metall., 9: 795 (1961).

56. A. G. Khachaturyan, The Theory of Phase Transformations and the Struc- 
ture of Solid Solutions (Moscow: Nauka: 1974) (in Russian).

57. Yu. A. Lyashenko, Technical Physics Letters, 30, No 2: 109 (2004) (translated from Pis'ma $v$ Zhurnal Teknicheskoi Fiziki, 30, No 3: 54-61 (2004)).

58. D. S. Gertzricken, V. F. Mazanko, and V. M. Fal'chenko, Pulse Processing and Mass-Transfer in Metals at Low Temperatures (Kiev: Naukova Dumka: 1991) (in Russian)

59. D. S. Gertzricken, T. V. Kolenova, and A. M. Gusak, Defect and Diffusion Forum, 194-199: 1469 (2001).

60. M. A. Meyers, F. Gregori, B.K. Kad, M. S. Schneider et al., Acta Materialia, 51: 1211 (2003).

61. A. L. Ruoff, J. Appl. Phys., 38, No. 10: 3999 (1967).

62. A. M. Gusak and A. O. Kovalchuk, Phys. Rev. B, 58, No. 5: 2551 (1998).

63. M. Athenes, P. Bellon, and G. Martin, Phil. Mag. A, 76, No. 3: 565 (1997).

64. A. M. Gusak, A. O. Bogatyrev, and A. O. Kovalchuk, Defect and Diffusion Forum, 194-199: 1625 (2000).

65. F. J. J. van Loo, Prog. Solid State Chem., 20: 47 (1990).

66. A. M. Gusak and M. V. Yarmolenko, J. of Applied Physics, 73: 4881 (1993).

67. G. Lucadamo, K. Barmak, D. T. Carpenter, C. Lavoie et al., Mat. Res. Symp. Proc., 562: 159 (1999).

68. L. Klinger, Y. Brechet, and G. Purdy, Acta mater., 46: No. 8: 2617 (1998).

69. G. Lucenko and A. Gusak, Bull. of Cherkasy State University. Physics, 3738: 145 (2001-2002).

70. G. Lucenko and A. Gusak, Microelectronic Engineering, 70: 529 (2003).

71. J. W. Cahn and J. E. Hilliard, J. Chem. Phys., 31: 688 (1959).

72. A. S. Shirinyan and A. M. Gusak, Phil. Mag. A, 84, No. 6: 579 (2004).

73. A. S. Shirinyan, Proc. of 15-th Intern. Symp 'Thin Films in Optics and Electronics' (Eds. A. F. Belyanin, V. I. Lapshin, I. E. Protsenko, and

V. M. Shulayev) (Kharkov: 2003) (in Russian).

74. J. W. Cahn, Acta. Metall., 7: 18 (1959).

75. G. R. Speich, Trans. Metall. Soc. AIME, 242: 1359 (1968).

76. H. Ziegler, Progress in Solid Mechanics (Eds. I. N. Sneddon et al.) (Amsterdam: North-Holland: 1963), vol. 4, chap. 2, p. 93.

77. S. R. De Groot and P. Mazur, Non-Equilibrium Thermodynamics (Amsterdam: North-Holland: 1962); idem (Moscow: Mir: 1964) (Russian translation).

78. I. Gyarmati, Non-Equilibrium Thermodynamics (Berlin: Springer-Verlag: 1970); idem (Moscow: Mir: 1974) (Russian translation).

79. J. S. Kirkaldy and D. J. Young, Diffusion in the Condensed State (London: The Institute of Metals: 1987).

80. L. N. Larikov, V. M. Fal'chenko, V. F. Mazanko et al., Avtomaticheskaya Svarka, 5: 19 (1974) (in Russian).

81. L. N. Larikov, V. M. Fal'chenko, V. F. Mazanko et al., Dokl. AN Ukr. SSR, 2: 167 (1975).

82. M. E. Gourevich, L. N. Larikov, A. E. Pogorelov et al., Phys. status solidi A, 76: 479 (1983).

83. D. S. Gertzricken, V. F. Mazanko, V. M. Tyshkevich, and V. M. Fal'chenko, Mass-Transfer in Metals at Low Temperatures under External Actions (Kyyiv: 1999) (Prepr./N.A.S. of the Ukraine. Inst. for Metal Physics) (in Russian).

84. Y. Qi, T. Gagin, Y. Kimura, and W. A. Goddard III, Phys. Rev. B, 59: 3527 
502 A. M. Gusak, A. O. Bogatyrev, A. O. Kovalchuk, S. V. Kornienko et al.

(1999).

85. F. Hodaj and A. Gusak (submitted to Acta Mater.)

86. A. Gusak and K. N. Tu. (submitted to Acta Mater.)

87. A. Gusak and K. N. Tu, Acta Mater., 51: 3895 (2003).

88. A. M. Gusak and K. N. Tu, Phys. Rev. B, 66: 115403 (2002).

89. A. M. Gusak, K. N. Tu, and I. Sobchenko, Phys. Rev. B, 67: 245408 (2003).

90. L. S. Darken, Trans. AIME, 175: 184 (1948).

91. J. R. Manning, Acta Metall., 15: 817 (1967).

92. A. M. Gusak and K. P. Gurov, Solid-Solid Phase Transformation (Eds. W. C. Johnson et al.) (1994), p. 1133. 